\title{
Biochemical Analysis of Slam - a polarity protein during early Drosophila embryogenesis
}

\author{
Dissertation
}

for the award of the degree "Doctor rerum naturalium (Dr.rer.nat.)" in the GGNB program "Biomolecules: Structure-Function-Dynamics" at the Georg-August-Universität Göttingen

submitted by

Stephanie Häusser (birth name Gröning)

born in Crivitz, Germany

Göttingen, July 2021 


\section{Members of the Thesis Committee}

Prof. Jörg Großhans (Supervisor, reviewer)

Developmental Biochemistry, Medical School of Göttingen

Prof. Heike Krebber (Reviewer)

Molecular Genetics, University of Göttingen

Prof. Ralf Ficner

Molecular Structural Biology, University of Göttingen

\section{Additional Members of the Examination Board}

Prof. Gregor Bucher

Developmental Biology, University of Göttingen

\section{Prof. Gerd Vorbrüggen}

Molecular Developmental Biology, Max Planck Institute for Biophysical Chemistry

\section{Prof. Andreas Janshoff}

Physical Chemistry, University of Göttingen 


\section{Publications}

Yan S, Acharya S, Gröning S, Großhans J. Slam protein dictates subcellular localization and translation of its own mRNA. PLoS Biol. 2017 Dec 4;15(12):e2003315. doi:

10.1371/journal.pbio.2003315. PMID: 29206227; PMCID: PMC5730382.

Winkler F, Kriebel M, Clever M, Gröning S, Großhans J. Essential Function of the Serine Hydroxymethyl Transferase (SHMT) Gene During Rapid Syncytial Cell Cycles in Drosophila. G3 (Bethesda). 2017 Jul 5;7(7):2305-2314. doi: 10.1534/g3.117.043133. PMID: 28515048; PMCID: PMC5499137. 


\section{Acknowledgements}

First, I would like to express my gratitude to Prof. Dr. Jörg Großhans who gave me the opportunity for my doctoral studies. His guidance, patience, and encouragement during the time of research and writing was elementary for the success of this thesis.

I would like to thank my thesis committee members Prof. Dr. Ralf Ficner and Prof. Dr. Heike Krebber. Thank you for investing the time and your helpful comments and support. The different perspective on my work was most helpful.

I would like to acknowledge my colleagues for their help and insightful discussions. My special thanks go to my immediate team Dr. Sreemukta Acharya and Dr. Shuling Yan, who would answer any Slam-related question and did the groundwork to this study. I truly missed you in the lab Mukta! I thank Dr. Zhiyi Lv, Dr. Anja Schmidt, Dr. Maria Kriebel and Dr. Deqing Kong for being obliging colleagues, who would always offer advice and help. Thank you to Johannes Sattmann for assistance and Manuela Manafas for the administrative support and the nice "floor chats"!

I truly appreciate my graduate school for their constant support and commitment and GAUSS for providing a funding for the last months of my work. I am thankful for the SFB86o, which funded most of my work.

My deepest appreciation goes to my parents, grandparents (the ones in heaven and on earth) and sisters without whom I would never have had the opportunities that lead to this work. I am thankful for my family and friends, who were a welcome change to the scientific world and were constant support during the difficult times that lie behind us all.

I am indebted to my husband, Arvid Häusser, for the support, especially during the last weeks of writing. Thank you for giving me insights into creative cloud programs, for superb coffee and constant appreciation. I want to thank my children, who changed my view on life and priorities and gave research a different purpose.

All you proteins out there: thank you for being! Each of you so unique and challenging to understand. Life would not only be impossible but also boring without you. I always did, and always will love to work with you! 


\section{Table of content}

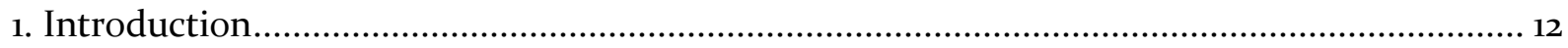

1.1 Early development of Drosophila melanogaster ................................................................. 13

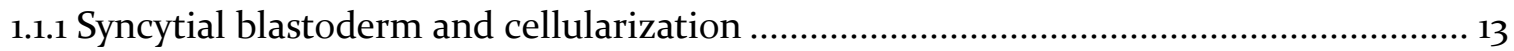

1.1.2 Metaphase furrows and actin caps during syncytial cycles............................................. 14

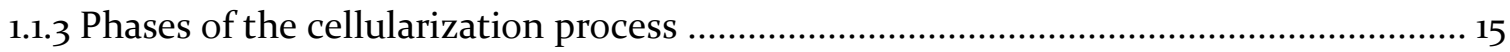

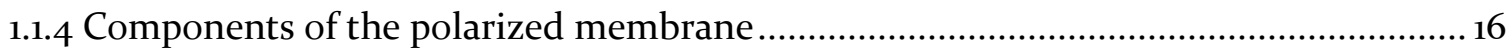

1.1.5 Slam recruits Myosin II to the furrow canal .................................................................. 18

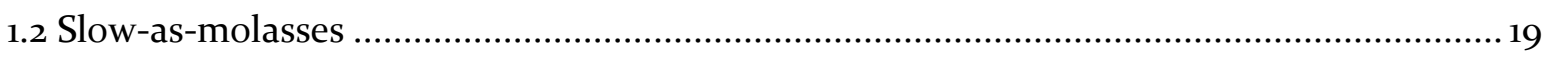

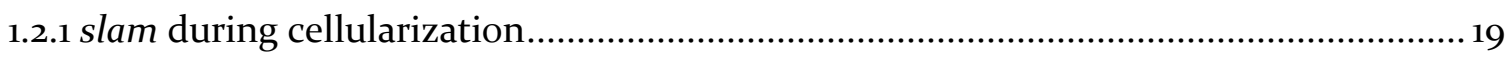

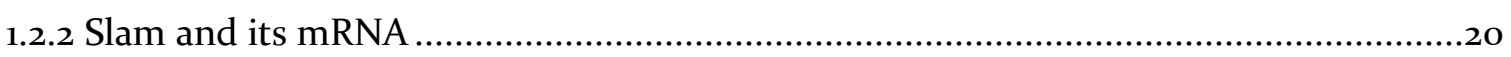

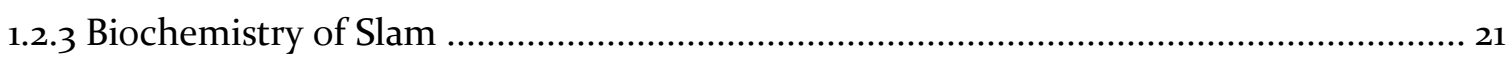

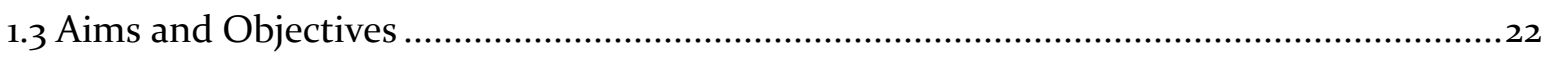

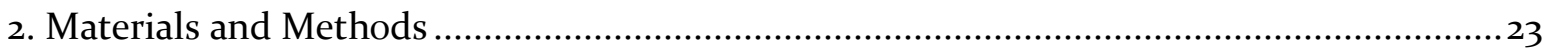

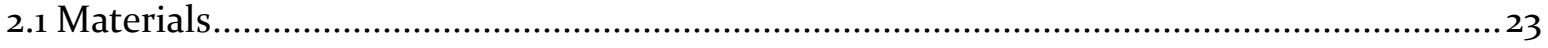

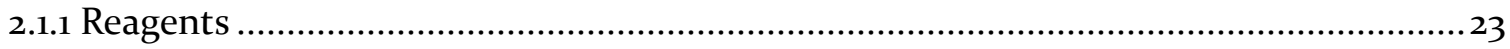

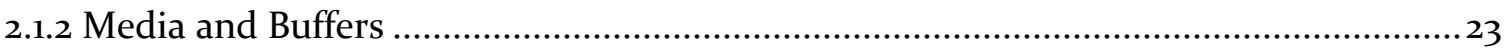

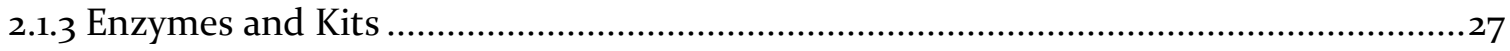

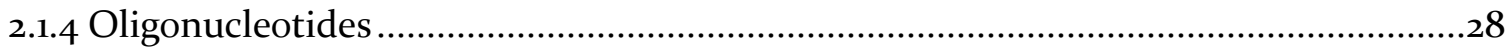

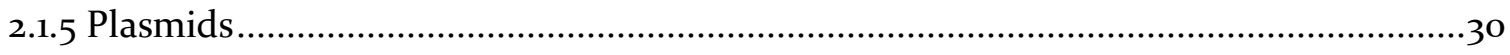

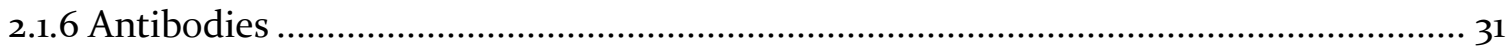

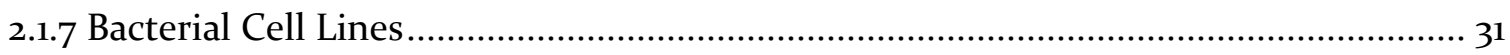

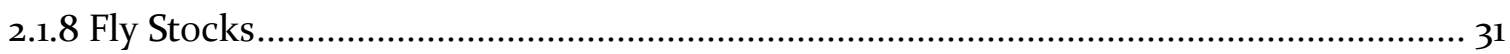

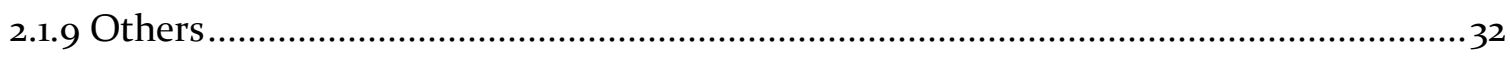

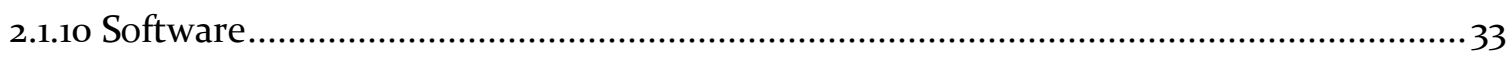

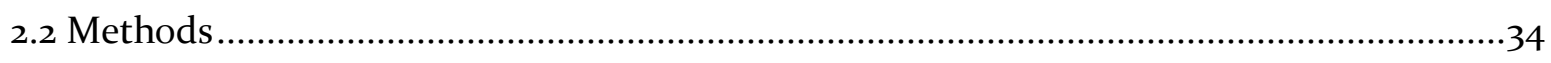

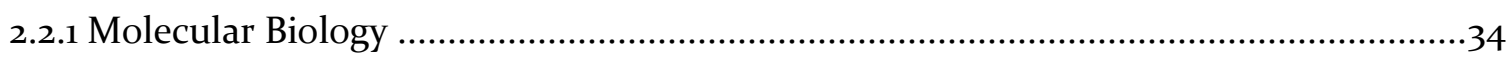

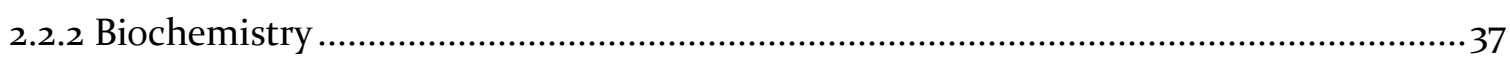

2.2.3 Developmental Biology ….............................................................................. 41

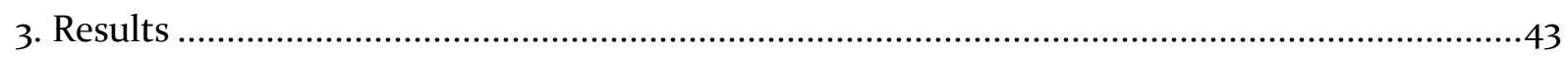

3.1 Expression and purification of recombinant protein fragments of Slam ..........................43

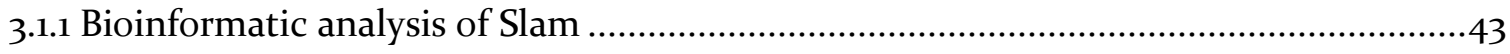

3.1.2 Purification of recombinant Slam fragments ...........................................................47

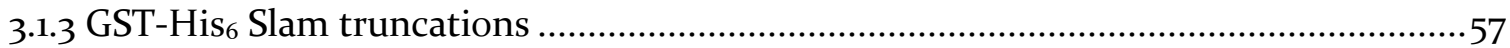




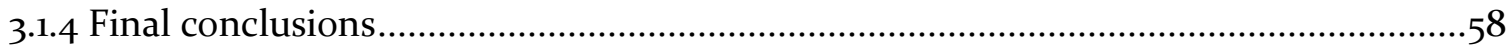

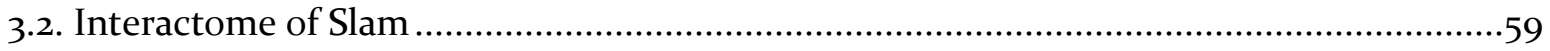

3.2.1 Putative interactors in data bases and previous reports...........................................59

3.2.2 Immunoprecipitation-mass spectrometry (IP-MS) based identification of Slam

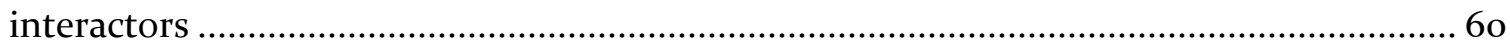

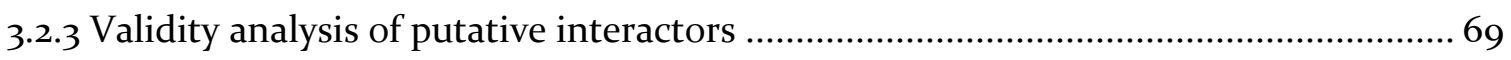

3.3 Post-translational modifications and stability of Slam.......................................................78

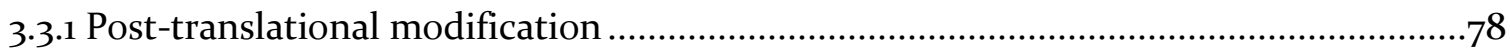

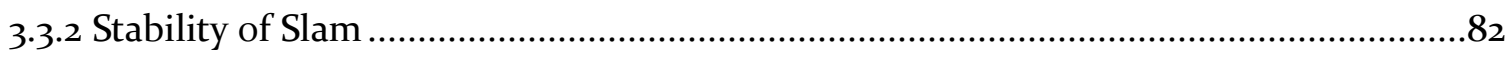

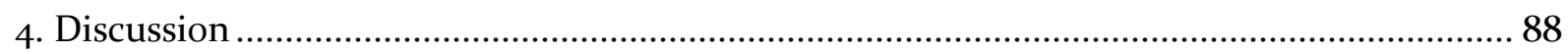

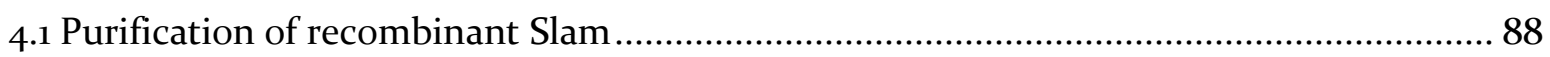

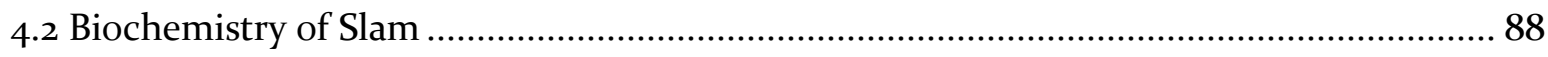

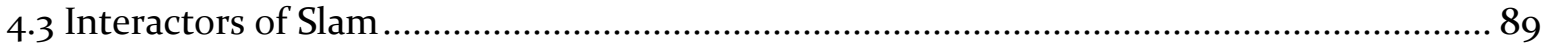

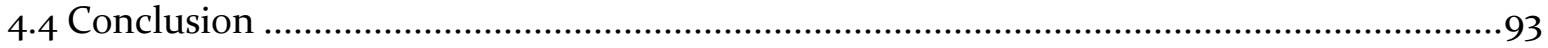




\section{List of Figures}

Figure 1 Simplified depiction of early Drosophila development.

Figure 2 Cytoskeletal rearrangement during syncytial cycles

Figure 3 Phases during cellularization

16

Figure ${ }_{4}$ Components of the metaphase furrow and the early cellularization furrow. 18

Figure 5 Assembly of Western Blot 38

Figure 6 PageRuler ${ }^{\mathrm{TM}}$ Plus Prestained Protein Ladder (fishersci.de) .38

Figure 7 Sequence Plot of Slam (LD36405p)

Figure 8 Sequence Plot of Slam (LD36405p)

Figure 9 Slam disorder prediction using DISOPRED3.

Figure 10 Amino acid sequence of Slam 46

Figure 11 Expression and solubility of recombinantly produced Slam protein.

Figure 12 Expression and solubility of recombinantly produced truncated Slam. 48

Figure 13 GST-Slam ${ }^{24-527}$ solubility is increased with changing growth medium for the expression host. 48

Figure 14 Purification of GST-Slam ${ }^{24-527}$. 48

Figure 15 Overview of the multistep purification procedure of GST- Slam ${ }^{1069-1196}$...... 49

Figure 16 Capturing step of GST- Slam ${ }^{1069-1196}$ purification 50 
Figure 17 Reapplication of protease-treated protein mixture to the GSTrap $4 \mathrm{~B}$ to separate tag and Slam ${ }^{1069-1196}$

Figure 18 Anion-exchange chromatography of Slam ${ }^{1069-1196}$

Figure 19 Last step of the multi-step purification of Slam ${ }^{1069-1196}$

Figure 20 Analysis of GST- Slam ${ }^{1069-1196}$ and GST- Slam ${ }^{1069-1196}-\mathrm{His}_{6}$ solubility.........

Figure 21 Solubility of GST-Slam ${ }^{1069-1196}-\mathrm{His}_{6}$

Figure 22 GST-Slam ${ }^{1069-1196}$-His 6 two-step purification plan.

Figure 23 Purification of GST- Slam ${ }^{1069-1196}-\mathrm{His}_{6}$ .56

Figure 24 Purification of GST- Slam ${ }^{1069-1196}-\mathrm{His}_{6}$

Figure 25 Slam truncations for a solubility screen. .58

Figure 26 Slam is associated to membranes. 61

Figure 27 GFPSlam pull-down assay for mass spectrometric analysis of putative Slam binding partners.

Figure 28 Slam double band. 78

Figure 29 Putative phosphorylation of Slam. 79

Figure 30 Analytics repetition of phosphorylation assay samples. 79

Figure 31 Quantitative profile of Slam during the first hours of Drosophila deleopment. 81

Figure 32 Slam degrades gradually in an embryonic lysate.. 82 
Figure 33 Chromatogram (A) and analysis (B) of a Superdex 200 gel-filtration of Drosophila (WT) embryonic extract (1.5-2.5 h) 83

Figure 34 Slam is almost undetectable in samples after RNase treatment. 84

Figure 35 Titration of RNase to the lysate of cellularizing fly embryos. 86

Figure 36 Degradation of Slam in lysate is faster in the presence of additional RNase. 87

Figure 37 Schematic overview of hypothetical models presenting functions of adaptor proteins .92

Figure 38 Interactions between a selection of identified proteins coprecipitated with Slam referred to flybase.org (physical interactions). 


\section{Abbreviations}

${ }^{\circ} \mathrm{C}$

$\mu$

aa

AAJ

BAJ

bp

cDNA

$\mathrm{ddH}_{2} \mathrm{O}$

DNA

DTT

E. coli

EDTA

FC

FE

g

GFP

GST

h

IPTG

$\mathrm{kb}$

$\mathrm{kDa}$

m

$\min$

mRNA

$\mathrm{Nr}$

PCR

PMSF

RNA

RNP

rpm

RT

SC degree Celsius

micro-

amino acid(s)

apical adherens junctions

basal adherens junctions

base pairs

complementary DNA

double deionised water

deoxyribonucleic acid

1,4-dithiothreitol

Escherichia coli

ethylenediaminetetraacetic acid

furrow canal

fold enrichment

$\operatorname{gram}(\mathrm{s})$

green fluorescent protein

Glutathione-S-transferase

hour(s)

isopropyl- $\beta$ - $\mathrm{D}$-thiogalactopyranoside

kilobases

kiloDalton

litre(s)

milli-

minute(s)

messenger RNA

number

polymerase chain reaction

Phenylmethylsulfonylfluorid

ribonucleic acid

ribonucleoprotein

revolutions per minute

room temperature

normalized total spectral counts 
SDS

SDS-PAGE

Slam

SS

Tris

WT sodiumdodecylsulphate

SDS-polyacrylamide gel electrophoresis

slow as molasses

significance

tris(hydroxymethyl)aminomethnehydrochloride

wild type 


\section{Summary}

Slow as molasses (slam) is a gene essential for embryonic development of Drosophila. Slam is necessary for the formation and ingression of the plasma membrane and its polarisation, i.e. the formation of the basal cortical domain. Later, Slam controls the directed migration of primordial germ cells. Slam may organise a platform for Rho signalling and membrane trafficking. Slam displays the striking feature that Slam protein and slam mRNA colocalize at the basal domain and form a complex which can be isolated by immunoprecipitation. The interaction of the protein and the mRNA at the basal domain of the plasma membrane is important for the mRNA localization and its efficient translation.

However, little is known about the molecular mechanisms by which Slam fulfils its tasks. Slam encodes an unconserved protein of 1196 amino acid residues with no obvious domains and with large regions predicted to be structurally disordered.

This study aims to further investigate the biochemistry and molecular interactors of Slam to advance the understanding of membrane formation and polarisation. Firstly, a series of Slam truncations were constructed and expressed in E. coli to identify Slam fragments which may be suitable for crystallographic studies in the future. Investigating Slam's structure will give fundamental insights into its nature and way of function. A protocol for the purification of the C-terminal 129 aa region of Slam was developed and optimised to generate high-purity submilligram amounts of the fragment.

Secondly, the proteome of Slam interactors was isolated and identified by mass spectrometry. To this end, a multistep immunoprecipitation protocol was developed. Previously known interactors were confirmed. Strikingly, the interactors fall into functional classes. Multiple components of the actin cytoskeleton were isolated, such as components of the Arp2/3 complex, alpha-actinin and non-muscle Myosin II. Furthermore, components related to the cell cortex and membrane trafficking were found, including Cindr, Restin homolog (clip19o) and Jaguar (Myosin 95F). The list of interactors will serve as a starting point for future studies resolving the role of Slam for membrane formation and its interaction with the actin cortex. 


\section{Introduction}

\subsection{Early development of Drosophila melanogaster}

\subsubsection{Syncytial blastoderm and cellularization}

As in most other species of the animal kingdom, the early development of Drosophila melanogaster starts with a fertilized egg. Peculiar about insects, like flies, however is that they continue their embryogenesis in a coenocytic blastoderm, also called the syncytium. (Willmer 1990). After fertilization (fig. 1A) the zygotic nuclei undergo 13 rounds of mitotic divisions omitting cytokinesis, leading to a multinucleate cell: the syncytial blastoderm. The first 9 rounds of mitotic divisions take place in the interior of the single cell embryo (fig. 1B). Then, most of the nuclei undergo microtubule-dependent peripheral migration, to divide synchronously another 4 times (round 10-13) at the cortex (Foe and Alberts 1983). Some of the nuclei travel to the posterior pole of the embryo to form the pole cells (fig. 1C and D), precursors for the adult germ cells (Raff and Glover 1989). Finally, around 6ooo syncytial blastoderm nuclei, nicely arrayed under the plasma membrane, get separated by invaginating plasma membrane furrows to form the cellular blastoderm with a polarised epithelium (Zolakar and Erk 1976; CamposOrtega and Hartenstein 2014; Mazumdar and Mazumdar 2002). The process of enclosure of each syncytial nucleus into a single cell is called cellularization (fig. 1D) (Bate and Martinez Arias 1993). Cellularization starts with interphase of cycle 14 and lasts about 65-70 min (Bate and Martinez Arias 1993).

A
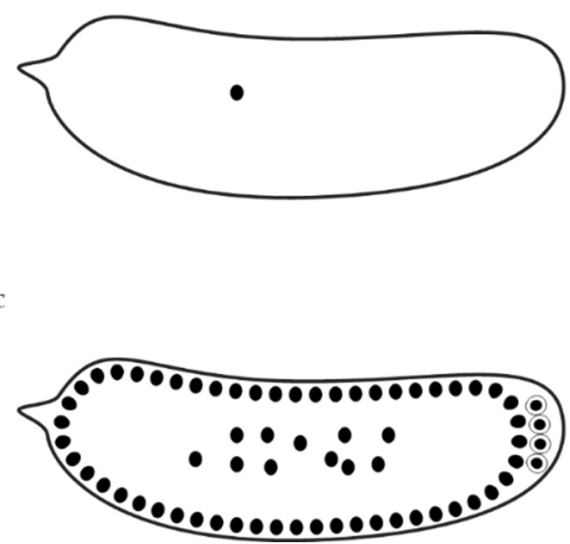

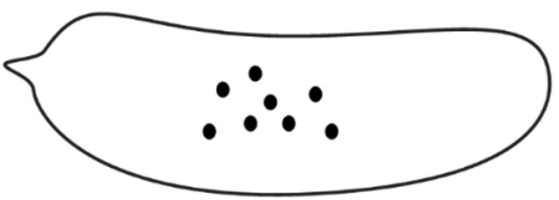

D

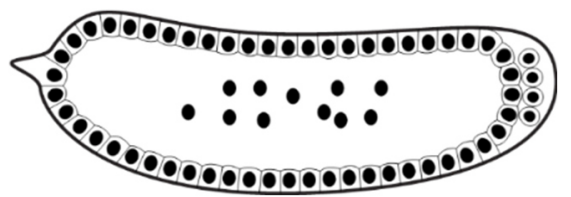

Figure 1 Simplified depiction of early Drosophila development. (A) The egg after fertilization. (B) The first rounds or mitotic division (skipping cytokinesis) take place in the interior of the embryo. (C) After the first 9 cycles the bulk of the nuclei undergo cortical migration. $(D)$ Cellularization: cell membrane invaginates between the nuclei and with basal closure all cortical nuclei are encapsuled by plasma membrane. A cellular blastoderm has formed. Picture modified from (Acharya 2014). 


\subsubsection{Metaphase furrows and actin caps during syncytial cycles}

After the first 8 preblastodermal mitotic cycles the bulk of the nuclei undergo cortical migration. At the embryonic cortex somatic buds are formed above the nuclei and their associated microtubule organizing centres, the centrosomes (Blake-Hedges and Megraw 2019). These somatic buds and the space between them are the first mark of the cortical differentiation into caps and intercap regions. During the following 4 final divisions of the syncytial blastoderm, the plasma membrane undergoes dynamic changes that are driven by the centrosome-dependent actin-microtubule network (Rikhy, Mavrakis, and Lippincott-Schwartz 2015; Blake-Hedges and Megraw 2019). During interphase microvilli- and actin-rich caps form above the nuclei. These actin caps expand and fuse as the cycle progresses to become associated with transient invaginations of the plasma membrane of approximately $8 \mu \mathrm{m}$ depths between the nuclei and cortical spindles during mitosis. These pseudocleavage or metaphase furrows regress in telophase and actin becomes reorganized in the caps leaving out the intercap region (Sullivan, Fogarty, and Theurkauf 1993; Stevenson et al. 2002). The formation of actin caps is dependent on the SCAR-activated actin-bundling Arp2/3 complex (Zallen et al. 2002).
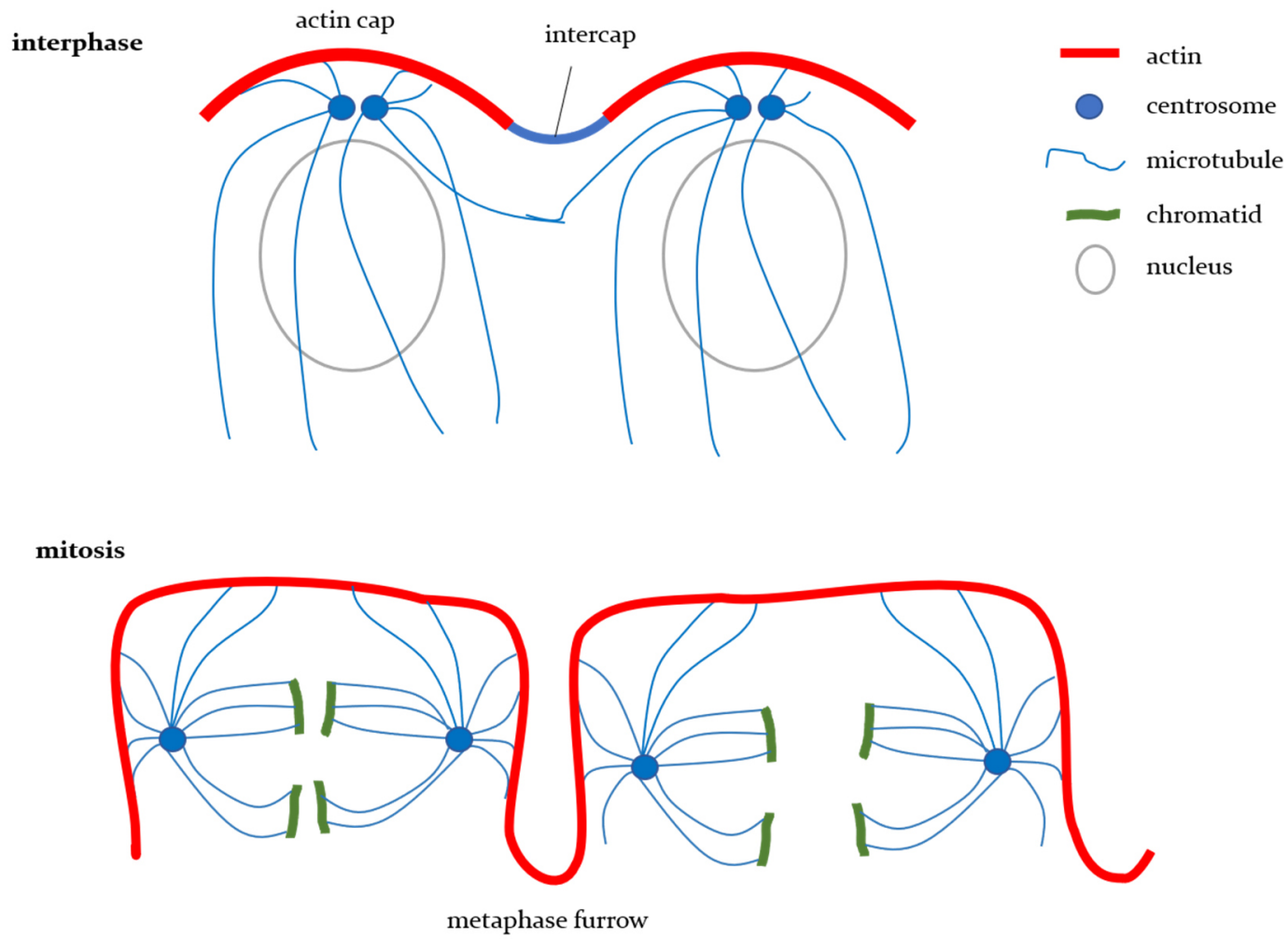

Figure 2 Cytoskeletal rearrangement during syncytial cycles. Once the nuclei reached the cortex after peripheral migration actin caps form above their associated centrosomes, which are separated by intercap regions. These actin caps rearrange for mitosis and form so called actin-rich metaphase or pseudocleavage furrows. After successful mitosis these furrows regress to restructure to actin caps and intercap region. Picture modified from (Acharya 2014). 


\subsubsection{Phases of the cellularization process}

Cellularization is a specialized form of cytokinesis and may be divided into a slow and a fast phase (Merrill, Sweeton, and Wieschaus 1988; Fullilove and Jacobson 1971; Mahowald 1963). Previously the slow phase was described to be behind the first $10 \mu \mathrm{m}$ of the furrow invagination taking 35-40 min. During the fast phase the speed of membrane invaginations increases to $20 \mu \mathrm{m}$ in 15-20 min (Thomas Lecuit, Samanta, and Wieschaus 2002). More recently, however, Figard et al. found that the switch from slow to fast phase seems to occur earlier, at $5 \mu \mathrm{m}$ furrow ingression (Figard et al. 2013).

More precisely, Lecuit and Wieschaus described 4 distinct phases of cellularization (fig. 3) (T. Lecuit and Wieschaus 2000). During the first phase the leading edge of the cellularization front forms: the furrow canal (FC, $10 \mathrm{~min})$ (Fullilove and Jacobson 1971). Also, nuclei continue to elongate basally. In phase 2 ( $20 \mathrm{~min}$ ) the nuclei complete their elongation, but the cellularization front moves very slow, so that it is hardly detectable ( $5 \mu \mathrm{m}$ below the embryo surface). Phase 3 ( $20 \mathrm{~min}$ ) is characterized by a slow invagination of the plasma membrane till the basal part of the nuclei. In phase 4 (20 min) the invagination rate suddenly increases to 2 -fold and with basal closure produces $35 \mu \mathrm{m}$ long epithelial cells. During basal closure microfilament rings detach from each other after passing the basal end of the peripheral nuclei and constrict to form a continuous membrane around them (Thomas and Wieschaus 2004). 

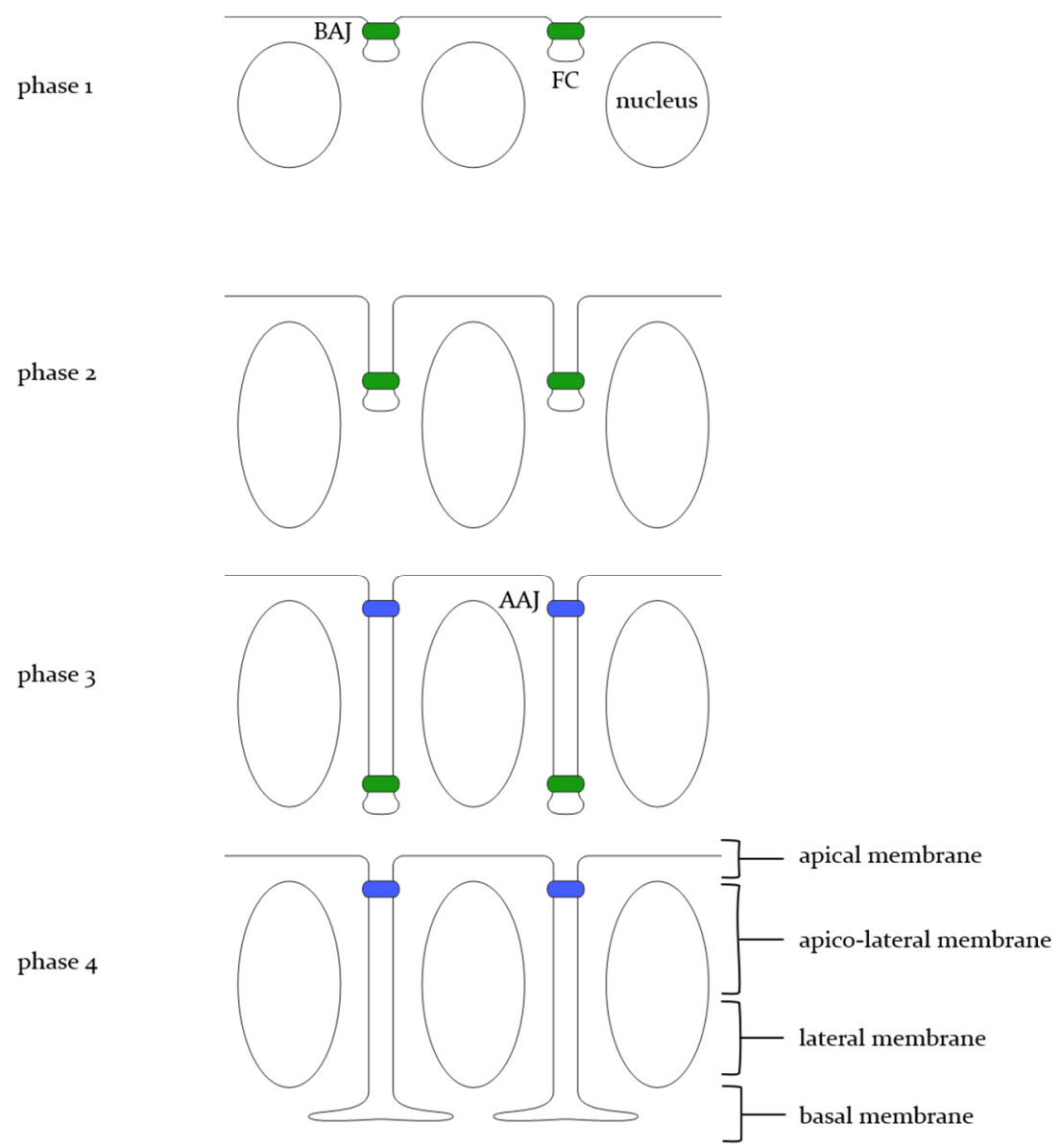

Figure 3 Phases during cellularization (Thomas Lecuit, Samanta, and Wieschaus 2002). During phase 1 the cellularization front forms: the furrow canal (FC). The nuclei elongate basally. During phase 2 all nuclei complete their elongation. The plasma membrane invaginates slowly. While phase 3 the plasma membrane ingresses slowly till it reaches the basal end of the nuclei. In phase 4 the speed of invagination increases drastically, and basal closure completes cellularization. Basal Adherens junctions (BAJ) Apical Adherens Junctions (AAJ). Picture modified from (Mazumdar and Mazumdar 2002).

\subsubsection{Components of the polarized membrane}

As mentioned earlier cytoplasmic or somatic buds form above the nuclei once they have reached the cortex. These buds are separated by intercap regions, a slight depression of the plasma membrane. During mitotic cycle 10-13 these intercap regions fold out to form metaphase furrows, separating adjacent mitotic spindles and regress in telophase to go back to the shallow invagination they evolved from. This process requires a series of dynamic cytoskeletal changes and proteins, that help regulating and stabilizing interstages. Inevitable for furrow invagination and the formation of the furrow canal are adherens junctions. Once the intercap regions ingress to define the first FC basal adherens junctions help separating the FC from the plasma membrane (Hunter and Wieschaus 2000; Hunter et al. 2002; Müller and Wieschaus 1996). The intercap region is marked by a specific set of proteins like as E-cadherin/ $\beta$-catenin complexes, Myosin II, Patj and Slam, as well as DLG/SAP97 and Scribbles. As the membrane ingression 
proceeds three distinct domains emerge. Myosin II, Slam and Patj assemble at the actin-rich FC. E-cadherin/ $\beta$-catenin complexes gather at the basal adherens junctions (BAJ) and Dlg and Scribbles at the lateral membrane, apically to the BAJ. The establishment of the BAJ is dependent on Slam (Thomas Lecuit, Samanta, and Wieschaus 2002). Nullo, however, is needed for the stabilization of the adherens junctions. Nullo gathers more outspread than Slam: to the FC, the BAJ and apically to the BAJ (Hunter and Wieschaus 2000; Thomas Lecuit 2004). During the ongoing ingression the cellularization front is marked by the three abovementioned proteins Slam, Patj and Myosin II. A number of other proteins, however, are also located to the FC. For example bottleneck, a regulator of actomyosin network, which times basal closure, is found colocalizing with Slam and others at the FC (Schejter and Wieschaus 1993; Theurkauf 1994). Or the actin-binding protein Anillin, which is required for cytokinesis and proposedly gets involved with the organization of contractile domains of the actin cytoskeleton (Sechi et al. 2017; C. M. Field and Alberts 1995; Christine M. Field et al. 2005). The actin nucleator and formin Dia is another protein, which associates with the cleavage front (Yan et al. 2013). There are many more. For a number of the proteins, however, the way they work is little understood. We know for example, where and when Slam is located, its dynamics and some interactors. We know what happens, when it is gone, but the actual task and mechanism of its function is unclear. 
A

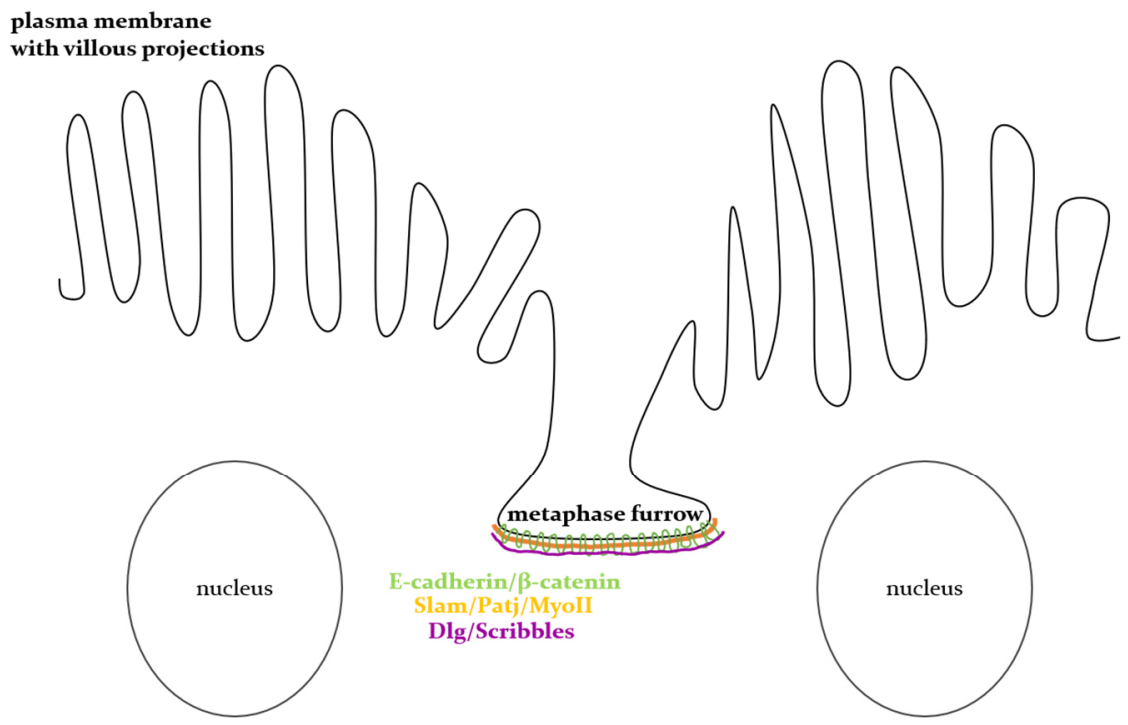

B

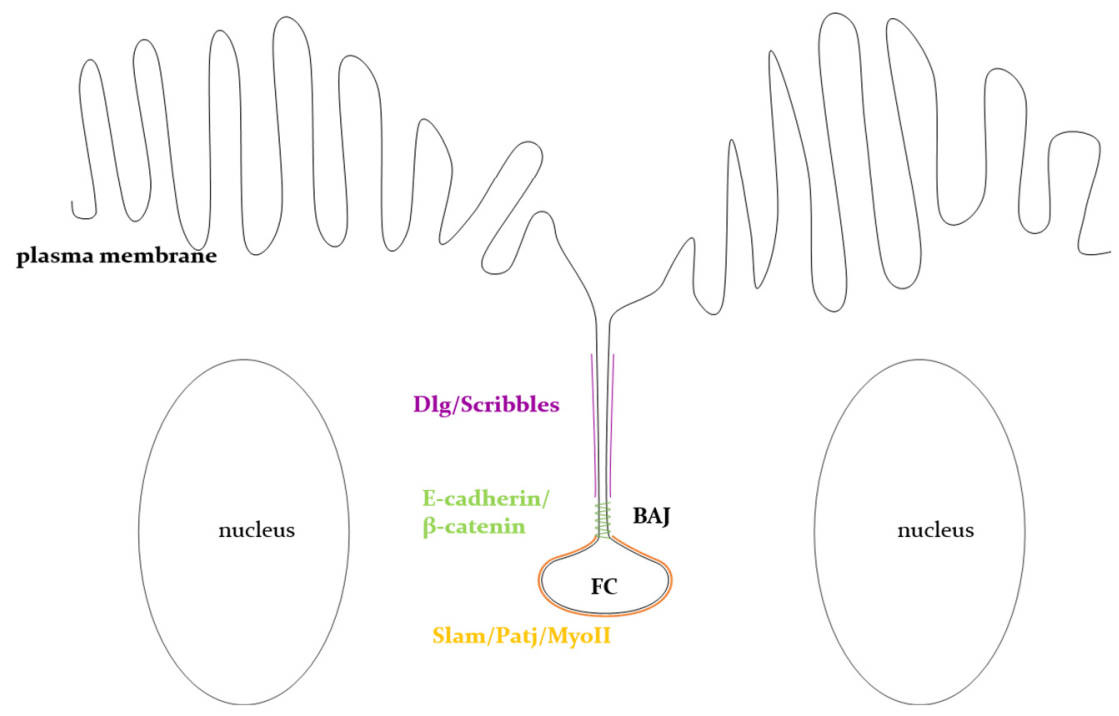

Figure 4 Components of the metaphase furrow and the early cellularization furrow. Before cellularization the plasma membrane is rich in villous projections, which gradually vanish as the membrane invaginates. (A) The metaphase furrow is marked by a set of proteins. Among them are Dlg, Scribbles, Patj, Slam, Myosin II and E-cadherin. (B) As the membrane ingresses distinct domains appear and the beforementioned proteins separate. Slam, Patj and Myoll mark the furrow canal (FC). E-cadherin is located at the basal adherens junctions (BAJ) and Dlg and Scribbles are found at the lateral membrane. Picture modified from (Thomas Lecuit 2004).

\subsubsection{Slam recruits Myosin II to the furrow canal}

During cellularization membrane invaginates to form a honeycomb-like hexagonal network, with each hexagon enclosing one of the $~ 6000$ syncytial nuclei (Mazumdar and Mazumdar 2002). Once this array has passed the basal end of the nuclei, an actomyosin driven force ensures basal closure and thus creates the first polarized epithelium of Drosophila development. 
Simultaneously with the start membrane invagination at the beginning of cellularization F-Actin and myosin accumulate at the FC (D. P. Kiehart 1990; Foe, Field, and Odell 2000; C. M. Field and Alberts 1995). The recruitment of Myosin II during cellularization was previously divided into 2 phases (B. He, Martin, and Wieschaus 2016): During the first (flow) phase a tension-driven cortical flow brings myosin to the cleavage furrows. In a second phase additional myosin at the furrow canal is recruited from the cytoplasm. The first phase requires the zygotic gene dunk, the second slam. When the plasma membrane ingression is complete and the actomyosin network reorganised to form individual rings, it constricts to close the membrane basally and form new cells. Basal closure is very similar to conventional cytokinesis and even though there are striking differences between the latter and blastoderm cellularization several proteins are alike (Schejter and Wieschaus 1993). Among them the scaffolding protein Anillin (C. M. Field and Alberts 1995), Septins (Christine M. Field et al. 2005), Rho1 (Crawford et al. 1998) and the formin Diaphanous (Dia) (Afshar, Stuart, and Wasserman 200o), which are also located to the furrow canals.

\subsection{Slow-as-molasses}

\subsection{1 slam during cellularization}

The gene slow-as-molasses (slam) is among the genes, which are transcribed early in the Drosophila embryo. Initially the RNA-polymerase II is inactive and is activated only about one hour after fertilization. This includes slam. During cellularization slam is needed for the formation and invagination of the plasma membrane and for its separation into distinct cortical domains (Thomas Lecuit, Samanta, and Wieschaus 2002; Acharya et al. 2014). It is required for Rho signalling in cellularization and, later, involved in germ cell migration (Wenzl et al. 2010; Stein et al. 2002).

This study addresses Slams biochemistry during cellularization which will be introduced in the following chapter.

There are both, maternal and zygotic gene products, that contribute to the creation of a functional cellular blastoderm during cellularization. According to current knowledge most of the cytoskeletal constituents are maternal gene products (Mazumdar and Mazumdar 2002). There are four exceptions: the genes nullo (Postner and Wieschaus 1994; Rose and Wieschaus 1992; Hunter et al. 2002; Hunter and Wieschaus 2000; Simpson and Wieschaus 1990), serendipity (Saad Ibnsouda et al. 1995; S. Ibnsouda et al. 1993; Schweisguth, Lepesant, and Vincent 1990), bottleneck (Theurkauf 1994; Schejter and Wieschaus 1993) and slam (Thomas Lecuit, Samanta, and Wieschaus 2002; Beronja and Tepass 2002). In slams case, there is not only zygotically 
derived RNA and protein. Maternally provided RNA and protein clearly contribute to the process of cellularization (Acharya et al. 2014). With only maternal slam the embryo can form furrows which ingress, but slower than in wild type. Maternally derived slam RNA and protein, however, are not able to form fully functional furrows (Yan and Großhans 2018). With maternal and zygotic slam removed, the fly embryo fails embryogenesis at a very early stage, namely to form a cellular blastoderm (Stein et al. 2002; Thomas Lecuit, Samanta, and Wieschaus 2002). Precisely, the embryo aborts forming furrow canals and basal junctions (Mazumdar and Mazumdar 2002).

Slam underlies a very strong spatiotemporal regulation. It is only detected right before and during cellularization and its rapidly degraded afterwards. Zygotic slam can be detected in low levels from mitotic cycle 11 (Yan and Großhans 2018). Before that maternal slam contributes. Slam localizes to metaphase furrows and intercap regions during the syncytial cycles. First it is detected in very low amounts, which gradually increase to reach its peak of expression in interphase of cycle 14. During cellularization Slam is localized to the furrow canal (FC), where it is tightly attached. It was found, that the restriction of Slam to the FC and its mobility during cellularization appears to be controlled by the recycling endosome and the centrosomes (Acharya et al. 2014). In the same study it was proposed that slam together with another gene, nullo, specifies the furrow.

\subsubsection{Slam and its mRNA}

Slam and its mRNA transcript have an unusual relationship. In most of the cases a protein is translated from its earlier transcribed mRNA and then leaves its place of birth to fulfil its task. Normally the mRNA that serves as the code for the emerging protein does not influence its function. There are also non-coding RNAs that are not translated into a protein and still have distinct functions in the cell. Well known are tRNAs, siRNAs or microRNAs (Bartel 2018; Laganà et al. 2015; Sharp et al. 1985). That an mRNA serves as a functional transcript and influences the function of the protein it encodes and this protein in turn guides its own mRNA, is peculiar.

Slam and its mRNA colocalize during early Drosophila development. They are found at metaphase furrows and intercap regions during syncytial cycles and to basal particles under the nuclei and are strongly enriched at the FC during cellularization (Schmidt, Lv, and Großhans 2018; Yan et al. 2017). The colocalization of Slam and its mRNA was also observed ectopically in nuf females, where the recycling endosome is unfunctional and Slam localizes apically (Yan et al. 2017; Acharya et al. 2014; Rothwell et al. 1998). Slam has an estimated half-life of 40 min, which matches its function during and the timeframe of cellularization (Acharya et al. 2014). 
Slam is mainly synthesised during only a few minutes at the switch from mitosis 13 to interphase 14 to be stable afterwards over the course of cellularization (Yan et al. 2017). By imaging the first round of translation (via TRICK assay) it has been found that Slam untranslated mRNA and Slam protein colocalize to basal particles at the onset of cellularization (James M. Halstead et al. 2015; J. M. Halstead et al. 2016). Within a few minutes after the start of cellularization all the mRNAs had their first round of translation. This indicated that parts of slam mRNA are transcribed at the furrow canal and that after the start of cellularization no new mRNA reaches the furrow (Yan et al. 2017). This is consistent with strong up-regulation of slam RNA and protein at the emerging furrow canal with steady protein levels during cellularization and gradually decreasing RNA levels during the second half of cellularization. Since the RNA is present at the FC the first half of the cellularization process but only translated the first minutes, it must be subject of some translational control (Yan et al. 2017). Intriguingly an mRNA that has an early stop codon (GFPstop-slam) does not localize to the defined basal region/FC in slam deficient embryos (Yan et al. 2017). Slam is not essential for the specification of the basal region/FC. In Slam deficient embryos there will still be a very small depression of the membrane that defines the site of ingression (Thomas Lecuit, Samanta, and Wieschaus 2002). Slam mRNA needs the protein to localize to the furrow, in contrast Slam protein has its own affinity to the furrow canal and does not need the RNA to localize. This was shown by an mRNA which has an alternative codon usage, incapable of localisation (slam[ACU]). With only slam[ACU] mRNA Slam was still able to localize to the FC. Since the slam[ACU] did not colocalize with Slam at the site of action, Slam protein levels were not sufficient to complement the slam phenotype. This was characterized by short furrows and incomplete cellularization (Yan et al. 2017). Thus, Slam protein needs its mRNA at the FC to fulfil its, yet vastly unknown, task. This interdependency of a protein, that attracts its mRNA to the site of action because it needs it for local translation to achieve sufficient amounts for its purpose is special. Additionally it has been shown by co-immunoprecipitation experiments, that Slam and its mRNA are part of a, yet uncharacterized, complex (Yan et al. 2017).

\subsubsection{Biochemistry of Slam}

The gene region of Slam (CG9506) has 2 proteins assigned (TrEMBL): Slam and LD36405p. There was evidence for an mRNA coding for 1196 amino acid Slam, which was called LD36405p (Rubin 2000). LD36405p has N-terminally 23 additional amino acids compared to Slam. In this study the 1196 or LDo6405p variant was used.

Slam is not conserved beyond flies. It is a 1196 amino acid protein, that has a calculated weight of $133 \mathrm{kDa}$. During SDS-PAGEs it runs notably at a higher weight of approximately $180 \mathrm{kDa}$ 
(Wenzl et al. 2010). In the embryo Slam is membrane associated (Thomas Lecuit, Samanta, and Wieschaus 2002). Over the span of its sequence no known domains can be assigned. It appears however, that Slam displays a class II PDZ binding motif at its C-terminus (-AVEV)(Wenzl et al. 2010). This is where the PDZ domain containing proteins RhoGEF2 and Patj putatively bind. For the recruitment of RhoGEF2, however, it was shown, that the C-terminal PDZ binding motif of Slam is not necessary, as a mutated motif does not impair RhoGEF2 localization in the embryo (Wenzl et al. 2010).

According to secondary structure prediction Slam is largely disordered. Especially the middle part calculates to be completely disordered. Only the C-terminus predicts profound structure.

\subsection{Aims and Objectives}

Despite the insights in functions and mechanism on a functional and cellular level, the exact function and biochemistry of Slam during the cellularization of the syncytial blastoderm, is little understood. In my research I focused on three aspects of Slam biochemistry:

\section{Heterologous Slam protein expression and analysis}

Apart from its PDZ domain binding motif at the far C-terminus, Slam does not display any known domains or motifs throughout its entire amino acid sequence. Structural analysis has not yet been attempted. One of the reasons is that the purification of heterologous Slam is intricate. In this study a purification strategy for heterologous Slam truncations is elaborated.

\section{Slam Interactome}

It was aimed to elaborate the role of Slam and the nature of the ribonucleoprotein complex it appears to be part of by determining new possible interactors during cellularization. This was done by the co-immunoprecipitation of Slam and putative interactors and subsequent mass spectrometric analysis.

\section{Slam post-translational modifications and stability}

This study further attempts to explore the biochemistry of Slam and its mRNA during early development of Drosophila melanogaster. Are there post-translational modifications that influence Slam? Is there more interdependency between Slam and its RNA, that we do not know of? 


\subsection{Materials}

\subsubsection{Reagents}

Unless otherwise mentioned standard reagents and chemicals were acquired from AppliChem GmbH (Darmstadt), Carl Roth GmbH (Karlsruhe), Invitrogen (Carlsbad, USA), Merck (Darmstadt), Sigma-Aldrich (St. Louis, USA) and Thermo Fisher Scientific (Waltham, USA).

\subsubsection{Media and Buffers}

\section{Buffers}

All buffers were filtered prior to use.

Recombinant Protein Purification

6x Laemmli sample buffer

$\begin{array}{ll}375 \mathrm{mM} & \text { Tris/HCl pH } 6.8 \\ 10 \% & \text { SDS } \\ 50 \% & \text { glycerol } \\ \text { o.6 M } & \text { DTT } \\ \text { 0.03\% } & \text { bromophenol blue }\end{array}$

Affinity chromatography

- Glutathione-S-Transferase (GST)

load 5omM Tris, 250mM NaCl, 5\% Glycerol pH 7.5

elution $50 \mathrm{mM}$ Tris- $\mathrm{HCl}, 10 \mathrm{mM}$ reduced glutathione, $\mathrm{pH}$ 8.0

- Histidine (His)

load $20 \mathrm{mM}$ sodium phosphate, $500 \mathrm{mM}$ sodium chloride, $30 \mathrm{mM}$ imidazole $\mathrm{pH} 7 \cdot 5$

elution $20 \mathrm{mM}$ sodium phosphate, $500 \mathrm{mM}$ sodium chloride, $500 \mathrm{mM}$ imidazole $\mathrm{pH} 7 \cdot 5$ 
AnIon-exchange chromatography (IEX)

load $\quad 50 \mathrm{mM}$ Tris, $50 \mathrm{mM}$ sodium chloride $\mathrm{pH} 7.5$

elution $\quad 50 \mathrm{mM}$ Tris, $1 \mathrm{M}$ sodium chloride $\mathrm{pH} 7.5$

\section{Gel filtration}

load $\quad 10 \mathrm{mM}$ sodium phosphate, $150 \mathrm{mM}$ sodium chloride $\mathrm{pH} 7.2$

SDS-PAGE

buffer for stacking gel

$0.5 \mathrm{M}$ Tris/HCl

$0.4 \%$ SDS

pH 6.8

buffer for separating gel

$1.5 \mathrm{M}$ Tris/ $/ \mathrm{HCl}$

$0.4 \%$ SDS

$\mathrm{pH} 8.8$

10 x SDS-PAGE running buffer

$25 \mathrm{mM}$ Tris

$192 \mathrm{mM}$ glycine

$0.1 \%$ SDS

Western Blot

Transfer buffer

$25 \mathrm{mM} \quad$ Tris/ $/ \mathrm{HCl} \mathrm{pH} 7.5$

$192 \mathrm{mM}$ glycine

$10 \%$ methanol (vol/vol)

PBS

$130 \mathrm{mM} \quad \mathrm{NaCl}$

$7 \mathrm{mM} \quad \mathrm{Na}_{2} \mathrm{HPO}_{4}$

$3 \mathrm{mM} \quad \mathrm{NaH}_{2} \mathrm{PO}_{4}$

$\mathrm{pH} \quad 7.4$

PBST

$\begin{array}{ll}130 \mathrm{mM} & \mathrm{NaCl} \\ 7 \mathrm{mM} & \mathrm{Na}_{2} \mathrm{HPO}_{4} \\ 3 \mathrm{mM} & \mathrm{NaH}_{2} \mathrm{PO}_{4} \\ 0.1 \% & \text { Tween 20 } \\ \mathrm{pH} & 7.4\end{array}$


Blocking buffer

Immunoprecipitation

Immunoprecipitation (IP) Buffer

$$
\begin{array}{ll}
1 \mathrm{X} & \text { PBS } \\
5 \% & \text { milk powder }
\end{array}
$$

$\begin{array}{ll}50 \mathrm{mM} & \text { Tris/ } \mathrm{HCl} \mathrm{pH} \mathrm{7.5} \\ 75 \mathrm{mM} & \mathrm{NaCl} \\ 1 \mathrm{mM} & \mathrm{MgCl} \\ 0.05 \% & \mathrm{NP}-40 \\ 100 \mathrm{mM} & \text { Sucrose } \\ 1 \mathrm{mM} & \text { DTT } \\ 1 \mathrm{mM} & \text { AEBSF } \\ 1 \mathrm{x} & \text { cOmplete }{ }^{\mathrm{TM}} \text { mini protease inhibitor } \\ & \text { tablets (Roche) }\end{array}$

Radioimmunoprecipitation Assay (RIPA) buffer

$\begin{array}{ll}10 \mathrm{mM} & \text { Tris/HCl } \mathrm{pH} 7 \cdot 5 \\ 150 \mathrm{mM} & \mathrm{NaCl} \\ 0.1 \% & \text { SDS } \\ 1 \% & \text { Triton X-10o } \\ 1 \% & \text { Deoxycholate } \\ 5 \mathrm{mM} & \text { EDTA } \\ 1 \mathrm{mM} & \text { AEBSF } \\ 1 \mathrm{x} & \text { cOmplete }{ }^{\mathrm{TM}} \text { mini protease inhibitor } \\ & \text { tablets (Roche) }\end{array}$

$\begin{array}{lll}\text { Radioimmunoprecipitation Assay (-) } & 10 \mathrm{mM} & \text { Tris/HCl pH 7.5 } \\ & 75 \mathrm{mM} & \mathrm{NaCl} \\ & 1 \% & \text { Triton X-10o } \\ 1 \% & \text { Deoxycholate } \\ 5 \mathrm{mM} & \text { EDTA } \\ 1 \mathrm{mM} & \text { AEBSF } \\ 1 \mathrm{~m} & \text { cOmplete }{ }^{\mathrm{TM}} \text { mini protease inhibitor } \\ & & \text { tablets (Roche) }\end{array}$


Miniprep of plasmid DNA

Solution I

Solution III
$50 \mathrm{mM}$ Tris $/ \mathrm{HCl}$

10 mM EDTA

pH 8.o

$1 \%$ SDS

$0.2 \mathrm{M} \mathrm{NaOH}$

$3 \mathrm{M}$ potassium acetate

adjusted to $\mathrm{pH} 5.4$ with acetic acid

\section{Media}

\section{Bacterial cultures}

Media were prepared by laboratory technicians according to standard protocols (Sambrook and Russell 2001).

$\begin{array}{lll}\text { Luria-Bertani Broth (LB Broth) } & 10 \mathrm{~g} & \text { Bactotryptone } \\ & 5 \mathrm{~g} & \text { Yeast extract } \\ 10 \mathrm{~g} & \mathrm{NaCl} \\ & \text { ad } 1000 \mathrm{ml} & \mathrm{ddH} 20 \\ & & \\ \text { LB Agar plates } & 10 \mathrm{~g} & \text { Bactotryptone } \\ & 5 \mathrm{~g} & \text { Yeast extract } \\ 10 \mathrm{~g} & \mathrm{NaCl} \\ 15 \mathrm{~g} & \mathrm{Agar} \\ & \text { ad 1000 ml } & \text { ddH20 }\end{array}$

Medium was autoclaved and allowed to cool down to $55^{\circ} \mathrm{C}$. Required antibiotics were added and the solution plated into petri-dishes. The Agar plates were stored at $4^{\circ} \mathrm{C}$ until further use. 
Terrific Broth (TB)

$\begin{array}{ll}12 \mathrm{~g} & \text { Tryptone (pancreatic digest of casein) } \\ 24 \mathrm{~g} & \text { Yeast extract } \\ 9.4 \mathrm{~g} & \mathrm{~K}_{2} \mathrm{HPO}_{4} \\ 2.2 \mathrm{~g} & \mathrm{KH}_{2} \mathrm{PO}_{4} \\ 5 \mathrm{~g} & \text { glycerol } \\ \text { ad 1000 ml } & \text { ddH2O }\end{array}$

\section{Fly media}

Fly Food

$128 \mathrm{~g}$ agar was added to $10 \mathrm{~L}$ water. This was cooked to $2 \mathrm{~h}$ till the agar completely dissolved. Subsequently 400 fresh yeast, 160 g soya-bean meal and $1.28 \mathrm{~kg}$ maize meal was added to $4 \mathrm{~L}$ of water. This was mixed thoroughly and added to the agar. The mixture was cooked for 2 h. $1.28 \mathrm{~kg}$ malt extract and $350 \mathrm{~g}$ sugar-beet molasses were added to $2 \mathrm{~L}$ of water and added to the mixture. Everything was cooked for $30 \mathrm{~min}$. In the following the food was allowed to cool down to under $60^{\circ} \mathrm{C}$. $24 \mathrm{~g}$ Nipagin was dissolved in ethanol and together with $150 \mathrm{~mL}$ propionic acid added to the food. Finally, the fly food was filled into vials and allowed to solidify. The vials were then plugged and stored at $18^{\circ} \mathrm{C}$ until further use.

\section{Apple juice agar plates}

For a $4 \mathrm{~L}$ batch $70 \mathrm{~g}$ agar was dissolve in $3 \mathrm{~L}$ of water and autoclaved. The liquid agar was subsequently kept in a $60^{\circ} \mathrm{C}$ water bath. $100 \mathrm{~g}$ sugar was dissolved in $1 \mathrm{~L}$ apple juice and warmed up in a $60^{\circ} \mathrm{C}$ water bath. $6 \mathrm{~g}$ Nipagin was dissolve in $40 \mathrm{~mL}$ of ethanol (tech). The agar, the sugared apple juice and the Nipagin-solution were thoroughly mixed and filled into petri-dishes. These were stored at $4^{\circ} \mathrm{C}$ until further use.

\subsubsection{Enzymes and Kits}

\section{Enzymes}

Restriction enzymes were purchased from Thermo Fisher Scientific (Waltham, USA) and New England Biolabs (Ipswich, USA). They were applied according to manufactures guidelines.

DNase I

Lysozyme

TEVprotease
Roche

AppliChem

kindly gifted by Prof. Görlich (Göttingen) 
RNase A, DNase, and protease free (10 mg/mL)

SUMOstar

PreScission Protease

Taq Polymerase

Pfu Polymerase
Thermo Fisher Scientific

kindly gifted by Prof. Görlich (Göttingen)

kindly gifted by Jan Faix

expressed and purified in the Großhans lab

expressed and purified in the Großhans lab

Qiagen

Macherey-Nagel

Clontech

\subsubsection{Oligonucleotides}

Table 1 Primers used in this study

\begin{tabular}{|c|c|c|}
\hline Primer & Sequence $5^{\prime} \cdots 3^{\prime}$ & Details \\
\hline \multicolumn{3}{|c|}{ GST-Slam $^{1069-1196}$-His $_{6}$ cloning } \\
\hline LC16 & $\begin{array}{l}\text { atggtgatggtgatgagatctGACCTCCACGGCCC } \\
\mathrm{T}\end{array}$ & $\begin{array}{l}\text { reverse primer for insert amplification, } \\
\text { introducing BglII site }\end{array}$ \\
\hline LC2O & $\begin{array}{l}\text { CAGGGGCCCggatccagatctCTGGACGAGC } \\
\text { TGTTTGC }\end{array}$ & $\begin{array}{l}\text { forward primer for insert } \\
\text { amplification, introducing BglII site }\end{array}$ \\
\hline JG158 & CGTATTGAAGCTATCCCAC & $\begin{array}{l}\text { sequencing primer for pGEX-6O-H } \\
\text { and pGEX-6P-1 }\end{array}$ \\
\hline \multicolumn{3}{|c|}{ GST-Slam ${ }^{24-527}$ cloning } \\
\hline $\mathrm{SG}_{5}$ & $\begin{array}{l}\text { GGGATCCCCGGAATTCATGGTCCTAAGC } \\
\text { AATTCCACG }\end{array}$ & $\begin{array}{l}\text { forward primer for } \text { insert } \\
\text { amplification, introducing EcoRI site }\end{array}$ \\
\hline SG6 & $\begin{array}{l}\text { GTCGACCCGGGAATTCTTACTCTAATTT } \\
\text { TGCGATGGCCTC }\end{array}$ & $\begin{array}{l}\text { reverse primer for insert amplification, } \\
\text { introducing EcoRI site }\end{array}$ \\
\hline \multicolumn{3}{|c|}{ GST-Slam ${ }^{1069-1196}$ cloning } \\
\hline $\mathrm{SG}_{7}$ & $\begin{array}{l}\text { GGGATCCCCGGAATTCCTGGACGAGCTG } \\
\text { TTTGCCAA }\end{array}$ & $\begin{array}{l}\text { forward primer for insert } \\
\text { amplification, introducing EcoRI site }\end{array}$ \\
\hline
\end{tabular}




\begin{tabular}{|c|c|c|}
\hline SG8 & $\begin{array}{l}\text { GTCGACCCGGGAATTCTTAGACCTCCAC } \\
\text { GGCCCTCC }\end{array}$ & $\begin{array}{l}\text { reverse primer for insert amplification, } \\
\text { introducing EcoRI site }\end{array}$ \\
\hline \multicolumn{3}{|c|}{ GST-His ${ }_{6}$ Slam truncations } \\
\hline $\mathrm{LC}_{5}$ & $\begin{array}{l}\text { CAGGGGCCCggatccagatctATGCCAGAAA } \\
\text { GCCAC }\end{array}$ & $\begin{array}{l}\text { forward primer for insert amplification } \\
\text { in Slam }{ }^{1-300} \text {-cloning, introducing BglII } \\
\text { site }\end{array}$ \\
\hline LC6 & $\begin{array}{l}\text { atggtgatggtgatgagatctGGCTGCCATGTCAC } \\
\text { C }\end{array}$ & $\begin{array}{l}\text { reverse primer for insert amplification } \\
\text { in Slam }{ }^{1-300} \text { and Slam }{ }^{24-300} \text {-cloning, } \\
\text { introducing BglII site }\end{array}$ \\
\hline $\mathrm{LC}_{7}$ & $\begin{array}{l}\text { CAGGGGCCCggatccagatctATGGTCCTAAG } \\
\text { CAATTCC }\end{array}$ & $\begin{array}{l}\text { forward primer for insert amplification } \\
\text { in Slam }{ }^{24-250} \text {-, Slam } \\
\text { Slam } \\
\text { Sl-20o } \text { Slam }^{24-450} \text {, } \\
\text { introducing BglII site }\end{array}$ \\
\hline $\mathrm{LC}_{7.2}$ & $\begin{array}{l}\text { AGGGGCCCggatccagatctATGGTCCTAAGC } \\
\text { AATTCCACG }\end{array}$ & $\begin{array}{l}\text { forward primer for insert amplification } \\
\text { in Slam }{ }^{24-350} \text { - and Slam }{ }^{24-400} \text {-cloning, } \\
\text { introducing BglII site }\end{array}$ \\
\hline LC8 & $\begin{array}{l}\text { atggtgatggtgatgagatctCATCTCATTTTGCA } \\
\text { GCTGAAC }\end{array}$ & $\begin{array}{l}\text { reverse primer for insert amplification } \\
\text { in Slam }{ }^{24-250} \text {-cloning, introducing BglII } \\
\text { site }\end{array}$ \\
\hline LC9 & $\begin{array}{l}\text { atggtgatggtgatgagatctCTCCATCTCCGGCA } \\
\text { G }\end{array}$ & $\begin{array}{l}\text { reverse primer for insert amplification } \\
\text { in Slam }{ }^{24-200} \text {-cloning, introducing BglII } \\
\text { site }\end{array}$ \\
\hline LC10 & $\begin{array}{l}\text { atggtgatggtgatgagatctCGATCCCGGCTCCA } \\
\text { G }\end{array}$ & $\begin{array}{l}\text { reverse primer for insert amplification } \\
\text { in Slam }{ }^{24-350} \text {-cloning, introducing BglII } \\
\text { site }\end{array}$ \\
\hline LC11 & $\begin{array}{l}\text { atggtgatggtgatgagatctCAGGCATCGGCGG } \\
\text { GG }\end{array}$ & $\begin{array}{l}\text { reverse primer for insert amplification } \\
\text { in Slam }{ }^{24-400} \text {-cloning, introducing BglII } \\
\text { site }\end{array}$ \\
\hline LC12 & $\begin{array}{l}\text { atggtgatggtgatgagatctAAACATTTGGCTAT } \\
\text { CCGC }\end{array}$ & $\begin{array}{l}\text { reverse primer for insert amplification } \\
\text { in Slam }{ }^{24-450} \text {-cloning, introducing BglII } \\
\text { site }\end{array}$ \\
\hline $\mathrm{LC}_{13}$ & $\begin{array}{l}\text { atggtgatggtgatgagatctCTCAAGTAGCGGTT } \\
\text { G }\end{array}$ & $\begin{array}{l}\text { reverse primer for insert amplification } \\
\text { in Slam }{ }^{24-500} \text {-cloning, introducing BglII } \\
\text { site }\end{array}$ \\
\hline
\end{tabular}




\begin{tabular}{|c|c|c|}
\hline $\mathrm{LC}_{14}$ & $\begin{array}{l}\text { atggtgatggtgatgagatctCTCTAATTTTGCGA } \\
\text { TGGC }\end{array}$ & $\begin{array}{l}\text { reverse primer for insert amplification } \\
\text { in Slam }{ }^{24-527} \text {-cloning, introducing BglII } \\
\text { site }\end{array}$ \\
\hline $\mathrm{LC}_{15}$ & $\begin{array}{l}\text { CAGGGGCCCggatccagatctTGCTCGAACG } \\
\text { ATCCGATG }\end{array}$ & $\begin{array}{l}\text { forward primer for insert amplification } \\
\text { in Slam }{ }^{750-1196} \text {-cloning, introducing } \\
\text { BglII site }\end{array}$ \\
\hline LC16 & $\begin{array}{l}\text { atggtgatggtgatgagatctGACCTCCACGGCCC } \\
\mathrm{T}\end{array}$ & $\begin{array}{l}\text { reverse primer for insert amplification } \\
\text { in Slam }{ }^{75^{-1196}-} \text {, Slam }{ }^{800-1196} \text {-, Slam }{ }^{81-1196} \text { - } \\
\text { and Slam }{ }^{850-1196} \text {-cloning, introducing } \\
\text { BglII site }\end{array}$ \\
\hline $\mathrm{LC}_{17}$ & $\begin{array}{l}\text { CAGGGGCCCggatccagatctGAGCGCCCTC } \\
\text { GTCGC }\end{array}$ & $\begin{array}{l}\text { forward primer for insert amplification } \\
\text { in Slam }{ }^{800-1196} \text {-cloning, introducing } \\
\text { BglII site }\end{array}$ \\
\hline $\mathrm{LC}_{18}$ & $\begin{array}{l}\text { CAGGGGCCCggatccagatctGTGATCCGGG } \\
\text { AGGATCAT }\end{array}$ & $\begin{array}{l}\text { forward primer for insert amplification } \\
\text { in Slam }{ }^{81-1196} \text {-cloning, introducing BglII } \\
\text { site }\end{array}$ \\
\hline $\mathrm{LC}_{19}$ & $\begin{array}{l}\text { CAGGGGCCCggatccagatctGATGAGAGTC } \\
\text { CGACCGGC }\end{array}$ & 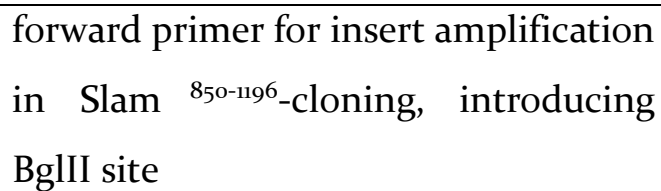 \\
\hline
\end{tabular}

\subsubsection{Plasmids}

Table 2 Plasmids used in this study

\begin{tabular}{|l|l|l|}
\hline Plasmid & Details & Source \\
\hline pGEX-6-OH & GST-His6 fusion protein & Prof. Dr. Jörg Großhans \\
\hline pGEX-6P-1 & GST-fusion protein & Amersham \\
\hline attB-slam8,5-2intron & Slam genomic region & Prof. Dr. Jörg Großhans \\
\hline OT-Slam & Full length cDNA (LD36405) & Prof. Dr. Jörg Großhans \\
\hline pGEX-slam ${ }^{23-527}$ & GST-Slam ${ }^{23-527}$ fusion protein & \\
\hline pGEX-slam ${ }^{1069-1196}$ & GST-Slam ${ }^{1069-1196}$ fusion protein & \\
\hline LD 36405 slam & Slam cDNA & \\
\hline
\end{tabular}




\subsubsection{Antibodies}

Table 3 Primary antibodies used in this study

\begin{tabular}{|l|l|l|}
\hline Antibody & Raised in & Source \\
\hline Slam (aa 1-681) & guinea pig & (Brandt et al. 2006) \\
\hline$\alpha$-Tubulin & mouse & Hybridoma bank B512 \\
\hline SHMT & rabbit & \\
\hline
\end{tabular}

IRDye ${ }^{\circledast}$ Secondary antibodies were purchased from LI-COR Biotechnology and used at a dilution of 1:20000 $(0.05 \mu \mathrm{g} / \mathrm{ml})$ as noted in table 4 .

Table 4 Secondary antibodies used in this study

\begin{tabular}{|l|l|}
\hline Antibody & Dilution \\
\hline $\begin{array}{l}\text { IRDye } \\
\text { Secondary Antibody }\end{array}$ & $1: 20000$ \\
\hline IRDye $^{\circledast} 800 C W$ Donkey anti-Guinea Pig IgG & $1: 20000$ \\
\hline IRDye $^{\circledast} 680$ RD Donkey anti-Mouse IgG & $1: 20000$ \\
\hline
\end{tabular}

\subsubsection{Bacterial Cell Lines}

$\mathrm{DH}_{5} \alpha$ was used for plasmid amplification and Arctic Express ( $\left.\mathrm{DE}_{3}\right)$ for protein purification purposes.

\begin{tabular}{|c|c|}
\hline Host strain & Genotype \\
\hline $\begin{array}{l}\text { ArcticExpress (DE3) Competent Cells } \\
\text { (Agilent Technologies) }\end{array}$ & 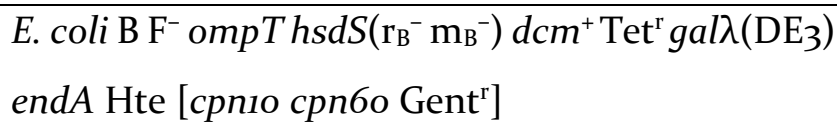 \\
\hline $\mathrm{DH}_{5} \alpha$ & $\begin{array}{l}\mathrm{F}^{-} \phi 80 \text { lacZ } \Delta \mathrm{M}_{15} \Delta(\text { lacZYA-argF }) \mathrm{U}_{169} \text { recA1 endA1 } \\
h s d \mathrm{R}_{17}\left(\mathrm{r}_{\mathrm{K}}^{-}, \mathrm{m}_{\mathrm{K}}^{+}\right) \text {phoA supE44 } \lambda^{-} \text {thi-1 gyrA96 relA1 }\end{array}$ \\
\hline
\end{tabular}

\subsubsection{Fly Stocks}

Table 5 Fly stocks used in this study

\begin{tabular}{|l|l|l|}
\hline Name & Genotype & Source/Lab Serial Nr. \\
\hline Oregon-R & $+/+$ & A401 \\
\hline GFP-Slam & w ; GFP-Slam $[68]\{w+\}$ & Ho87 \\
\hline
\end{tabular}




\subsubsection{Others}

\section{Reagents and Materials}

Agarose

BSA/Albumin Fraction V

DanKlorix

DNA ladder ( $1 \mathrm{~kb})$

dNTPs

Dynabead MyOne Streptavidin T1

Falcon tubes ( $15 \mathrm{ml}, 50 \mathrm{ml})$

Filter papers (Ø $110 \mathrm{~mm})$

Fly cages (

Fly vials

Forceps

Formaldehyde (37\%)

Glass homogenizer

Glass pipettes $(5 \mathrm{ml}-25 \mathrm{ml})$

Glass slides

Green Taq buffer (10x)

Milk powder

Parafilm M

Pasteur pipettes

Petri dishes

Phenol/Chloroform/Isopropanol

Pipetman $(2 \mu \mathrm{l}, 10 \mu \mathrm{l}, 200 \mu \mathrm{l}, 1000 \mu \mathrm{l})$

Pipette tips

Pre-stained Protein Ladder

Protease Inhibitor Cocktail - Complete Mini (EDTA free)

Protran Nitrocellulose Membrane

Ribolock RNase inhibitor $(40 \mathrm{O} / \mu \mathrm{L})$

Sodium Hypochlorite solution (15\%)

TritonX-10o

Tween 20

Viva spin
Invitrogen

Roth

Thermo Scientific

Thermo Scientific

Invitrogen

BD Falcon

Macherey-Nagel

Greiner

Sigma-Aldrich

B. Braun Biotech International

Silber Brandt

Thermo Scientific

Thermo Scientific

Sucofin

Bemis

Brandt

Greiner

Roth

Gilson

Sarstedt

Thermo Scientific

Roche

Amersham

Thermo Fisher Scientific

Sigma-Aldrich

Roth

Roth

Sartorius 
Whatman $3 \mathrm{~mm}$ blotting paper

Yeast

\section{Equipment}

Äkta pure

Centrifuges

chromatography columns

Concentrator

DynaMag - 5 Magnet

DynaMag - Spin Magnet

Electrophoresis Constant Power Supply

NanoDrop 2000c

Odyssey CLx Infrared imaging system

Thermomixer

Trans-Blot Semidry

Ultrasonics Sonifier-45o

\subsubsection{Software}

Adobe Illustrator CS6

Adobe Photoshop CS6

Image Studio

Microsoft Excel 2008

Microsoft Power Point 2008

Microsoft Word 2008

NanoDrop 2000

Scaffold Viewer v4.8.4

SeqBuilder

Zotero
GE healthcare

Dr. Oetker
GE Healthcare Life Sciences

Eppendorf

GE Healthcare

Eppendorf

Invitrogen

Invitrogen

Pharmacia

Thermo Scientific

LI-COR Biosciences

Eppendorf

Biorad

Branson

Adobe

Adobe

LI-COR Biosciences

Microsoft

Microsoft

Microsoft

Thermo Fischer Scientific

Proteome Software Inc.

DNASTAR

Roy Rosenzweig Center for History and New Media 


\subsection{Methods}

\subsubsection{Molecular Biology}

\subsubsection{Molecular Cloning}

Unless otherwise stated, molecular cloning techniques were adapted from Sambrook and Russel, 2001.

\section{Cloning of Slam truncations}

All constructs containing truncated slam were established using the OT-Slam plasmid as template. OT-Slam includes the full-length cDNA of Slam clone $\mathrm{LD}_{3} 6405$ (Rubin 2000).

\section{GST-Slam ${ }^{1069-1196}$}

To create a GST-tagged C-terminal fusion protein, the slam sequence for aa1o69-1196 was amplified from the template plasmid (OT-Slam) using the primer pair SG7 and SG8, introducing EcoRI restriction sites. pGEX-6P1- was EcoRI digested. The amplicon was introduced to the destination plasmid pGEX-6p-1 employing in-fusion cloning technique. The sequence was confirmed through Sanger sequencing using the primer JG158.

\section{GST-Slam ${ }^{24-527}$}

The slam DNA sequence for aa24-527 was amplified from template plasmid OT-Slam using the primer pair SG6 and SG7, simultaneously introducing an EcoRI restriction sites. pGEX-6P1- was EcoRI digested. The amplicon was introduced to pGEX-6P-1 though the EcoRI restriction site employing the in-fusion cloning technique. Sanger sequencing (primer JG158) results confirmed the sequence.

\section{GST-Slam ${ }^{1069-1196-H i s 6}$}

The Slam ${ }^{1069-1196}$ DNA sequence was amplified from the template plasmid (OT-Slam) using the primer pair LC20 and LC16, introducing BglII restriction sites. The destination plasmid (pGEX$6 \mathrm{OH})$ was BglII digested. The insert was cloned into pGEX-6OH using in-fusion cloning. The sequence was confirmed through Sanger sequencing employing the primer JG158. (Lenin Chandran) 
GST-His 6 Slam truncations

To create an expression plasmid carrying a Slam truncation with an N-terminal GST-tag and a C-terminal His6-tag the respective sequence was amplified using the primer pair mentioned in table 6. The primer pair added a BglII site to the amplicon. The destination plasmid pGEX-6O$\mathrm{H}$ was BglII digested. The linearized plasmid was fused with the respective amplicon employing in-fusion cloning. The correct sequence was verified via Sanger sequencing using the primer JG158.

Table 6 Overview of Slam truncations cloned into pGEX-6O-H. Molecular weight and primers, that have been used, are noted.

\begin{tabular}{|c|c|c|}
\hline Slam truncation & Size [kDa] & Primers used \\
\hline Slam $^{1-300}$ & 62 & $\mathrm{LC}_{5}, \mathrm{LC} 6$ \\
\hline Slam ${ }^{24-300}$ & 58 & $\mathrm{LC}, \mathrm{LC}_{7}$ \\
\hline Slam ${ }^{24-250}$ & 54 & $\mathrm{LC}_{7}, \mathrm{LC} 8$ \\
\hline Slam ${ }^{24-200}$ & 48 & $\mathrm{LC}_{7}, \mathrm{LC}_{9}$ \\
\hline Slam ${ }^{24-350}$ & 65 & $\mathrm{LC}_{7.2, \mathrm{LC} 10}$ \\
\hline Slam ${ }^{24-400}$ & 70 & $\mathrm{LC}_{7.2, \mathrm{LC} 11}$ \\
\hline Slam ${ }^{24-450}$ & 76 & $\mathrm{LC}_{7}, \mathrm{LC}_{12}$ \\
\hline Slam 24-500 & 82 & $\mathrm{LC}_{7}, \mathrm{LC}_{13}$ \\
\hline Slam ${ }^{24-527}$ & 84 & $\mathrm{LC}_{7}, \mathrm{LC}_{14}$ \\
\hline Slam ${ }^{750-1196}$ & 78 & $\mathrm{LC}_{15}, \mathrm{LC}_{16}$ \\
\hline Slam ${ }^{800-1196}$ & 73 & $\mathrm{LC}_{17}, \mathrm{LC}_{16}$ \\
\hline Slam ${ }^{811-1196}$ & 72 & LC18, LC16 \\
\hline Slam ${ }^{850-1196}$ & 67 & $\mathrm{LC}_{19}, \mathrm{LC} 16$ \\
\hline
\end{tabular}

\subsubsection{Polymerase Chain Rection}

Polymerase chain reactions (PCR) were performed using Taq or Phusion HF DNA polymerase. A standard PCR setup contained the following reagents:

\section{component}

DNA template

forward primer

reverse primer

dNTP mix

Green Taq or Phusion HF buffer

Enzyme (Taq or Phusion HF DNA polymerase)

\section{final concentration}

50-200 ng

$0.5 \mu \mathrm{M}$

$0.5 \mu \mathrm{M}$

$200 \mu \mathrm{M}$

$1 \mathrm{X}$

1-2 $\mathrm{U} / 50 \mu \mathrm{L}$ reaction volume 
A routine PCR was carried out under the following thermocycling conditions:

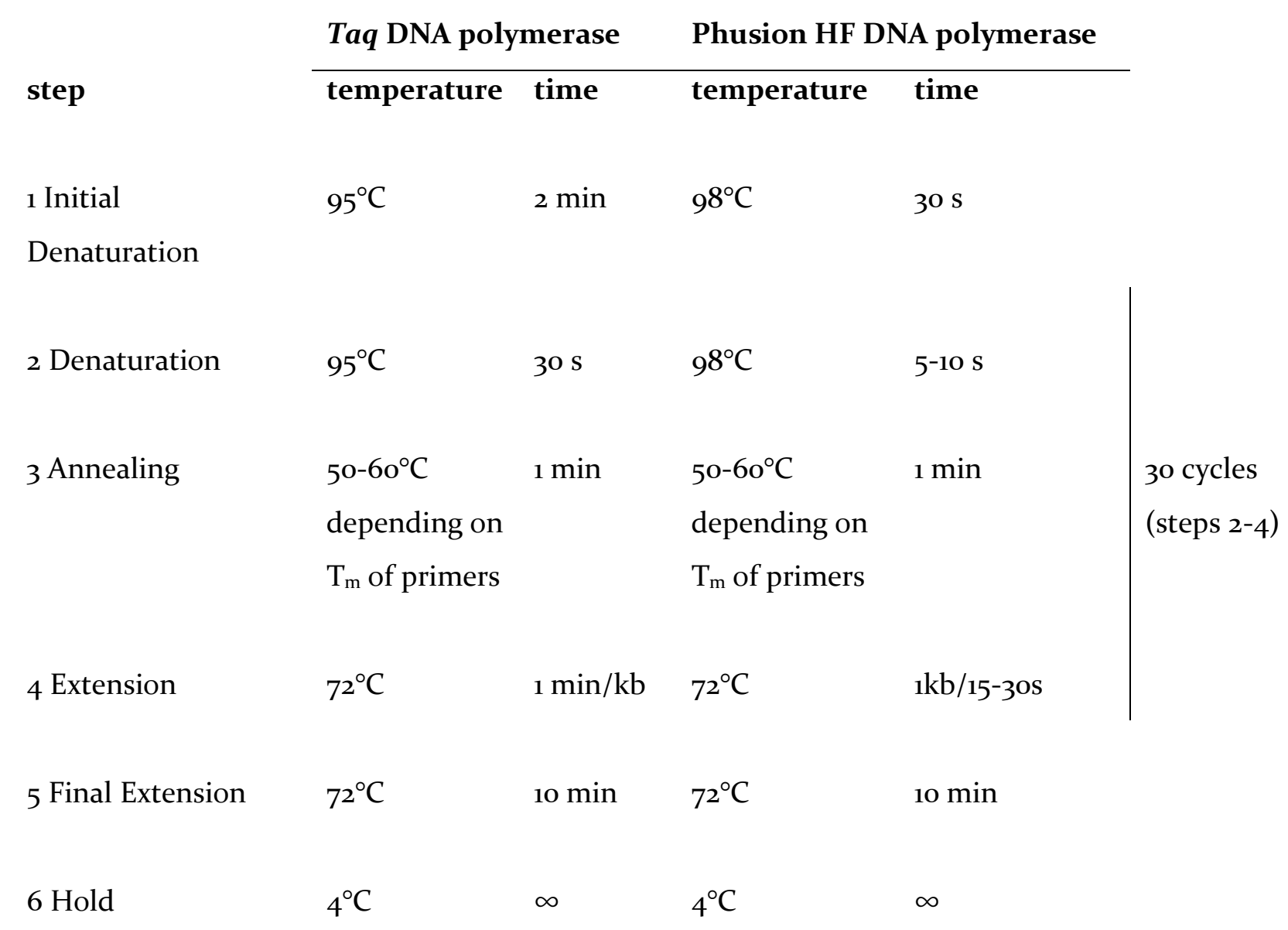

\subsubsection{In-fusion Cloning}

In-fusion cloning reactions were performed according to the manufacturer's instructions.

\subsubsection{Purification of plasmid DNA}

Amplification and purification of plasmid DNA was carried out according to Sambrook and Russel, 2001.

\subsubsection{DNA Sequencing}

Sanger sequencing was conducted by Seqlab (Göttingen). Plasmid-primer mixtures were prepared according to their instructions. 


\subsubsection{Biochemistry}

\subsubsection{Recombinant Protein Purification}

Unless otherwise stated, for recombinant protein expression E. coli ArcticExpress (DE3) cells were transformed by heat pulse with the respective protein expression plasmid. The cells were incubated overnight at $37^{\circ} \mathrm{C}$ on agar plates (containing gentamycin and ampicillin). Transformants were picked and grown overnight at $37^{\circ} \mathrm{C}$ in $2 \mathrm{YT}$ medium supplemented with gentamycin and ampicillin for selection of the expression plasmid. The optical density $\left(\mathrm{OD}_{600}\right)$ was adjusted to o.1 using the overnight cultures. The cells were grown without antibiotic selection at $30^{\circ} \mathrm{C}$ to an $\mathrm{OD}_{600}$ of 0.3. They were then further incubated at $12^{\circ} \mathrm{C}$ until they reached an $\mathrm{OD}_{6 о}$ of o.6. The expression of the plasmid was induced using o.1 $\mathrm{mM}$ Isopropyl $\beta$-D1thiogalactopyranoside (IPTG). Cells were further incubated at $12^{\circ} \mathrm{C}$ for $24 \mathrm{~h}$.

The cells were harvested by centrifugation at 50oorpm for $30 \mathrm{~min}$. The cell pellets were frozen in liquid nitrogen and stored at $-20^{\circ} \mathrm{C}$ until further use.

For lysis the pellet was resuspended in lysis buffer (50mM Tris, $150 \mathrm{mM} \mathrm{NaCl}, 5 \%$ glycerol, $\mathrm{pH} 7.5$; supplemented with cOmplete Mini protease inhibitor cocktail, Roche) and incubated 30 min on ice with $1 \mathrm{mg} / \mathrm{mL}$ lysozyme and a spatula tip of DNase (Roche). The cells were sonicated (Branson sonifier, standard disruptor horn) 4 Xios at an output level of 6 and 50\% duty cycle. Between each cycle the lysate was cooled down on ice for $1 \mathrm{~min}$. The cells were centrifuged at 15 ooorpm and $4^{\circ} \mathrm{C}$ for $20 \mathrm{~min}$. The soluble fraction (supernatant) underwent further treatment and the insoluble parts (pellet) were discarded after taking a sample for analytical purposes (SDS-PAGE).

The supernatant was filtered through a $0.45 \mu \mathrm{m}$ syringe filter and applied to the respective preequilibrated chromatography column (GE Healthcare) via an Äkta pure (GE Healthcare) pump system.

\subsubsection{Gel Filtration}

For size-exclusion chromatography (SEC) staged, cellularizing Drosophila embryos (1.5-2.5 h) were lysed in $1 \mathrm{~mL}$ RIPA buffer (10 mM Tris/ $\mathrm{HCl}$ pH 7.5, $150 \mathrm{mM} \mathrm{NaCl}, 0.1 \%$ SDS, $1 \%$ Triton X100, $1 \%$ deoxycholate, $5 \mathrm{mM}$ EDTA, $1 \mathrm{mM}$ AEBSF, $1 \times$ protease inhibitor cocktail (Roche)). After clarification of the crude lysate, $250 \mu \mathrm{L}$ of the sample was applied to a preequilibrated Superdex 200 (HR 10/30) column (GE Healthcare) using an Äkta (pure) pump system (GE Healthcare). The flow rate was adjusted to $500 \mu \mathrm{L} / \mathrm{min}$ and $1 \mathrm{~mL}$ fractions were collected. The protein of 
$400 \mu \mathrm{L}$ of each fraction was acetone precipitated and redissolved in $30 \mu \mathrm{L} 1 \mathrm{x}$ Laemmli buffer. The samples were analysed by SDS-PAGE and Western blot.

\subsubsection{Western Blot}

Unless otherwise stated the marker used for SDS-PAGES, that were planned to undergo western blot procedure, was PageRuler ${ }^{\mathrm{TM}}$ Plus Prestained Protein Ladder (10 to $250 \mathrm{kDa}$, see figure 6). After separation by SDS-PAGE, proteins were blotted onto a nitrocellulose membrane by semidry transfer. Filter papers and membrane were soaked in transfer buffer and assembled according to . Air bubbles were removed. The proteins were transferred $90 \mathrm{~min}$ at $60 \mathrm{~V} / \mathrm{gel}$.

After transfer the membrane was blocked in $5 \%$ milk powder (o.5g milk powder in 1omL freshly made PBS buffer) for at least $35 \mathrm{~min}$ at RT (room temperature). Then, the primary antibody was added (gp-slam 1:50oo). This was incubated at RT for $2 \mathrm{~h}$ or o/n at $4^{\circ} \mathrm{C}$ shaking. The membrane was rinsed 3x with PBST buffer and washed 4x 10 min each shaking. The membrane was incubated with the secondary antibody (IRDye ${ }^{\circledast}$ 8ooCW Donkey anti-Guinea Pig IgG at a dilution of 1:20000) light protected for 1.5 h at RT. The rinsing and washing steps were repeated. The bands were detected using the Odyssey CLx Infrared imaging system.

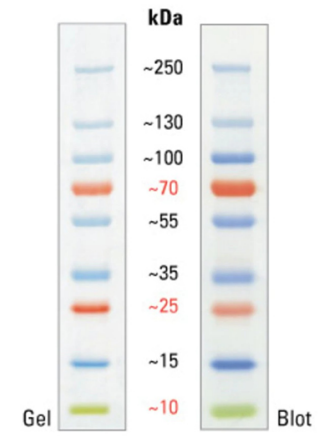

Figure 6 PageRuler ${ }^{T M}$ Plus Prestained

Protein Ladder (fishersci.de)

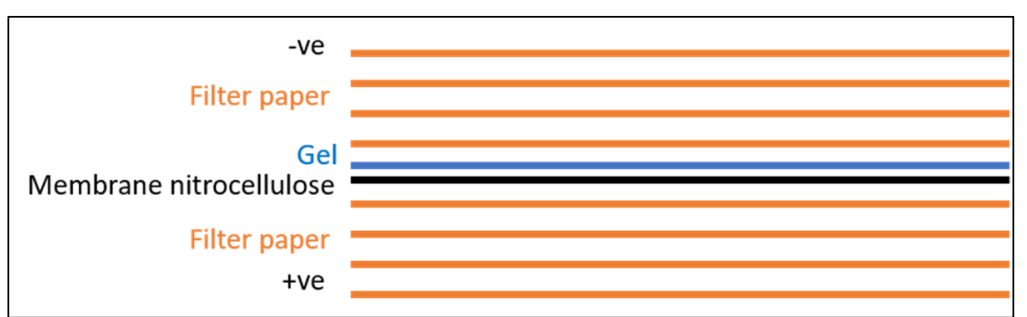

Figure 5 Assembly of Western Blot

\subsubsection{Immunoprecipitation (IP)}

For IP-experiments 300-6oo mg (for each experiment the amount GFP-Slam and WT embryos were similar) of staged fly embryos (GFP-Slam and WT) were lysed in $4 \mathrm{~mL}$ IP, RIPA(-) or RIPA buffer. Both samples, GFP-Slam and WT, were treated the same. An input sample was taken from the clarified embryonic lysate. $1.5 \mu \mathrm{L} 100 \mu \mathrm{M}$ biotinylated GFPbinder (anti-GFP nanobody, kindly gifted by Prof. Görlich) were added to the ( $20 \mu \mathrm{L}$ lysate plus $20 \mu \mathrm{L} 2 \mathrm{x}$ Laemmli) lysate. 
The GFPbinder was allowed to bind to GFP-Slam on ice for $1 \mathrm{~h}$. Meanwhile $240 \mu \mathrm{L}$ Streptavidin magnetic beads (Invitrogen) were $2 x$ PBS and 1xIP/RIPA(-) washed. The lysate was added to $120 \mu \mathrm{L}$ of the washed beads, respectively. The samples were incubated for $1 \mathrm{~h}$ on a rotary mixer at $4^{\circ} \mathrm{C}$. The beads were placed to the magnet (DynaMag, Invitrogen) for 1 min and the unbound sample was taken ( $20 \mu \mathrm{L}$ lysate plus $20 \mu \mathrm{L} 2 \mathrm{x}$ Laemmli). The residual supernatant was discarded. The tube was removed from the magnet and the beads were resuspended in $1 \mathrm{~mL}$ IP buffer. The tube was again placed to the magnet and after 1 min the supernatant was removed and discarded. The washing step was repeated twice to a total of 3 washes. After the third was the beads were again resuspended in $1 \mathrm{~mL}$ IP buffer and $1 \mu \mathrm{L}$ of SUMOstar (kindly gifted by Prof. Görlich) was added to each of the 2 tubes (GFP-Slam sample and WT sample). The tube was placed to a rotary mixer at $4^{\circ} \mathrm{C}$ and incubated to $1 \mathrm{~h}$ to allow SUMOstar to elute GFP-Slam from the beads. The tubes were placed to the magnet and after 1 min the supernatant (cleaved sample) was placed to a new tube. The protein of the cleaved sample was acetone precipitated and resuspended in $30 \mu \mathrm{L}$ of the respective buffer and $30 \mu \mathrm{L}$ Laemmli buffer. Unless otherwise mentioned the beads (bound sample) were taken up in $30 \mu \mathrm{L}$ of buffer and $30 \mu \mathrm{L}$ Laemmli. The samples were stored at $-20^{\circ} \mathrm{C}$ until further use.

\subsubsection{Protein Precipitation}

Unless otherwise mentioned, protein was precipitated using the acetone precipitation protocol. For this, the required volume of acetone was precooled to $-20^{\circ} \mathrm{C}$. Four times the sample volume of col acetone was added to the sample. The mixture was vortexed and incubated at $-20^{\circ} \mathrm{C}$ for $1 \mathrm{~h}$. Subsequently the sample was centrifuged at $14000 \mathrm{rpm}$ and $4^{\circ} \mathrm{C}$ for $10 \mathrm{~min}$. The supernatant was discarded. The protein pellet was allowed to air dry for $30 \mathrm{~min}$ and resuspended in the respective buffer.

\subsubsection{Mass Spectrometry}

Three biological replicates of IP and RIPA(-) buffer immunoprecipitation samples (3 mutant and 3 WT samples, respectively) were submitted to mass spectrometric analysis to the Scientific Core Facility Proteomics of the University Medical Center Göttingen (CF Pro). Sample proteins were separated via SDS-PAGE into 11-12 equidistant bands, which were diced, digested in-gel with trypsin, and the resulting peptides extracted. The extracts were then analysed by LC/MS/MS. Two technical replicates of each sample were measured. Proteins were identified and validated 
as described previously (Batschkus et al. 2018). Database searches were conducted against Drosophila melanogaster in TrEMBL. Scaffold Viewer v4.8.4 was employed for data evaluation (Proteome Software Inc., Portland, OR, USA). Proteins were identified using a False-Discovery Rate of $<1 \%$. The following settings were applied for identifications: protein probability threshold 99\% (prophet algorithm); peptide probability threshold 95\% (naive Bayes classifier). Proteins with less than 2 identified unique peptides were neglected. Significance in enrichment was defined as follows: enrichment in at least 2 replicates and a Benjamini-Hochberg (BH) corrected p-value of < 0.00964 (t-Test, A), <0.0003 (t-Test, B), <0.00181 (t-Test, C) and 0.03674 (ANOVA, RIPA(-) samples). Fold enrichment (FE) was calculated as the ratio of the normalized total spectral count of the identified protein in the GFPSlam and WT sample. Technical replicates were averaged.

\subsubsection{Phosphorylation Assay}

$66 \mathrm{mg}$ staged (1.5-2.5h), dechorionated wild type Drosophila embryos were lysed as using IPBuffer (including cOmplete ${ }^{\mathrm{Tw}}$ Protease Inhibitor and $1 \mathrm{mM}$ AEBSF). $10 \mu \mathrm{L}$ input sample were taken after lysis and $10 \mu \mathrm{L} 2 \mathrm{x}$ loading dye added. The mixture was incubated $5 \mathrm{~min}$ at $99^{\circ} \mathrm{C}$, spun down and stored on ice. The remaining extract was used for the assay. The Lambda Protein Phosphatase (Lambda PP) was purchased from New England Biolabs (NEB). The kit included the buffer for Protein MetalloPhosphatases (PMP) and the $\mathrm{MnCl}_{2}$ supplement.

The set up was as follows (corresponding to the manual):

\begin{tabular}{|c|c|c|}
\hline & + Lambda PP & - Lambda PP \\
\hline sample size & $100 \mu \mathrm{L}$ & $100 \mu \mathrm{L}$ \\
\hline Lambda PP & $800 \mathrm{U}$ & - \\
\hline \multirow[t]{2}{*}{ Buffer } & 1x PMP buffer & 1x PMP buffer \\
\hline & $1 \mathrm{mM} \mathrm{MnCl}{ }_{2}$ & $1 \mathrm{mM} \mathrm{MnCl}{ }_{2}$ \\
\hline
\end{tabular}

The mixtures were incubated for $30 \mathrm{~min}$ at $30^{\circ} \mathrm{C}$. Afterwards $18 \mu \mathrm{L} 6 \mathrm{x}$ loading dye were added to the sample (+LambdaPP) and the negative control (-LambdaPP). The solutions were incubated at $99^{\circ} \mathrm{C}$ for $5 \mathrm{~min}$ and spun down. The samples were loaded on an 8\% SDS-PAGE gel and run at $16 \mathrm{~mA}$ for approx. $\mathrm{hh}$. 
The proteins were blotted to a nitrocellulose membrane. The membrane was cut at $70 \mathrm{kDa}$ and stained against slam (upper part, IRDye donkey anti-guinea pig 8ooCW) and $\alpha$-tubulin (lower part, IRDye 8ooCW goat anti-mouse).

\subsubsection{Stability and RNA Assay}

Staged wt fly embryos (1.5-2.5 h) were lysed using RIPA(-) buffer. An input sample was taken, and Laemmli sample buffer added. The input sample was immediately heated to $99^{\circ} \mathrm{C}$ for $5 \mathrm{~min}$. The lysate was either treated with RNase A, RNase A (Thermo Fisher Scientific) and RNase inhibitor (Thermo Fisher Scientific) or $\mathrm{ddH}_{2} \mathrm{O}$ (control). The mixtures were incubated at RT for $10 \mathrm{~min}$. The reaction was stopped by the addition of Laemmli sample buffer. Sample proteins were separated by SDS-PAGE, blotted onto a nitrocellulose membrane and immunostained for Slam and either SHMT or tubulin as a control.

\subsubsection{Developmental Biology}

\subsubsection{Collection of Staged Fly Embryos}

Fly embryos were collected at $25^{\circ} \mathrm{C}$ for one hour on apple-juice agar plates partially coated with live yeast. The eggs were incubated at $25^{\circ} \mathrm{C}$ for another hour. This sums up to an embryo age of 1.5-2.5 h. The bulk of the yeast was removed. The embryos were dechorionated using $50 \%$ DanKlorix solution for $90 \mathrm{~s}$ ( $2,8 \mathrm{~g}$ sodium hypochlorite per $100 \mathrm{~g}$ solution). The dechorionated embryos were water-washed and dried on disposable KimTech ${ }^{\circledR}$ wipes and collected into an pre weighed Eppendorf tube. The embryos were spun down at low rotations. Subsequently the tubes were weighed, the weight of the tube was subtracted, and the weight of the embryo mass noted. The prepared embryos were frozen in liquid nitrogen and stored at $-80^{\circ} \mathrm{C} .1 \mathrm{~g}$ embryo mass are approximately 100 embryos.

\subsubsection{Lysis of Fly Embryos}

The respective amount of buffer was added to a precooled Dounce homogenizer. Frozen fly embryos were added to the homogenizer and gently lysed on ice. The crude extract was transferred to an Eppendorf tube using a cooled glass pipette and centrifuged 15 min at 15000 $\mathrm{rpm}$ and $4^{\circ} \mathrm{C}$. The emerging lipid layer was removed, and the lysate was separated from the 
debris by transferring it to a new Eppendorf tube. The lysate was then submitted to corresponding further treatment. 


\section{Results}

\subsection{Expression and purification of recombinant protein fragments of Slam}

\subsubsection{Bioinformatic analysis of Slam}

\section{Introductory remarks}

Two proteins are assigned to the gene region CG9506 (TrEMBL): Slam and LD36405p. A cDNA clone provided evidence for the mRNA of 1196 amino acid Slam or LD36405p (Rubin 200o). Both proteins have the same amino acid sequence except for a difference in the first $23 . \mathrm{LD}_{3} 6405 \mathrm{P}$ uses the first start codon and Slam the second. Slam is predicted to have 1173 amino acids and LD36405p 1196 amino acids. Unless otherwise stated Slam is assumed to consist of 1196 amino acids and the sequence information of $\mathrm{LD}_{3} 6405 \mathrm{p}$ is used, since no evidence points towards the use of the second start codon.

\section{Secondary Structure Prediction}

Secondary structure was predicted using PSIPRED (D. T. Jones 1999; Buchan and Jones 2019). To get a depiction of the results over the span of the whole protein, Slam needed to be split into two parts for structure prediction. Slam contains no known motifs or domains, which can be detected by sequence alignments. Considering the insolubility of the full-length recombinant protein, the first third of Slam shows moderate stability under purifying conditions. Hence, Slam was divided into the first $\mathrm{N}$-terminal third and the rest of the protein.

Figure 7 and 8 visualize the PSIPRED structure prediction. PSIPRED predicts mainly helices. For a few amino acids it computes beta sheets. A major part of the protein may not fold independently. The N-terminally 500 and the C-terminally 200 amino acids predict to be the most structured regions among Slam protein. The middle part may be vastly unstructured. 
20

40

50

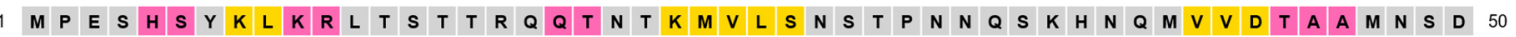

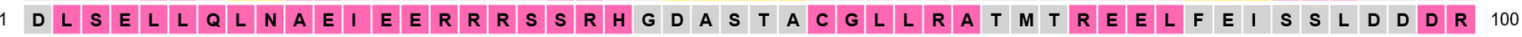

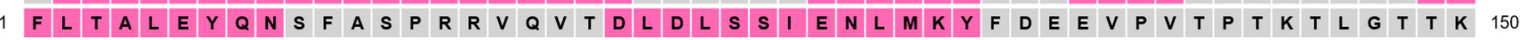

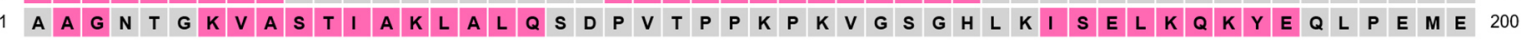

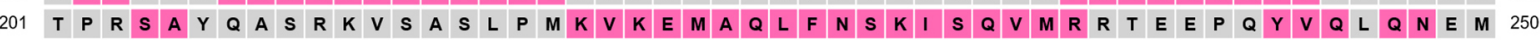

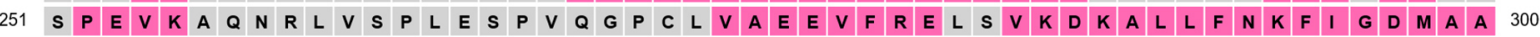

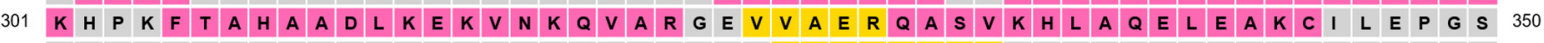

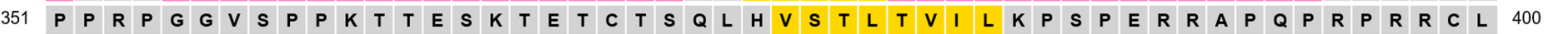

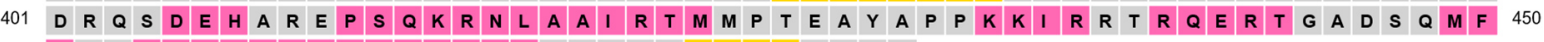

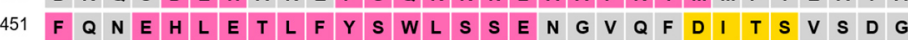

10 20 30

40

50

Strand Helix coil

Figure 7 Sequence Plot of Slam (LD36405p) displaying amino acid 1 to 480. The plot was generated using PSIPRED. Strand, helix, and coils are depicted. The N-terminal third of Slam is predicted to be rich in structure compared to the middle part (see fig. Figure 8).

20

30

40

50

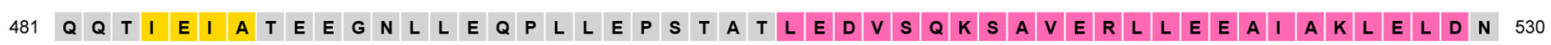

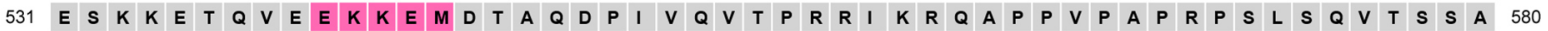

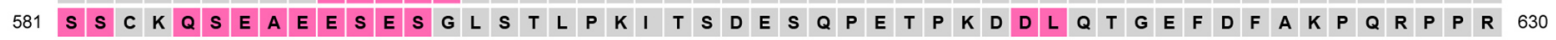

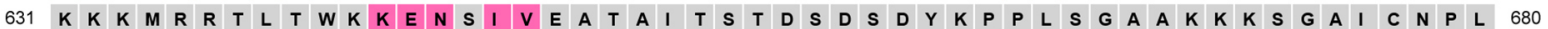

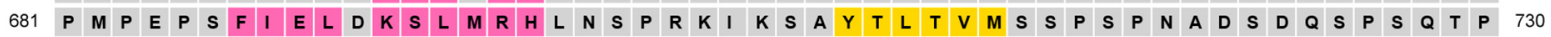

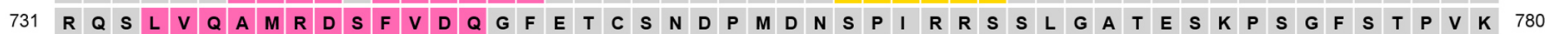

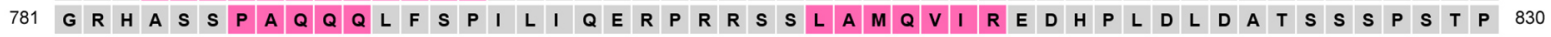

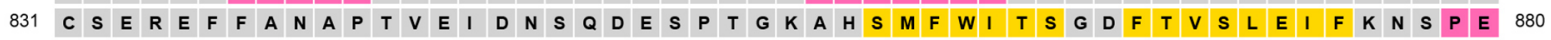

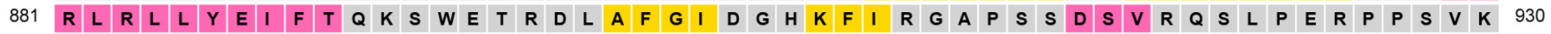

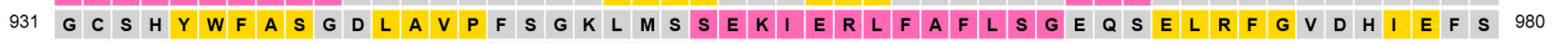

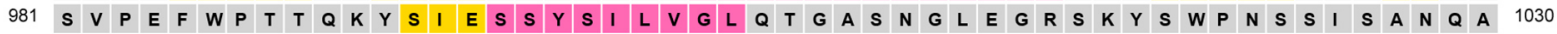

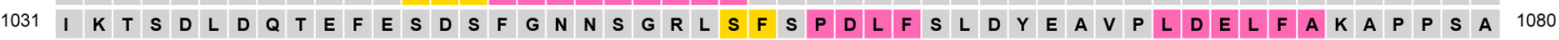

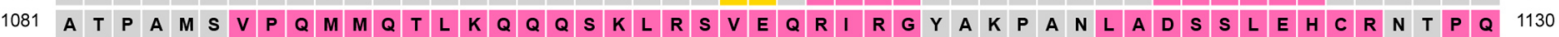

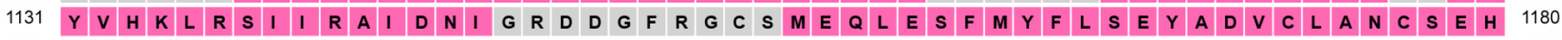
\begin{tabular}{ll|l|l|l|l|l|l|l|l|l|l|l|l|l|l|l}
1181 & M & D & K & I & L & D & T & L & M & D & R & R & A & V & E & V
\end{tabular}

$$
10
$$

30

40

50

W Helrand Coil

Figure 8 Sequence Plot of Slam (LD36405p) displaying amino acid 481 to 1196. The plot was generated using PSIPRED. Strand, helix, and coils are depicted. There is little structure predicted for the middle part of Slam. The C-terminus however predicts to predominantly be folding into a helical structure.

As indicated above, Slam is predicted to have a high degree of disorder. The plot in figure 9 shows the result of the DISOPRED3 (D. T. Jones and Cozzetto 2015) disorder prediction. Especially the middle part of the protein, between amino acid 478 and 687, and 750 and 795 , predicts to be entirely without structure. 


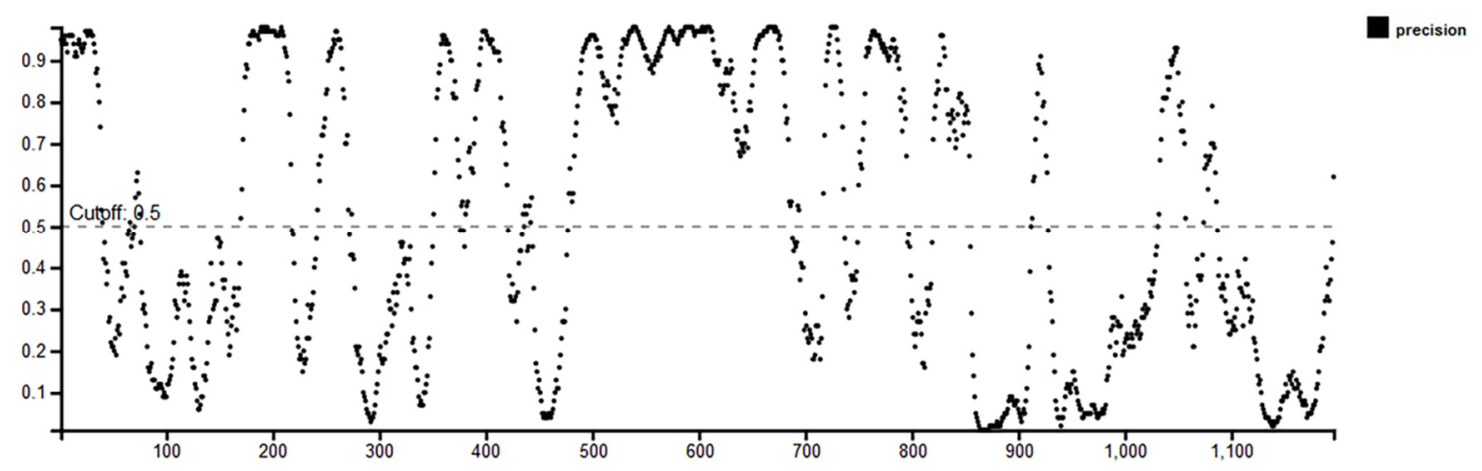

Figure 9 Slam disorder prediction using DISOPRED3. For the calculation of disorder propensity DISOPRED3 uses multiple sequence alignment employing UniRef90 database for finding homologs during a PSI-BLAST search. The plot shows the disorder confidence score against the amino acid sequence position of Slam. The grey horizontal line marks the threshold above which the respective amino acid is regarded as disordered. Slam is predicted to have a high degree of intrinsic disorder.

\section{Truncated Slam protein}

In figure 10 the consecutive numbering of Slam is depicted. The numbering starts with the first ATG of the Slam coding sequence. This means the first 23 amino acids are included and it is assumed for this thesis, that Slam consists of 1196 amino acids. Accordingly, recombinant produced protein fragments are named. Consecutive numbering of amino acids starts with the first start codon of the Slam coding sequence. 


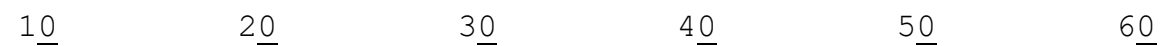
MPESHSYKLK RLTSTTRQQT NTKMVLSNST PNNQSKHNQM VVDTAAMNSD DLSELLQLNA $\begin{array}{llllll}7 \underline{0} & 8 \underline{0} & 9 \underline{0} & 10 \underline{0} & 11 \underline{0} & 12 \underline{0}\end{array}$

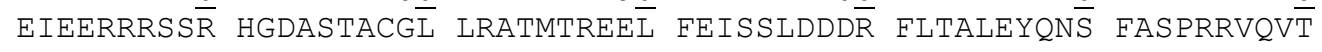

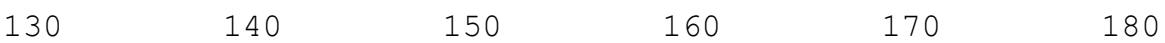
DLDLSSIENL MKYFDEEVPV TPTKTLGTTK AAGNTGKVA TIAKLALQS $\overline{\mathrm{D}}$ PVTPPKPKVG $19 \underline{0} \quad 20 \underline{0} \quad 21 \underline{0} \quad 22 \underline{0} \quad 23 \underline{0} \quad 24 \underline{0}$

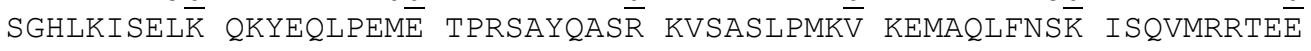
$25 \underline{0} \quad 26 \underline{0} \quad 27 \underline{0} \quad 28 \underline{0} \quad 29 \underline{0} \quad 30 \underline{0}$

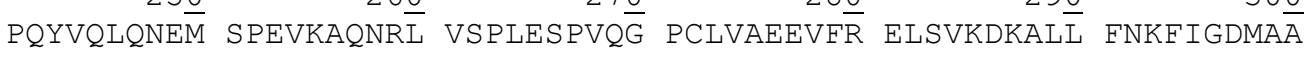

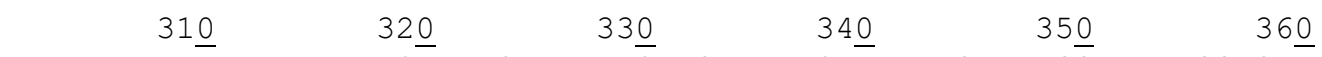

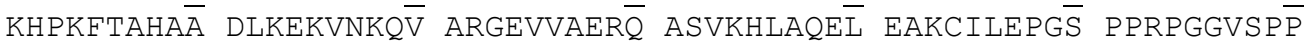
$\begin{array}{llllll}37 \underline{0} & 38 \underline{0} & 39 \underline{0} & 40 \underline{0} & 41 \underline{0} & 42 \underline{0}\end{array}$ KTTESKTETC TSQLHVSTLT VILKPSPER $\bar{R}$ APQPRPRRC $\bar{L}$ DRQSDEHARE PSQKRNLAA $\bar{I}$ $\begin{array}{llllll}43 \underline{0} & 44 \underline{0} & 45 \underline{0} & 46 \underline{0} & 47 \underline{0} & 48 \underline{0}\end{array}$

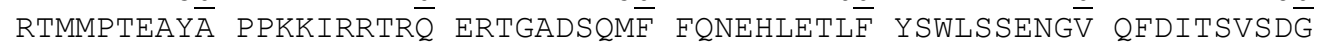
$\begin{array}{lllll}49 \underline{0} & 50 \underline{0} & 51 \underline{0} & 52 \underline{0} & 53 \underline{0}\end{array}$ QQTIEIATEE GNLLEQPLLE PSTATLEDVS QKSAVERLLE EAIAKLELDN ESKKETQVEE $55 \underline{0} \quad 56 \underline{0} \quad 57 \underline{0} \quad 58 \underline{0} \quad 59 \underline{0} \quad 60 \underline{0}$

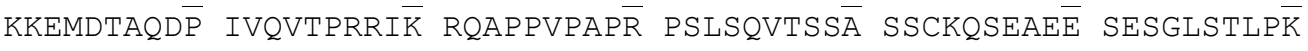
$\begin{array}{lllll}61 \underline{0} & 62 \underline{0} & 63 \underline{0} & 64 \underline{0} & 65 \underline{0}\end{array}$ ITSDESQPET PKDDLQTGEF DFAKPQRPPR KKKMRRTLTW KKENSIVEAT AITSTDSDS $\overline{\mathrm{D}}$
$\begin{array}{rrrrr}670 & 680 & 690 & 700 & 710\end{array}$

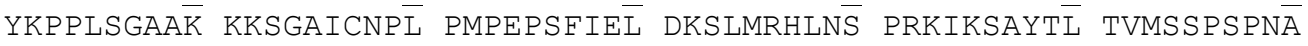

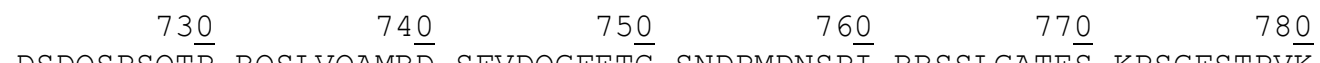
$\begin{array}{llrrr}790 & 800 & 810 & 820 & 830\end{array}$ GRHASSPAQ $\bar{Q}$ QLFSPILIQE RPRRSSLAMQ VIREDHPLDI DATSSSPST $\overline{\mathrm{P}}$ CSEREFFANA $8 \underline{0} \quad 86 \underline{0} \quad 87 \underline{0} \quad 88 \underline{0} \quad 8 \underline{0} \quad 90 \underline{0}$ PTVEIDNSQD ESPTGKAHSM FWITSGDFTV SLEIFKNSPE RLRLLYEIFT QKSWETRDL $\bar{A}$ $91 \underline{0}-92 \underline{0}-93 \underline{0} \quad 94 \underline{0} \quad 95 \underline{0} \quad 96 \underline{0}$

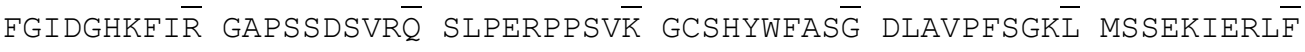

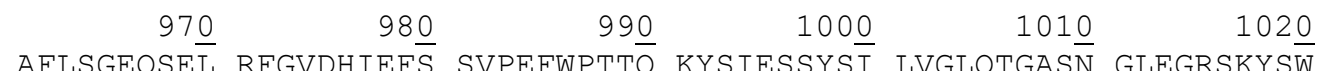
AFLSGEQSEL RFGVDHIEFS SVPEFWPTTQ KYSIESSYSI LVGLQTGASN GLEGRSKYSW
1030
1040
1050
1060
$107 \underline{0}$
$108 \underline{0}$

PNSSISANQ $\bar{A}$ IKTSDLDQTE FESDSFGNN $\bar{S}$ GRLSFSPDL $\overline{\mathrm{F}}$ SLDYEAVPL $\overline{\mathrm{D}}$ ELFAKAPPS $\overline{\mathrm{A}}$
1090
$110 \underline{0}$
$111 \underline{0}$
1120
1130
1140

ATPAMSVPQM MQTLKQQQST LRSVEQRIRG YAKPANLAD $\overline{\mathrm{S}}$ SLEHCRNTPQ YVHKLRSII $\overline{\mathrm{R}}$
$115 \underline{0}$
1160
1170
1180
1190

AIDNIGRDDG FRGCSMEQLE SFMYFLSEYĀ DVCLANCSE $\bar{H}$ MDKILDTLMD RRAVEV

Figure 10 Amino acid sequence of Slam starting with the first ATG (LD36405p) of the coding sequence (NCBI Reference Sequence: NT_033779.5). The first 23 amino acids resulting from the sequence upstream of the second ATG (Slam) are highlighted in yellow. 


\subsubsection{Purification of recombinant Slam fragments}

The full-length Slam shows little solubility (figure 11). Slam predicts to have a high degree of intrinsic disorder (figure 9), which can lead to insolubility of proteins (De Simone et al. 2012). Over the span of the protein it is the $\mathrm{N}$ - and $\mathrm{C}$-terminus, that compute to exhibit most structure. It is therefore the $\mathrm{N}$ - and C-terminus that have been chosen for first purification and crystallization attempts.

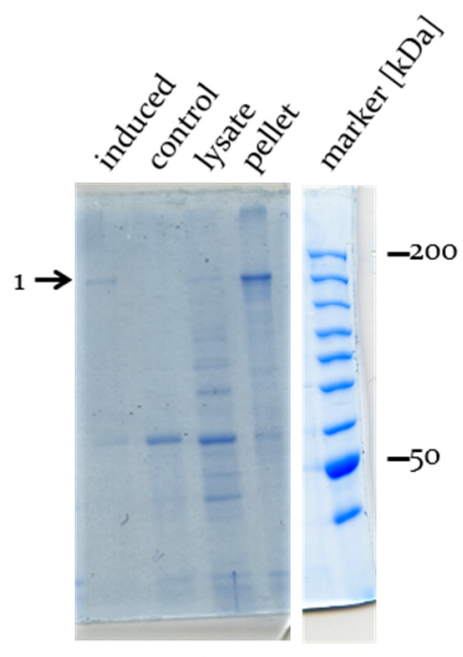

1 His $_{6}$-Slam-ZZ
Figure 11 Expression and solubility of recombinantly produced Slam

protein. The full-length protein (His ${ }_{6}-\mathrm{Slam}-\mathrm{ZZ}$ ) runs higher than its calculated weight (145 kDa) at approximately $160 \mathrm{kDa}$. The induced sample was IPTG-treated. The control sample was not induced with IPTG. A comparison of soluble (lysate) and insoluble (pellet) protein reveals a low solubility of full-length Slam.

Under 3.1.2.1 and 3.1.2.2 optimized example purifications are described to outline the progress in recombinant Slam purification, with the objective to facilitate future purification efforts.

\subsubsection{Slam $^{24-527}$ (N-Terminal)}

GST-tagged Slam ${ }^{24-527}$ is lacking the first 23 amino acids of the Slam sequence. Amino acid 164 to 532 have recently been found to be necessary and sufficient for cortical localization of Slam protein in S2 cells (Yan and Großhans 2018). Thus, the N-terminal third might carry a localization function for Slam. GST-Slam ${ }^{24-527}$ exhibits moderate solubility (figure 12), which was increased by choosing a richer growth medium for the expression host (figure 13). 


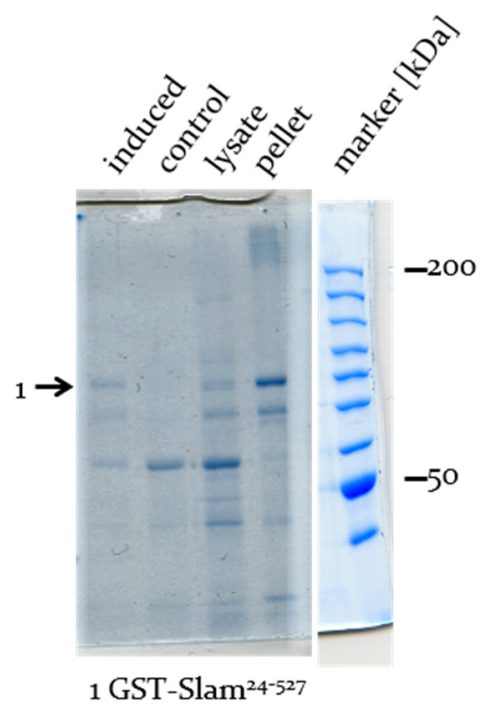

Figure 12 Expression and solubility of recombinantly produced truncated Slam.

GST-Slam ${ }^{24-527}$ shows a moderate solubility. The induced sample was IPTGtreated. The control sample was not induced with IPTG. A comparison of soluble (lysate) and insoluble (pellet) protein reveals a low solubility of Slams $N$-terminal third.

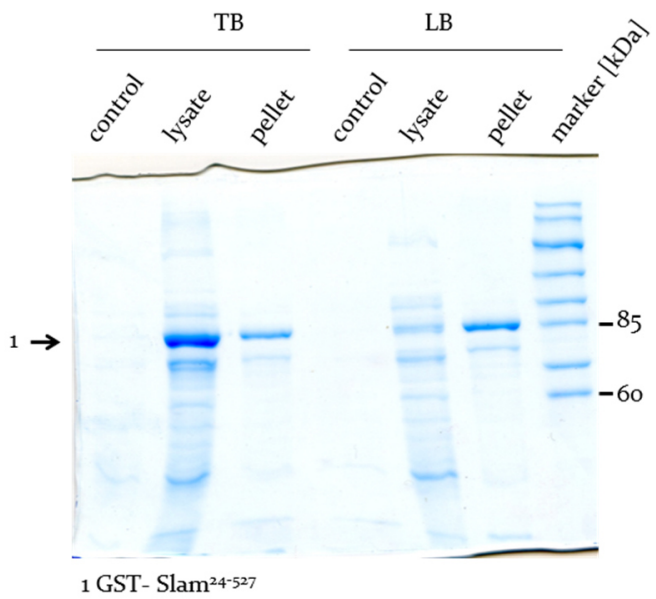

Figure 13 GST-Slam ${ }^{24-527}$ solubility is increased with changing growth medium for the expression host. When using TB-medium the quantity of protein in solution increases compared to LB-medium.

A one step affinity purification procedure (GSTrap 4B, GE healthcare) of GST-Slam ${ }^{24-527}$ from a $3 \mathrm{~L}$ culture results in approximately o,3 $\mathrm{mg}$ comparably clean protein (figure 14 ). Other visible protein bands are likely to be degradation products of GST-Slam ${ }^{24-527}$ since they appear only after the purification procedure and increase with time.

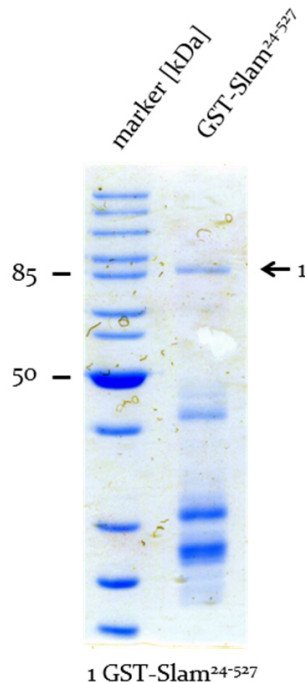

Figure 14 Purification of GST-Slam ${ }^{24-527}$. One chromatographic step (GSTrap 4B) removes most of the protein impurities from the lysate. Additional visible bands are likely to be degradation products of GST-Slam ${ }^{24-527}$. 


\subsubsection{Slam $^{1169-1196}$ (C-Terminal)}

GST- Slam 1069-1196

The C-terminal part of Slam was chosen for purification attempts for several reasons. The secondary structure prediction of the C-terminal end of Slam indicates a comparibly abundant structure (helices) and a low disorder profile (figure 8 and 9). The C-terminus of Slam has been found to be involved in its downstream signalling (Wenzl et al. 2010). Furthermore the Cterminal third of Slam protein seems to be essential for the regulation of the downstream effectors (Yan et al. 2017).

In figure 15 the purification plan for the GST (glutathione-S-transferase)-tagged C-terminal part of Slam is depicted. The strong affinity between GST and immobilized glutathione serves as capturing step. Subsequently the GST-tag is removed by PreScission protease (gifted from Prof. Jan Faix). The GST-tag and Slam ${ }^{1069-1196}$ are separated by another step of affinity chromatography. Since it was observed that those three steps do not yield a sufficient purity of Slam ${ }^{1069-1196}$, an anion exchange chromatography functions as an intermediate purification and the size exclusion chromatography as polishing step.

\begin{tabular}{|c|c|c|c|c|c|}
\hline $\begin{array}{c}\text { Affinity } \\
\text { Chromatography } \\
\text { (GSTrap 4B 5mL) }\end{array}$ & $\begin{array}{l}\text { PreScission } \\
\text { treatment }\end{array}$ & $\begin{array}{c}\text { Affinity } \\
\text { Chromatography } \\
\text { (GSTrap 4B 5mL) }\end{array}$ & & $\begin{array}{c}\text { Anion Exchange } \\
\text { (HiTrap Q HP } \\
1 \mathrm{~mL})\end{array}$ & $\begin{array}{c}\text { Gel Filtration } \\
\text { (HiLoad 16/600 } \\
\text { 200pg) }\end{array}$ \\
\hline
\end{tabular}

Figure 15 Overview of the multistep purification procedure of GST-Slam ${ }^{1069-1196,}$, which includes affinity, anion-exchange, and size-exclusion chromatographic steps.

In figure 16 the first step of the multistep purification procedure of Slam ${ }^{1069-1196}$ is shown. The cleared lysate was applied to a pre-equilibrated GSTrap 4B column (GE Healthcare). A major part of the protein bound to the column (B) and was eluted in 3 fractions (A,B) with reduced glutathione (50 mM Tris- $\mathrm{HCl}, 10 \mathrm{mM}$ reduced glutathione, $\mathrm{pH}$ 8.o). The purity of GST- Slam ${ }^{1069-}$ ${ }^{1196}$ was greatly increased by the first purification step. The 3 elution fractions, that contained GST- Slam ${ }^{1069-1196}$, were pooled and PreScission treated (C). PreScission protease removes the GST-tag from Slam ${ }^{1069-1196}$ and cuts 8 amino acids before the start of Slam ${ }^{1069-1196}$. It will therefore leave Slam ${ }^{1069-1196}$ with 8 additional amino acids. After protease treatment the buffer was changed to PBS and the protein mixture was reloaded to the GSTrap to separate tag and 


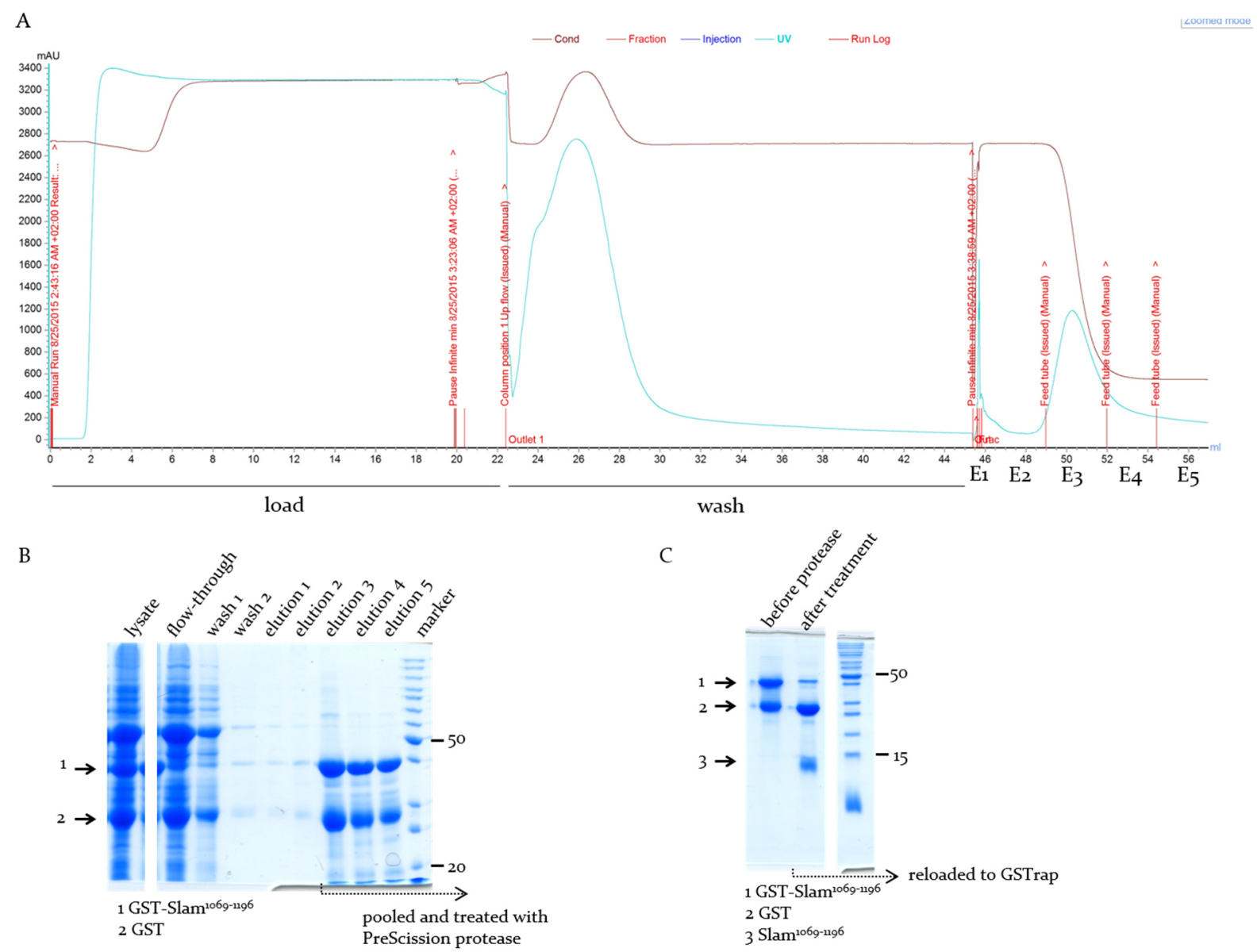

Figure 16 Capturing step of GST- Slam ${ }^{1069-1196}$ purification: GST-Slam ${ }^{1069-1196}$ was purified using a GSTrap. (A,B) The tag was removed using PreScission protease (C) and reloaded to the column to separate GST and Slam ${ }^{1069-1196}$. (A) Chromatogram of the first purification step

Slam ${ }^{1069-1196}$. Even though still detectable, large parts of the GST-tag in flow-through and wash were removed. Therefore, flow-through and wash fraction were pooled and underwent the next purification step (figure 17). The theoretical $\mathrm{pH}(\mathrm{I})$ of Slam ${ }^{1069-1196}$ computes to 4.82. Hence, an anion-exchange chromatography was chosen as next purification step. The buffer was changed to anion-exchange loading buffer (5o mM Tris, 5omM sodium chloride $\mathrm{pH} 7.5$ ) and the sample was applied to a pre-equilibrated strong anion exchanger (HiTrap Q HP, GE Healthcare) column. Bound protein was eluted (elution buffer $50 \mathrm{mM}$ Tris , 1 M sodium chloride $\mathrm{pH} 7.5$ ) by a linear gradient (see figure $18, \mathrm{~A}$ ). In figure 18 the chromatogram and analytics of the anionexchange chromatography step are depicted. In the chromatogram (A) the, mainly masked, peak of Slam ${ }^{1069-1196}$ was marked 1. Fraction 7 showed the strongest Slam ${ }^{1069-1196}$-band (B) and higher fractions contain decreasing amounts. This indicates that the peak marked 2 belongs to a different protein, though it still includes of Slam ${ }^{1069-1196}$. A doublet cannot be completely ruled out. Concerning the polishing success of C-terminal Slam during the IEX, especially in fraction 6 and 7 higher molecular weight proteins were separated from Slam ${ }^{1069-1196}$. Albeit the 
achievement of a small increase in purity for Slam ${ }^{1069-1196}$, there are still several impurities detectable (B). During the subsequent gel filtration (HiLoad 16/6oo Superdex 200 pg, GE Healthcare) of the pooled fractions 6 and 7, Slam ${ }^{1069-1196}$ and GST were still not completely separated (figure 19). Also, the amount of C-terminal Slam decreased significantly, even though still detectable by a Coomassie-stain after SDS-PAGE separation.

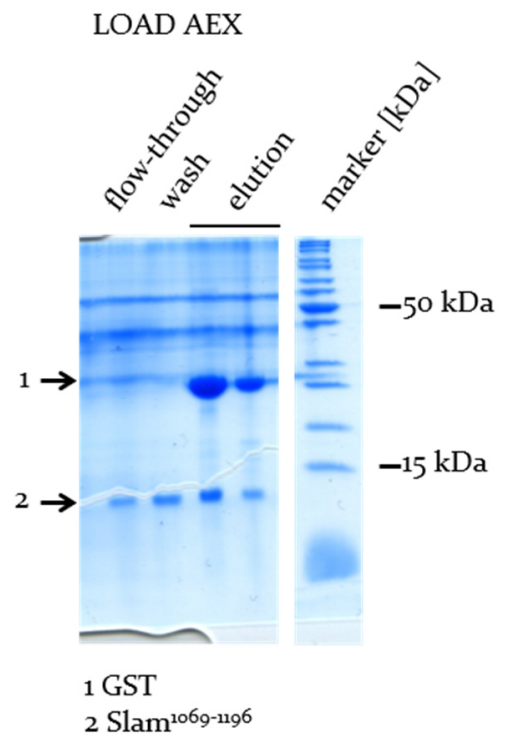

Figure 17 Reapplication of protease-treated protein mixture to the GSTrap $4 B$ to separate $\mathbf{t a g}$ and Slam ${ }^{1069-1196}$. The fractions labelled with 'LOAD AEX' are the samples, that were pooled (flowthrough\&wash) and underwent the next purification step (ionexchange chromatography) 
A

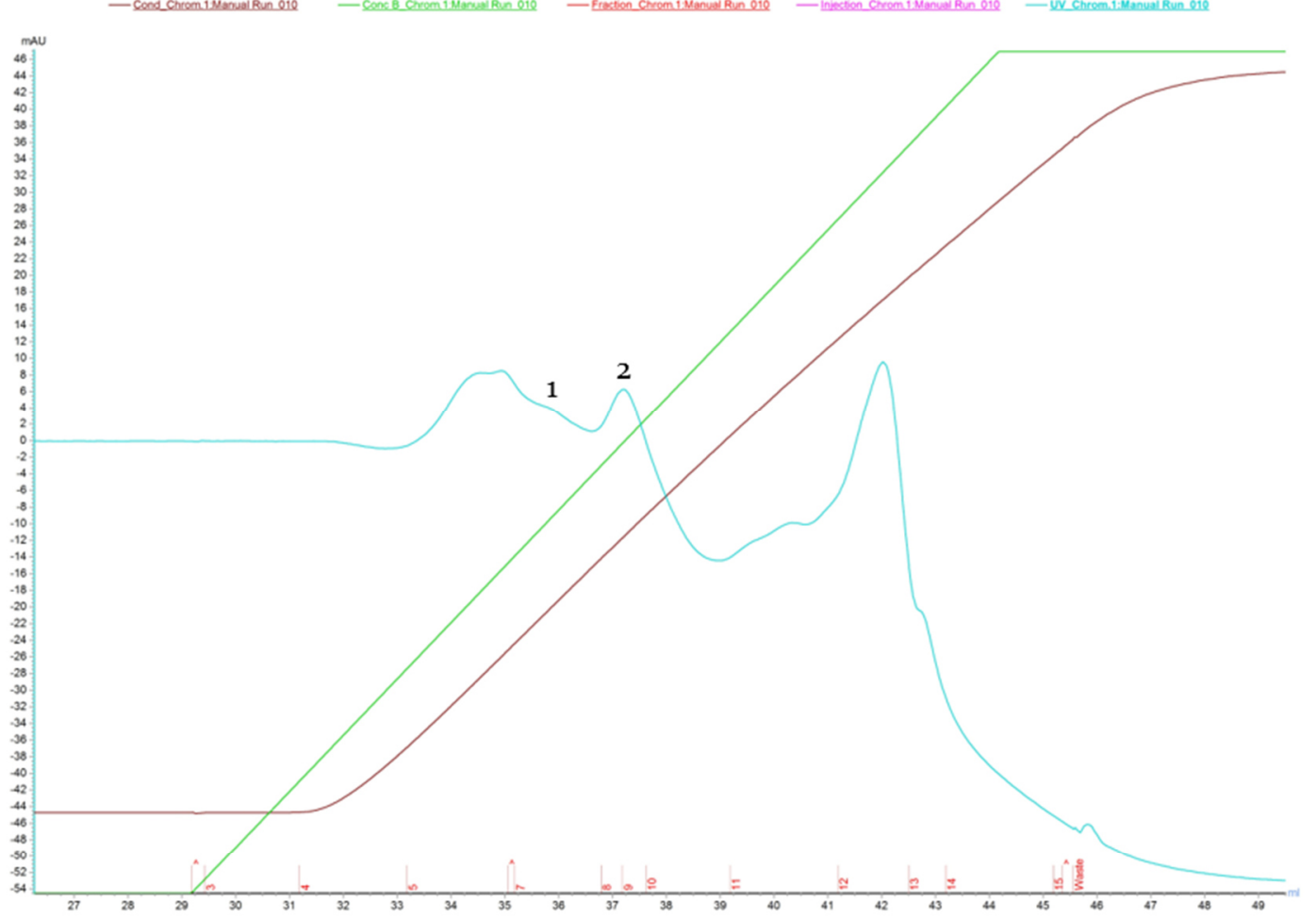

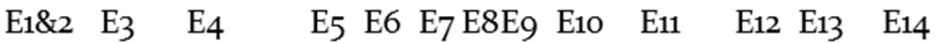

B

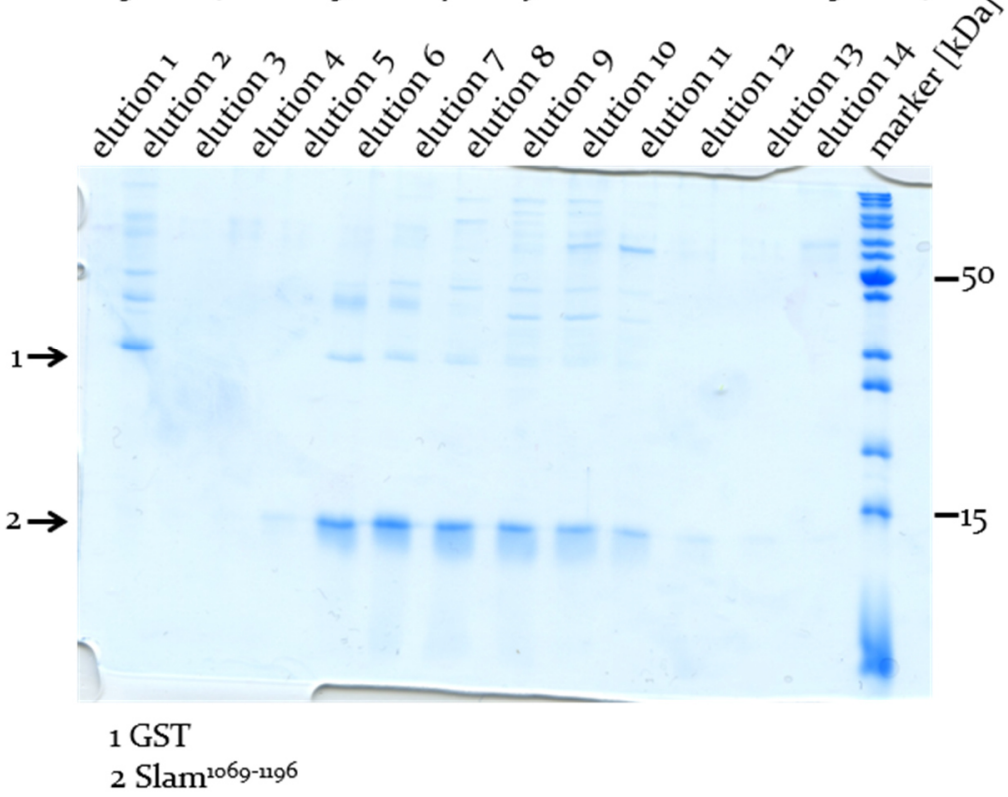

Figure 18 Anion-exchange chromatography of Slam ${ }^{1069-1196}$. The chromatogram (A) and stained SDS-gel (B) are depicted. The elution peak 1 can be ascribed to Slam ${ }^{1069-1196}$ (A) Analytics revealed several impurities left in the sample. (B) 
A

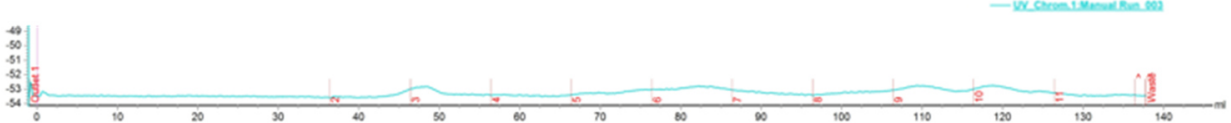

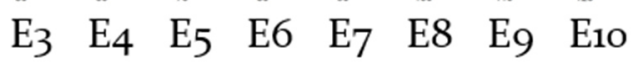

B

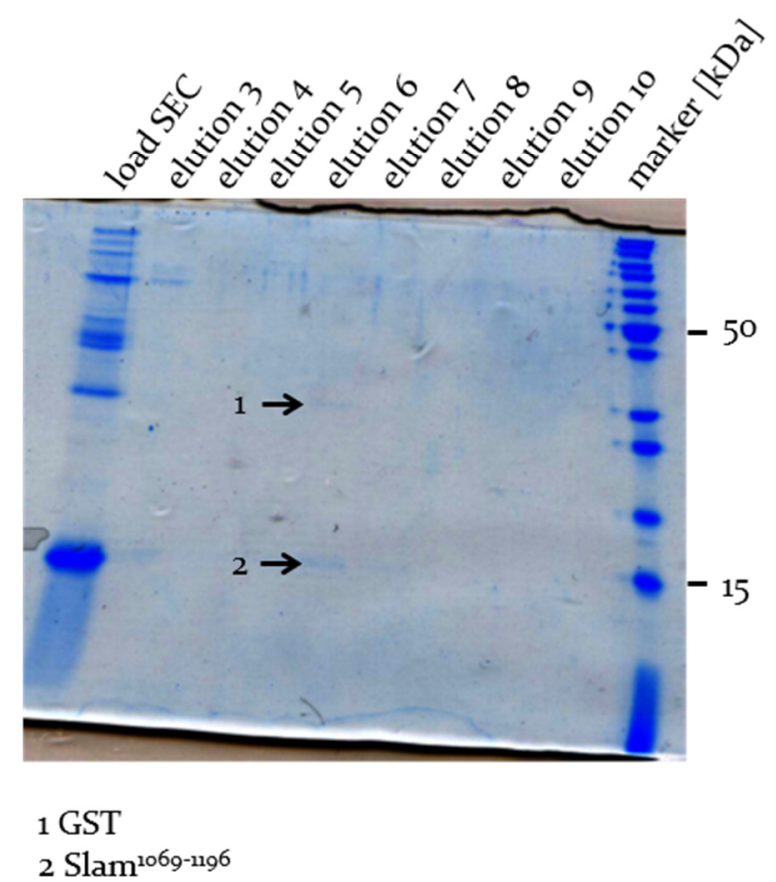

Figure 19 Last step of the multi-step purification of Slam ${ }^{1069-1196: ~ S i z e-e x c l u s i o n ~ c h r o m a t o g r a p h y . ~(A) ~ T h e ~ c h r o m a t o g r a m ~}$ indicates a strong decrease in protein concentration. (B) Slam ${ }^{1069-1196}$ is detectable by Coomassie-stain after SDS-PAGE, but still not pure enough for crystallization attempts.

Slam ${ }^{1069-1196}$ was recombinantly expressed and has been purified employing affinity, ion-exchange and size-exclusion chromatography. As, after strong optimization efforts, the C-terminus of Slam was still contaminated with impurities and did not meet the purity requirements to undergo crystallization attempts an additional His-tag was introduced and yet the possibility of another purification step.

\section{GST- Slam ${ }^{1069-1196-H i s 6}$}

To increase the purity of Slam ${ }^{1069-1196}$, a C-terminal hexahistidine tag was introduced by in-fusion cloning of slam(aa1o69-1196) to pGEX6OH (conducted by Lenin Chandran).

pGEX6OH codes for a N-terminal GST and C-terminal poly-histidine tag. This allowed another step of affinity purification and thus a new design of the Slam ${ }^{1069-1196}$ multistep purification procedure. The tag is small and there usually is no need to remove the tag. It binds strong and specific to immobilized metal ions during affinity chromatography (IMAC) at a neutral $\mathrm{pH}$. 
The solubility of GST-Slam ${ }^{1069-1196}$-His 6 appeared to be notably worse than the solubility of GSTSlam ${ }^{1069-1196}$ (figure 20). Concerning GST- Slam ${ }^{1069-1196}$, approximately half of the produced protein was found to be in the soluble fraction (supernatant, figure $20 \mathrm{~A}$ ). GST-Slam ${ }^{1069-1196}-\mathrm{His}_{6}$ seemed to be not soluble at all (figure $20 \mathrm{~B}$ ). Different $E$. coli host trains were tried, and expression media were altered (figure $20 \mathrm{~B}$ ). There was no gain in solubility of the His-tagged C-terminal Slam fragment. The theoretical PI of GST-Slam ${ }^{1069-1196}$ is lower, than the pI of GST-Slam ${ }^{1069-1196}-\mathrm{His}_{6}$, that is, 5.79 for GST-Slam ${ }^{1069-1196}$ and 6.57 for GST- Slam ${ }^{1069-1196}-$ His $_{6}$. In spite of the higher pI of GST-Slam ${ }^{1069-1196}-\mathrm{His}_{6}$ the $\mathrm{pH}$ difference of the standard lysis buffer and the pI should be big enough with 0,93. Regardless, a lysis buffer with a higher $\mathrm{pH}$ (9) and a slightly different lysis method (three instead of one freeze/thaw cycles before sonication) was tested (figure 21). The solubility increased but was still low.

A

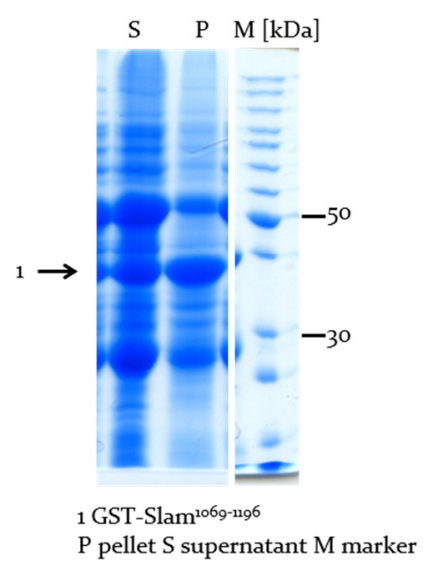

B

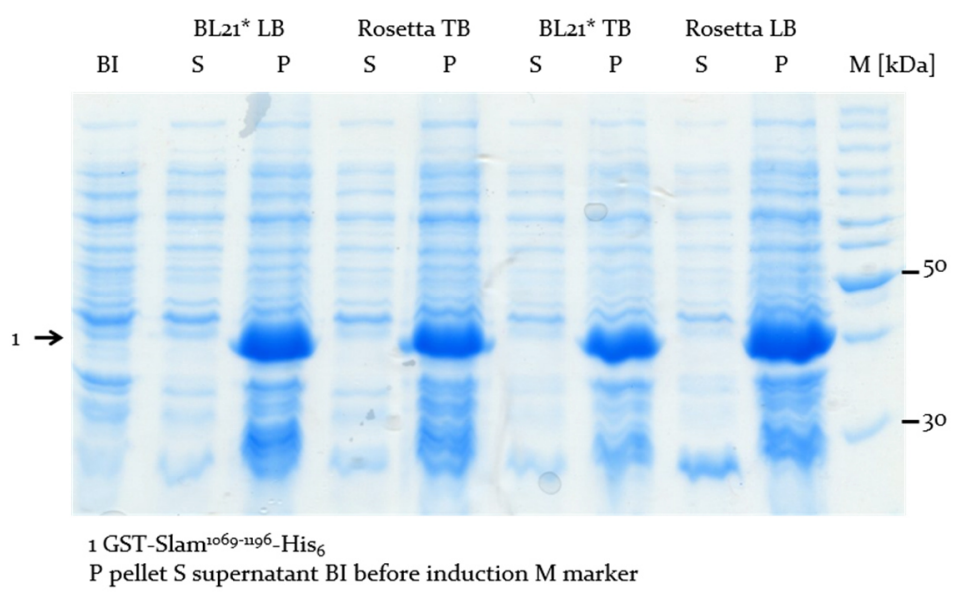

Figure 20 Analysis of GST-Slam ${ }^{1069-1196}$ and GST- Slam ${ }^{1069-1196-H i s}{ }_{6}$ solubility by examining protein content in supernatant and pellet after lysis via SDS-PAGE and Coomassie stain. The solubility of GST-Slam ${ }^{1069-1196-H i 6_{6}}$ is remarkably lower, than the solubility of GST-Slam ${ }^{1069-1196}$. Solubility of GST-Slam ${ }^{1069-1196}$ after one freeze/thaw cycle and sonication (A) Solubility screen of GST-Slam ${ }^{1069-1196-H i s_{6}}$ altering expression host and media (B) 


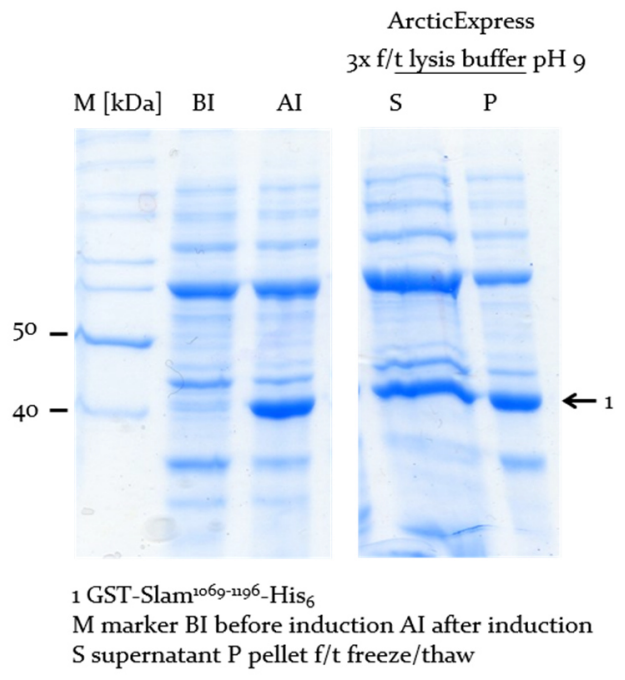

Figure 21 Solubility of GST-Slam ${ }^{1069-196-H i s_{6}}$ after three freeze/thaw cycles and a $\mathrm{pH}$ increase of lysis buffer

The hexahistinde tag was introduced to gain purity of Slam ${ }^{1069-1196}$ during a purification procedure. In figure 22 the two-step purification procedure for GST-Slam ${ }^{1069-1196}$-His 6 is depicted. Affinity chromatography serves as capturing (GSTrap) and as polishing (HisTrap, GE Healthcare) step. After the first purification step the GST-tag is proteolytically removed.

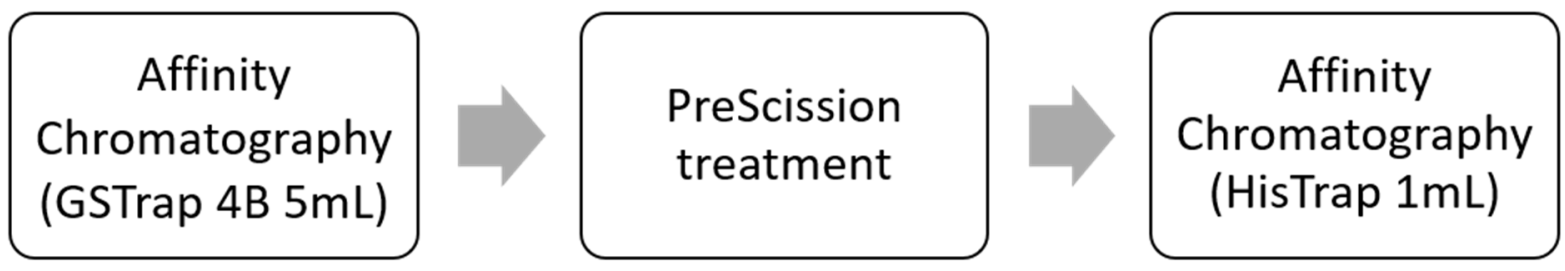

Figure 22 GST-Slam ${ }^{1069-1196-H i s_{6}}$ two-step purification plan.

For purification, $6 \mathrm{~g}$ frozen E. coli cells, that had produced GST-Slam ${ }^{1069-1196}-\mathrm{His}_{6}$, were thawed and lysed (standard lysis buffer $\mathrm{pH}$ 9; 3 freeze/thaw cycles). The cleared lysate was applied to a pre-equilibrated GSTrap $4 \mathrm{~B}(5 \mathrm{~mL}$, see figure $22 \mathrm{~A}$ and $\mathrm{B})$. Bound protein was eluted with reduced glutathione (50 mM Tris- $\mathrm{HCl}, 10 \mathrm{mM}$ reduced glutathione, $\mathrm{pH}$ 8.o). Fraction 4,5 and 7 were pooled and underwent an o/n PreScission protease treatment (see figure 23). The protein mixture was applied to a pre-equilibrated ( $20 \mathrm{mM}$ sodium phosphate, $500 \mathrm{mM}$ sodium chloride, 30 mM imidazole $\mathrm{pH}$ 7.5) HisTrap HP (1 mL) and bound protein gradient eluted (20 mM sodium phosphate, $500 \mathrm{mM}$ sodium chloride, $500 \mathrm{mM}$ imidazole $\mathrm{pH} 7 \cdot 5$ ). The analysis of the samples is shown in figure 24. The eluted protein got separated from most impurities. Albeit its bad solubility and the disadvantages that come with a low protein yield, the His-tag eased the purification procedure and increased the protein purity within a two-step purification procedure. 
A

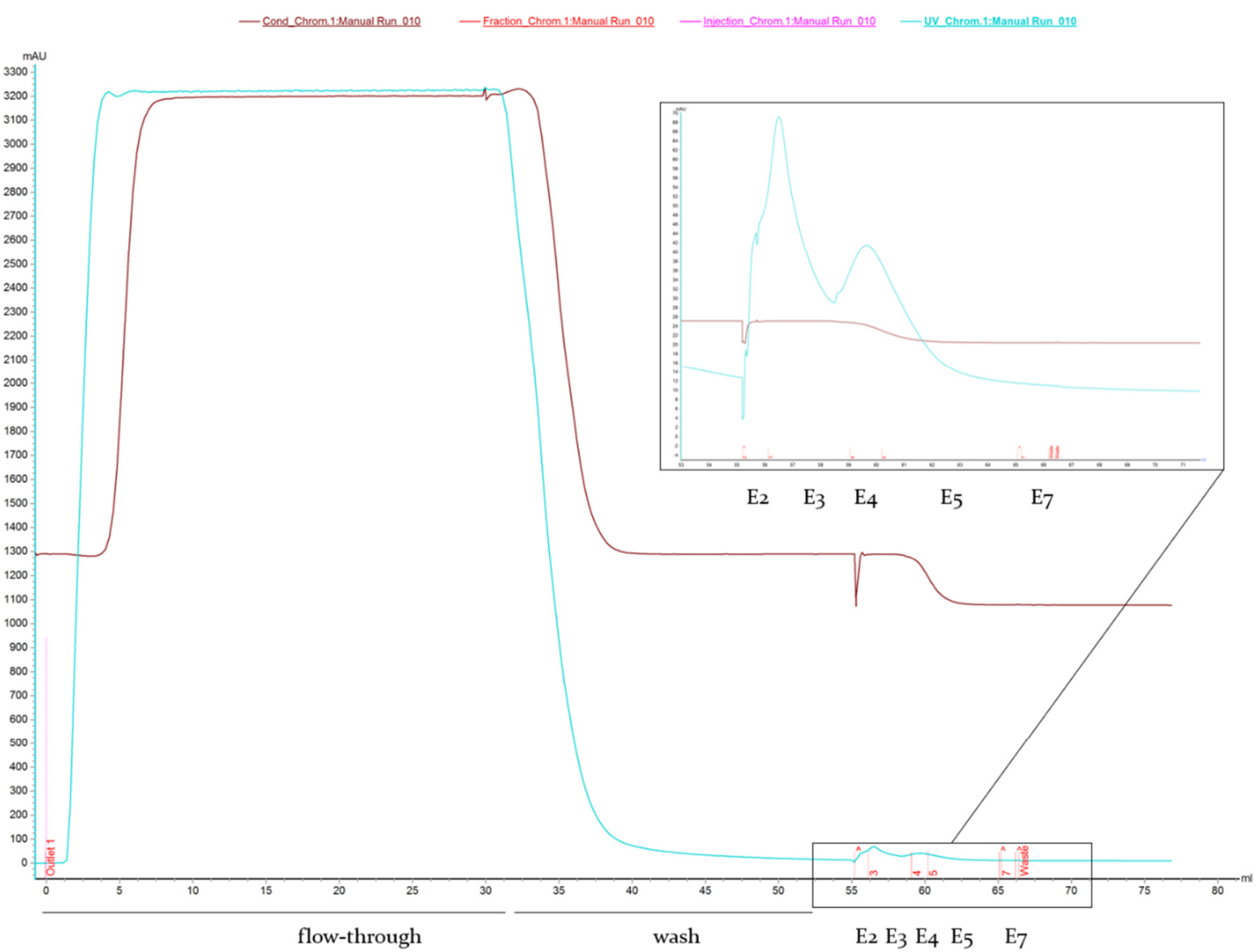

B

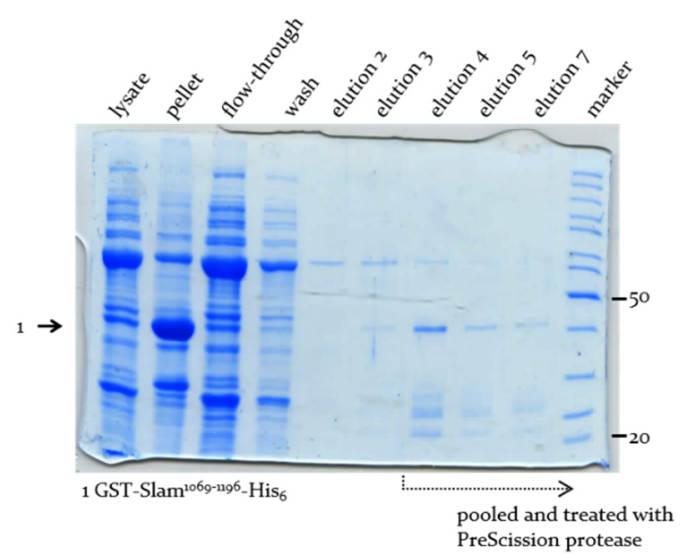

C

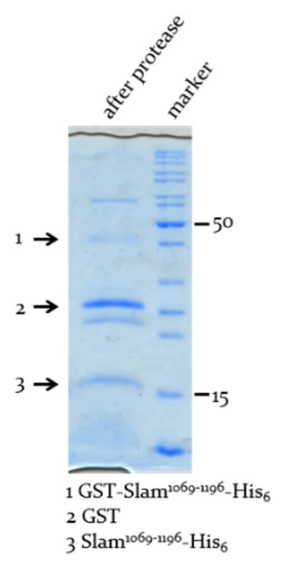

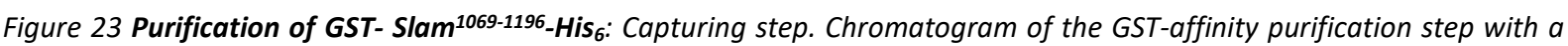
zoomed depiction of the elution. (A) Biochemical analysis via SDS-PAGE and Coomassie stain of samples. (B) Analysis of the proteolytic cleavage of the GST-tag from the rest of the protein. (C) 


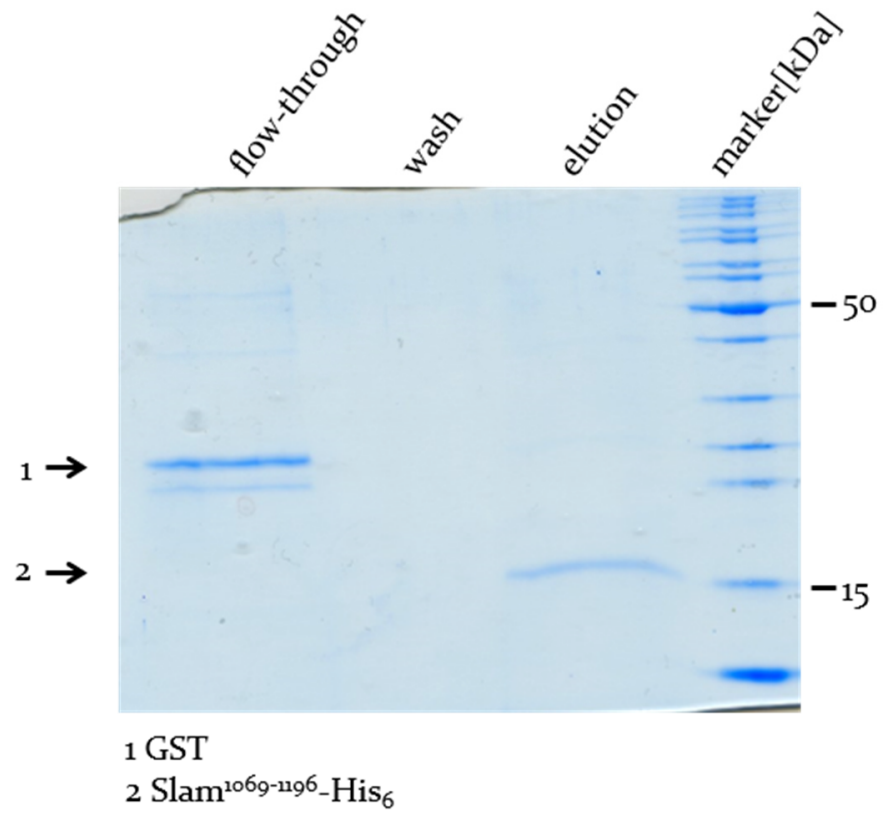

Figure 24 Purification of GST- Slam ${ }^{1069-1196}$ His6: Polishing step. Biochemical analysis of affinity (HisTrap) chromatographic samples to separate GST and left GST- Slam 1069-1196-His 6 from Slam ${ }^{1069-1196-H i s_{6} .}$ Most of the protein could be separated from Slam ${ }^{1069-1196-H i s_{6}}$.

\subsubsection{GST-His6 Slam truncations}

Slams structure is unknown and there are no bioinformatically recognizable domains over the span of its sequence. The full-length protein is vastly insoluble. Solubility is a prerequisite to most biochemical and structural approaches. The low solubility of Slam and the two beforementioned Slam truncations made it impossible to obtain sufficient amounts of protein for crystallization attempts. For a solubility screen, Slam N- and C-terminal truncations were constructed. The design was based on the secondary structure prediction (PSIPRED and DISOPRED $_{3}$ ) and the boundaries that potentially derive from it. For visualization RePROF (Rost and Sander 1993) and Meta-Disorder (A. Schlessinger, Yachdav, and Rost 2006; Avner Schlessinger et al. 2009) was employed. In figure 25 the created Slam truncations are depicted. Since intrinsic disorder may decrease the solubility of a heterologous produced protein, the truncations were designed around the middle part of Slam, that predicts to be completely unstructured. The plasmids are available for a solubility screen. 

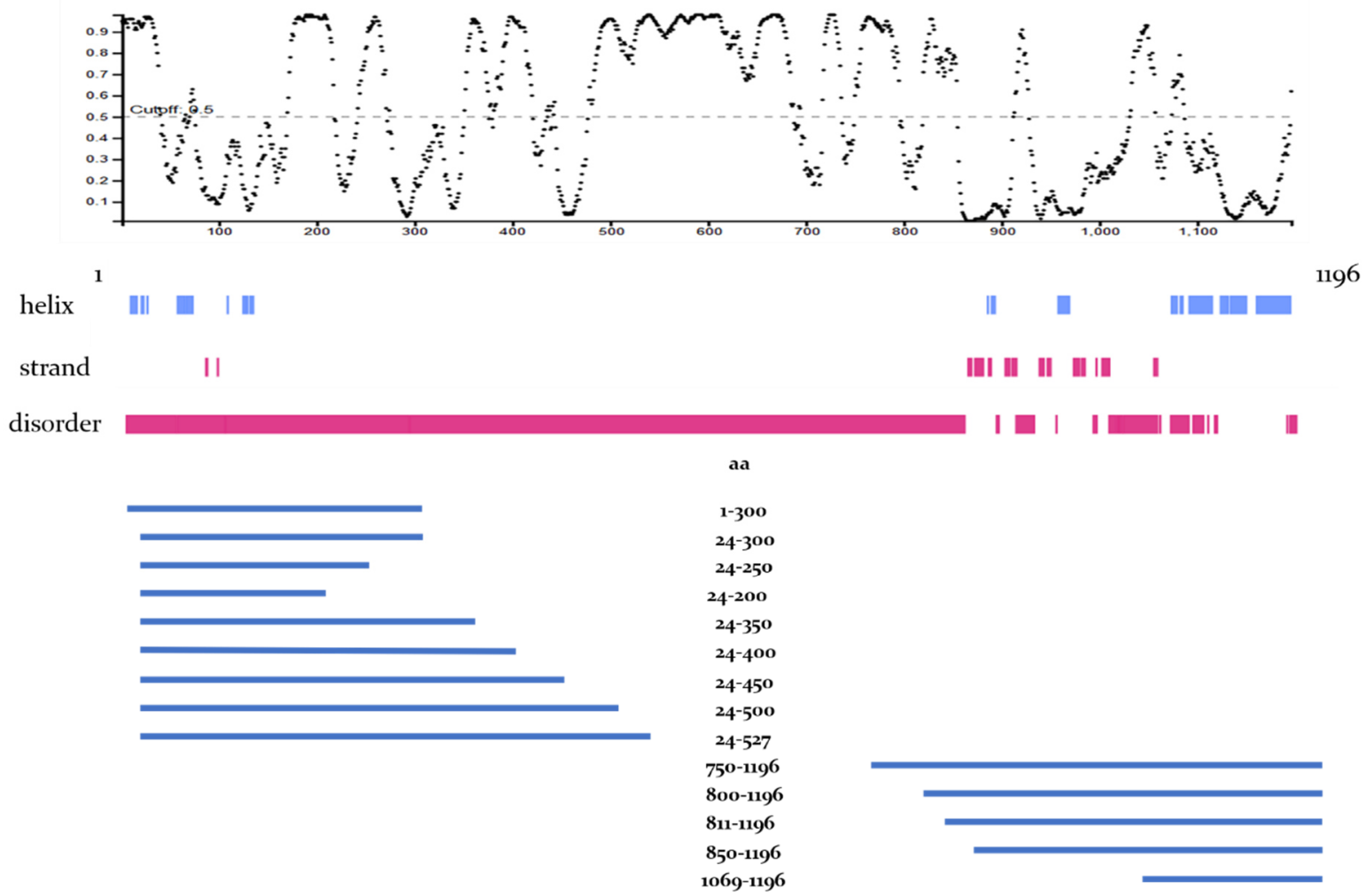

Figure 25 Slam truncations for a solubility screen. Depicted are the DISOPRED3 disorder prediction (upmost), the RePROF and Meta-Disorder prediction and the respective truncations, which have been cloned into $\mathrm{PGEX}-6 \mathrm{O}-\mathrm{H}$ to be $\mathrm{N}$-terminally GST-and C-terminally His 6 -tagged

\subsubsection{Final conclusions}

Slam truncations were recombinantly produced and purification procedures suggested. The fulllength protein and the N-terminal third are rather unsuitable for production and purification of protein aiming mg amounts. This is due to their low solubility and stability.

GST-tagged C-terminal 129aa of Slam displayed higher stability and good solubility. The disadvantage of a GST-tagged truncation was the purification procedure itself. It was not possible to separate all impurities from the protein of interest. Including impurities, it was possible to gain several mg amounts of protein from a $2 \mathrm{~L}$ culture in a two-step purification procedure.

A C-terminal His-tag decreased the solubility but made it possible to gain sufficiently clean protein after a two-step purification. The low solubility would make a very high culture volume necessary to obtain several mg amounts of protein for crystallization attempts. It is not clear, however, if the obtained protein has the qualities for good crystallization.

Several N-terminal and C-terminal GST-His 6 Slam truncations were constructed but first solubility screens seemed unpropitious. 
My conclusive suggestion is to focus on functional assays in the future since Slam purification is intricate and the chances to gain usable crystals and diffraction data is in my opinion too low.

\subsection{Interactome of Slam}

\subsubsection{Putative interactors in data bases and previous reports}

Some interactors of Slam have previously been described. During cellularization Slam is necessary to recruit the Rho-activating guanyl-nucleotide exchange factor RhoGEF2 to the furrow by its PDZ domain (Grosshans et al. 2005; Wenzl et al. 2010). RhoGEF2 in turn is needed to localize the formin Diaphanous (Dia) to the furrow. RhoGEF2 and the Dia are both necessary for furrow formation (Grosshans et al. 2005). Both share the same initial localization with Slam at the tip of the emerging furrow. RhoGEF2 positively regulates Rho1 during cellularisation, which also localizes to the FC and regulates Dia and Myosin II (zipper) (Barrett, Leptin, and Settleman 1997; Grosshans et al. 2005, o; Acharya 2014). Dia nucleates actin filaments. Myosin II is a non-muscle myosin that drives actomyosin contraction. Myosin II localization is also dependent on and is even ectopically recruited by Slam (Thomas Lecuit, Samanta, and Wieschaus 2002).

A direct interaction has not only been shown for RhoGEF2 but also Patj, which again perfectly colocalizes with Slam at the furrow and basal particles (Wenzl et al. 2010). Patj was reported to play supporting roles in the stabilisation of adherens junctions by interacting with the Myosinbinding subunit of Myosin phosphatase, subsequently decreasing Myosin dephosphorylation, which again results in more activated Myosin (Sen, Nagy-Zsvér-Vadas, and Krahn 2012). Patj has 4 PDZ domains and the interaction to Slam seems to be mediated by the C-terminus of Slam, since a C-terminal truncation (slam ${ }^{\text {waldos }}$ ) does clearly reduce Patj levels at the FC (Wenzl et al. 2010).

A direct interaction has also been described for Slam and Oskar (Hurd et al. 2016). Apart from the similarity that oskar mRNA is also spatiotemporally controlled, Slam and Oskar have no known intersections and complete different localizations (Krauss et al. 2009). 
During cellularization there is cytosolic- and membrane-attached Slam. Slam is associated to the plasma membrane without being a transmembrane protein. It can be detached from the membrane by using high salt (fig. 26)(Acharya 2014). For initial immunoprecipitations (IP) the buffer of choice was RIPA buffer, which contains ionic detergents (SDS and sodium deoxycholate) and disrupts membranes. Lysis with RIPA buffer will yield a higher amount of membrane attached Slam, but also tends to disrupt protein complexes, and was therefore replaced with a milder IP buffer that solubilizes some of the membrane-attached, but mainly cytosolic Slam (fractionation assays, not shown). During co-IP experiments with Slam and its mRNA Dr. Shuling Yan found that reduced salt and detergent concentrations in RIPA buffer will preserve the membrane attached complex, that contains Slam and its RNA. The use of RIPA(-) (75 mM salt and no SDS) however, will still have a comparatively high yield of Slam. The fact, that the IP buffer solubilizes less of the membrane attached Slam gives the opportunity of comparison. Will varying buffer conditions give different results during mass spectrometric analysis and therefore indicate distinct cytosolic and membrane attached interaction partners?

For this study flies were used that carry N-terminally GFP-tagged Slam. Flies that carry only GFPSlam and no endogenous Slam are not viable. They are able to cellularize but will not complete development. Additionally GFPSlam localizes less to the membrane than wild type Slam (fig.26) (Acharya 2014). For the following study it must be kept in mind that GFP seems to interfere with the function of Slam to some degree. 


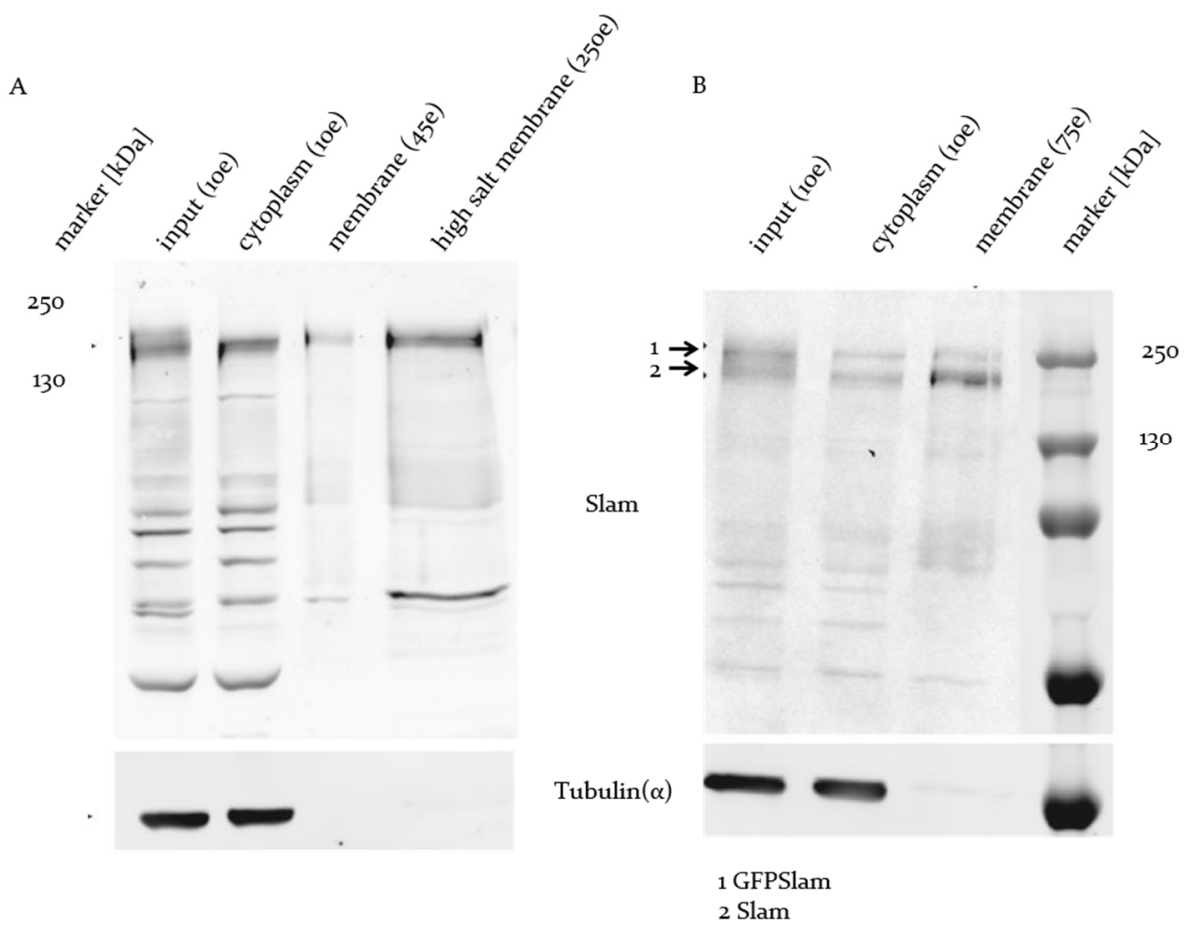

Figure 26 Slam is associated to membranes. (modified from (Acharya 2014)) Slam is no transmembrane protein as seen by the solubilization of Slam with high salt (1M NaCl). Cellularizing embryos have been lysed in fractionation buffer (50 mM Tris pH 7.5, $75 \mathrm{mM} \mathrm{NaCl} 1 \mathrm{mM} \mathrm{MgCl}$, 0.05\% NP-40, 1 mM DTT, $2 \mathrm{mM} \mathrm{PMSF,} 1$ X Roche Protease inhibitor cocktail, $0.01 \mathrm{U} / \mu \mathrm{L}$ RNase inhibitor). A sample was taken. After centrifugation a sample from the supernatant (cytoplasm) and the pellet (membrane) was taken. The residual pellet was redissolved in fractionation buffer and $1 \mathrm{M} \mathrm{NaCl}$ was added. After centrifugation a sample from the supernatant was taken (high salt membrane).(A) During a fractionation experiment of staged (1.5-2.5h) GFPSlam mutant embryos it can be noted that GFPSlam has a lower affinity to the plasma membrane than wild-type Slam.(B) Both experiments were accomplished by Dr. Sreemukta Acharya. For detailed information please refer to her PhD thesis (Acharya 2014).

The conducted assay is a combination of an immunoprecipitation (use of antibody) and a pulldown assay (use of other affinity system but antibody). The underlying principle is the affinity of a) Streptavidin towards Biotin (pull-down) and b) of the biotinylated GFPbinder (anti-GFP nanobody) towards GFP (IP, figure 27). A biotinylated anti-GFP nanobinder (GFPbinder) was added to lysates of cellularizing embryos expressing transgenic GFPSlam. As a negative control a wild type lysate was treated equally. After the incubation time magnetic, Streptavidin-coupled beads (Invitrogen) were added to the solution. After binding time on a rotator, the beads were washed and subsequently treated with SUMOstar protease. SUMOstar cuts right at the end of the GFPbinder and selectively elutes bound protein and therefore putative binding partners of Slam into the solution. Ideally no wild-type protein binds to the beads. In figure 27 the basis (A) and an exemplified pull-down (B) of the conducted IP is depicted. The shown example IP underwent mass spectrometric analysis and evaluation (IP sample C). GFPSlam was bound (27B 
left) and elution functional, which strongly increases sample purity. WT Slam did not bind to the biotinylated GFPbinder nor the beads (27B right), hence represents a good negative control. Not every negative control, however, was completely devoid of Slam, which is visible in the mass spectrometric results. The eluted protein was acetone precipitated (GFPSlam as well as WT sample) and resuspended in the respective buffer and $2 x$ Laemmli. It was submitted to mass spectrometric analysis to the Scientific Core Facility Proteomics (CF Pro) of the University Medical Center Göttingen (Dr. Christof Lenz).

A
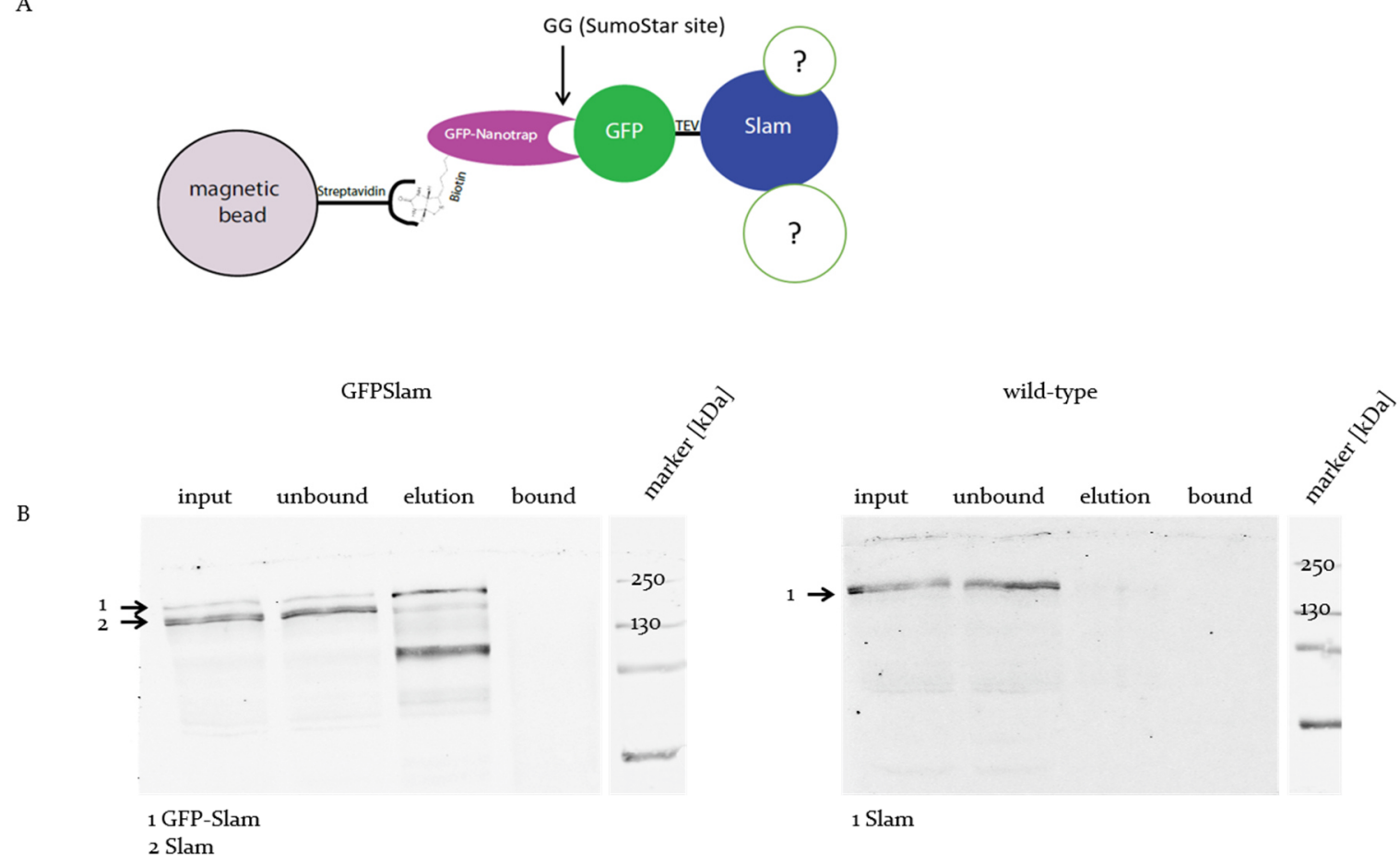

\begin{tabular}{|l|l|l|l|l|}
\hline sample applied (in ee) & input & unbound & elution & bound \\
\hline GFPSlam & 50 & 50 & 3720 & 3720 \\
\hline wild-type & 50 & 50 & 3500 & 3500 \\
\hline
\end{tabular}

Figure 27 GFPSIam pull-down assay for mass spectrometric analysis of putative Slam binding partners. (A) Simplified schematic of the GFPSlam pull-down assay. A biotinylated anti-GFP nanobody (GFPbinder) binds to GFPSlam and putative binding partners. Paramagnetic beads, that have Streptavidin covalently coupled to their surface, pull down the prebound complex of GFPbinder and Slam and its potential binding partners. SUMOstar protease cleaves between the nanotrap and Slam and leaves the Slam complex in solution and the nanotrap bound to the beads. The eluted proteins were acetone precipitated and submitted to mass spectrometric analysis. Samples of lysates from embryos expressing GFPSlam (genomic transgene) were compared to wild-type extracts. (B) Western blot analysis of samples that were submitted to mass spectrometry. The amount of embryo equivalents (ee) that were applied to the SDS-gel are indicated in the table below. GFPSlam was bound (right) and bound protein eluted by SUMOstar protease. The wild-type protein was not detectable by western blot in the eluted and bound samples (left). 


\subsubsection{IP-MS using immunoprecipitation (IP) buffer and detergent-containing (RIPA(-)) buffer}

Table 7 summarizes the mass spectrometric results. For detailed information and $p$-values please refer to the appendix.

\section{IP buffer samples}

The bait, Slam, was highly enriched in all 3 IP samples, albeit the spectral counts in sample A (1008) were $\sim 5$ times higher, than in sample B (209) and C (243). In sample A and C Slam was not detectable in the wild-type control samples. Sample B measured very little Slam in the wildtype control (one spectral count in one technical replicate). This indicates specificity of the immunoprecipitation/pulldown assay and plausibility of the detected binding partners.

Several proteins were found enriched in all 3 samples including the cell polarity protein Patj, the cytoplasmatic linker Restin homolog, the probable ubiquitin-protein ligase Herc 2 and its neuralized homologue and substrate $\mathrm{Neurl}_{4}$ (Al-Hakim et al. 2012).

The Drosophila CIN85/CD2AP orthologue Cindr interacts with Anillin (scraps) to control cytokinesis (Anillin has been measured with an averaged 4 SC in the mutant A sample but not in the WT or other samples). They colocalize at cleavage furrows, intercellular bridges and midbodies in dividing Drosophila S2 cells (Haglund et al. 2010). It was found enriched in 2 of the 3 samples isolated under IP buffer conditions (A and B). Low spectral counting shows high variability (Lundgren et al. 2010). Cindr may have fallen below the detection limit in sample $C$, since it was hardly measurable in sample B (averaged spectral counts (SC) of Slam 209 and Cindr 2) Sample C had only little more Slam measured ( Slam SC 243), therefore the overall protein quantity may have been similar. Taking the high variability in low spectral counting into account Cindr may very well have been masked during analysis of sample $C$.

Bottleneck, a key regulator of microfilament network during Drosophila cellularization, is worth mentioning for similar reasons. 33-fold increased total spectrum counts for Bottleneck were detected in IP sample A. However, no spectrum could be matched to Bottleneck in sample B and C. Bottleneck may have fallen below the detection limit in sample B and C, so it cannot be ruled out as an interactor of Slam of either direct or indirect kind.

Several actin-related proteins were enriched in one or more of the IP samples. Actin ${ }_{5} \mathrm{C}$ was detected 4-fold (ratio GFPSlam and wild type) in sample A. In sample B and C, however, Actin 57B was detected. In sample B it was slightly higher in GFPSlam embryos than wild type (FE 1.3). In sample $C$ actin was not detectable under the used settings (protein threshold $99 \%$, min. peptides 2, peptide threshold 95\%). Under changed settings (protein threshold $1 \%$ FDR, min. peptides 2, peptide threshold $1 \%$ FDR) though it was detectable but higher in the wild type 
control. Other actin related proteins behaved similar. The following proteins were only detected in sample A: Spectrin alpha chain (FE 24), the barbed end binding F-actin-capping protein subunit alpha and beta (FE 9) and parts of the actin polymerization regulating Arp 2/3 complex Arpc4 and Arpc5 (FE 9 and 13 respectively). In sample A and B alpha-actinin (FE 11 and only detected in GFPSlam sample with SC 9), Arpc1 (FE 5 and 3 ) and $\operatorname{Arp}_{3}$ (FE 13 and 3 ) were enriched compared to the wild type control. Arpc2 and Arp2 were enriched in sample A (FE 8 and only detected in GFPSlam sample with SC 7) but were also detectable in sample B (GFPSlam only) under the beforementioned changed setting (see also table 10 to 15 , appendix). Sample $C$ had less actin measured in the GFPSlam sample than the wild-type control. Correspondingly other actin related proteins were measured: Arpc2 and Arp2 were higher in the wild type sample. Arp3 was detected in very similar amounts in both samples: bait and control (averaged SC 12 and 11, respectively). To summarize (please consult table 7), several actin-binding and regulating proteins have clearly been detected and enriched in sample 1 (all of the above mentioned). In sample B proteins were either enriched in the GFPSlam sample, were below the detection limit or were not traceable. Sample C, however, had only little of the proteins detected (Act57B, Aprpc2/Arp2/Arp3) and except for Arp3, which has similar SC for both GFPSlam and wild-type control, they all were higher in the control. Since all the abovementioned actin-binding proteins are associated, it is no surprise, that their quantities correlate in one sample.

Another detected protein category are Myosins. In sample A the heavy chain of the unconventional myosin 95F (Jaguar) was 50-fold higher in the GFPSlam sample than the wildtype control. In sample B it has a 29 -fold enrichment and in sample $C$ it was slightly higher in the control. This result somehow matches the upper observation. The class VI unconventional myosin Jaguar transports particles along actin filaments during Drosophila syncytial blastoderm stage (Valerie Mermall, McNally, and Miller 1994). The blastoderm stage precedes cellularization. But also during cellularization Jaguar binds F-actin as shown by co-IP experiments (Sisson et al. 2000). Thus, Jaguar is interconnected to Actin. Since in sample C actin was higher in the control, it may interrelate with the slight enrichment of Jaguar in the latter. Myosin V (Dilute class unconventional myosin, didum) was detected in the A and B mutant sample but not in the control. It was not measurable in sample C. MyoV interestingly promotes posterior localization of Oskar mRNA in Drosophila oocytes by associating with Oskar mRNA transport complex and counterbalancing the microtubule-based motor Kinesin (Krauss et al. 2009). We do not know how Slam mRNA is transported from the nucleus to the tip of the invaginating furrow and maintained at the site of action. MyoV may play a role here. Further it should be noted that Kinesin light chain was detected in sample A (FE 2,5) but not in sample B 
or C. Kinesin heavy chain was identified in sample B $(\mathrm{FE} 4,6)$ and in sample C (slight enrichment in mutant sample FE 1,1).

Myosin VIIa, which is involved in cellular protrusion formation and cargo intracellular transport, was found in sample A and C (FE 18 and only detected in mutant sample with SC 6, respectively)(Yang et al. 2006; Daniel P Kiehart et al. 2004).

Non-muscle Myosin II (zipper) can bind and cross-link actin. It plays a crucial role during cellularization forming the actomyosin contractile apparatus together with F-actin (Miller and Kiehart 1995). In vivo Slam and MyoII colocalize at the intercap region and, later, at the most basal part of the furrow canal. Slam even appears to recruit MyoII to the furrow canal. (Thomas Lecuit, Samanta, and Wieschaus 2002) Accordingly, Myosin II heavy chain has been identified in sample A and C (FE 10 and 2, respectively). It was not detectable in sample B.

\section{RIPA(-) buffer samples}

In contrast to IP buffer, RIPA(-) buffer contains the biological and mild detergent deoxycholate and the non-ionic surfactant (detergent) Triton-X-10o. To preserve the Slam RNP SDS has been removed from the original RIPA recipe and salt has been reduced to $75 \mathrm{mM} \mathrm{NaCl}$. Despite the changes, RIPA(-) buffer seems to have dissolved some of the protein-protein interactions. The results are summarized in table 7 . For detailed information on the technical replicates and $p$ values please refer to table 10 to 15 in the appendix.

Slam was found enriched in all 3 biological replicates (FE 6, 4 and 418 for samples A, B and C, respectively). Sample C contained considerably more Slam than sample A and B. Patj, too, was found enriched in all 3 sampled (FE 7 and 5 for sample A and B, 314 SC for C but not detected in WT). Restin homolog, however, was only measured negligibly higher in the mutant sample of biol. replicate A and B (FE 1.08 and 1.05) and in sample C even higher in WT (not detected in GFPSlam). The ubiquitin-protein ligase Herc2 was higher in WT for sample A and B (FE o.8 and o.6) and not detected in sample C. Neurl 4 was detected in 2 samples at very low spectral counts (data not shown). Cindr again was detected in sample $A$ and $C$ in the mutant but not in the wildtype sample (SC 1 and 23). It was not identified in sample B.

Bottleneck was detected with a few SC in sample B but higher in WT (averaged SC 1 for mutant and 2 for WT).

In biological replicate A, Actin (FE 1.1), alpha Spectrin (FE 1.02), alpha Actinin (FE 1.1) , F-actincapping protein subunit alpha and beta (FE 1.2 and 1.3, respectively), Arpc1, 2 and 5 (all 3 FE 1.1) and Arp2 (FE 1.5) were all slightly higher in the bait-containing mutant sample, which again 
emphasizes their connection. Arpc4 was detected with the same SC in mutant and WT sample (FE 1). Arp3 was faintly higher in the WT sample (FE 0.97).

In RIPA(-) buffer replicate B the majority of the identified actin-related proteins measured slightly higher in the wild type sample. Actin (FE o.8), alpha Spectrin (FE 1.1), alpha Actinin (FE o.96) , F-actin-capping protein subunit alpha and beta (FE 1.2 and o.6, respectively), Arpcı (FE o.7), Arpc2 and 4 (FE o.8 ) and Arpc5 (FE o.7), Arp2 (FE o.8) and Arp3 (FE 1.06).

In replicate $C$ all actinin-related proteins were found higher in the wild type control. Except Arpc4 and 5, which were not detected at all. For fold enrichment values please refer to table 7 . Again, the variability of low spectral counting must be stressed here. It cannot be concluded that a protein is more abundant in a sample when the enrichment is based on low spectral counting. The results for this thesis were evaluated carefully and low spectral counts were only considered when they support distinct results from other replicates or for comparison. For itemized spectral counting please refer to the appendix. Also, the term fold enrichment might be misleading when the ratio of GFPSlam and WT falls under 1. For simplicity the term was maintained.

Jaguar was higher in WT for sample A and C (FE o.8 and o.1). In sample B it was minimally higher in the bait-containing sample (FE 1.04). MyoV was found enriched in the WT sample (A and B) and not detected in sample C. MyoVIIa was 2-fold enriched in sample A, more abundant in the WT in sample B (FE o.9) and not detected in sample C. MyoII was higher in WT for sample A and C (FE 0.7 and 0.4 ) and slightly more abundant in the mutant sample in biol. replicate B (FE $1.2)$.

Taken together, the RIPA(-) buffer samples gave very different results upon mass spectrometric analysis than the IP buffer treated embryos. Proteins that were distinctly enriched under milder lysis conditions gave random results under harsher conditions. One example is Restin homolog that was clearly more abundant in the bait-containing samples, when lysed with IP buffer, but was only insignificantly higher in embryos carrying GFPSlam under RIPA(-) lysis conditions. In case of RIPA(-) buffer replicate $C$ it even gave the opposite result. Restin homolog was not detected in the GFPSlam sample but in WT. The same applies to Herc2. Bottleneck too resulted in the opposite: higher in WT in one case, and undetectable in the other two. Under IP buffer conditions it was clearly enriched in one GFPSlam sample and undetectable in the other 2 replicates. Also, most of the Myosins yielded opposite results.

To summarize, there are only 2 proteins, that have been enriched in correlation with the bait under milder and harsher conditions: Patj and Cindr. The rest gave distinct results, when cellularizing embryos were lysed with IP buffer but more random results when lysed with detergents. This may hint towards a stronger (direct) association of Patj and Cindr with Slam. It 
seems likely, that under RIPA(-) buffer conditions the complex of Slam and its mRNA stays intact (Dr. Shuling Yan). 
Table 7 Selection of proteins identified in IP and Ripa(-) buffer samples by mass spectrometric analysis (bait: GFPSlam). Indicated are protein names, UniProt reference numbers, normalized total spectrum counts of GFPSlam (2 technical replicates have been averaged), fold enrichment (FE) towards wild-type sample and statistical significance (SS). The upper table presents the results of IP buffer-based samples and the lower table the samples, when RIPA(-) buffer was employed. For p-values and detailed information may be referred to the appendix. ndiWT not detected in wild type, nd not detected

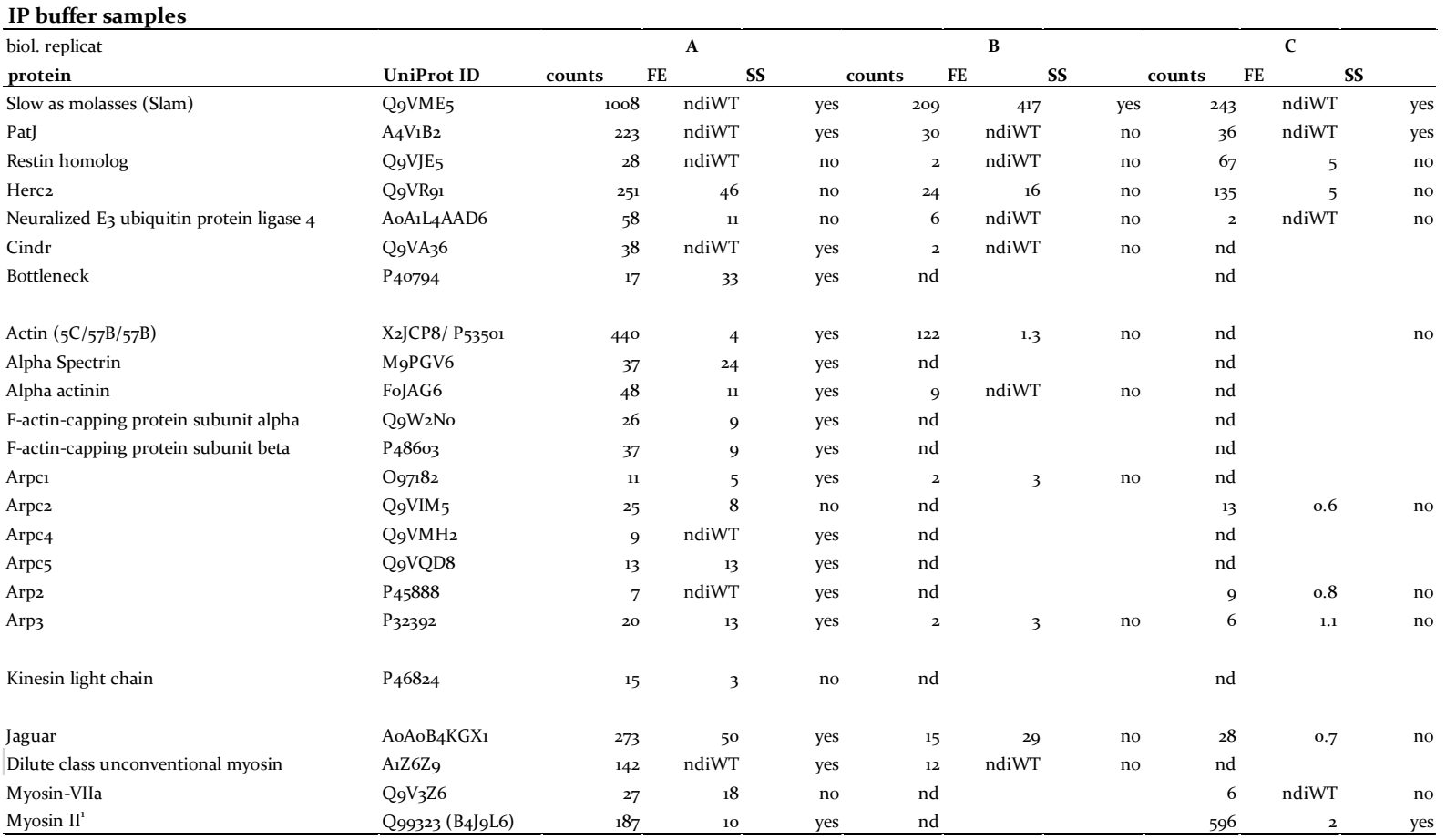

RIPA(-) buffer samples

\begin{tabular}{|c|c|c|c|c|c|c|c|c|c|c|}
\hline \multirow{2}{*}{$\begin{array}{l}\text { biol. replicat } \\
\text { protein }\end{array}$} & \multirow[b]{2}{*}{ UniProt ID } & \multicolumn{3}{|c|}{ A } & \multicolumn{2}{|c|}{ B } & \multirow[b]{2}{*}{ SS } & \multicolumn{3}{|c|}{ C } \\
\hline & & counts & FE & SS & counts & FE & & counts & FE & SS \\
\hline Slow as molasses (Slam) & $\mathrm{Q}_{9} \mathrm{VME}_{5}$ & 75 & 6 & yes & 96 & 4 & yes & 2297 & 418 & no \\
\hline PatJ & $\mathrm{A}_{4} \mathrm{~V}_{1} \mathrm{~B}_{2}$ & 7 & 7 & yes & 5 & 1.8 & yes & 314 & ndiWT & no \\
\hline Restin homolog ${ }^{2}$ & $\mathrm{Q}_{9} \mathrm{VJE}_{5}$ & 57 & 1.08 & yes & 70 & 1.05 & yes & 3 & 0.2 & no \\
\hline Herc2 & Q9VR91 & 3 & 0.8 & yes & 8 & 0.6 & yes & nd & & no \\
\hline Cindr & $\mathrm{Q}_{9} \mathrm{VA}_{3} 6$ & 1 & ndiWT & no & nd & & no & 23 & ndiWT & no \\
\hline Bottleneck $^{3}$ & $\mathrm{P}_{40794}$ & nd & & no & 1 & 0.5 & no & nd & & no \\
\hline Actin $\left({ }_{5} \mathrm{C}\right)$ & $\mathrm{X}_{2} \mathrm{JCP} 8$ & 301 & 1.1 & no & 181 & 0.8 & no & 247 & 0.7 & no \\
\hline Alpha Spectrin $^{2}$ & M9PGV6 & 181 & 1.02 & yes & 142 & 1.1 & yes & 3 & 0.05 & yes \\
\hline Alpha actinin & FoJAG6 & 70 & 1.1 & yes & 47 & 0.96 & yes & 12 & 0.3 & yes \\
\hline F-actin-capping protein subunit alpha & $\mathrm{Q}_{9} \mathrm{~W}_{2} \mathrm{No}$ & 19 & 1.2 & no & 24 & 1.2 & no & 12 & 0.4 & no \\
\hline F-actin-capping protein subunit beta & $\mathrm{P}_{4} 8603$ & 14 & 1.3 & no & 10 & 0.6 & no & 5 & 0.5 & no \\
\hline $\mathrm{Arpci}^{2}$ & $\mathrm{O}_{97182}$ & 9 & 1.1 & no & 9 & 0.7 & no & 3 & 0.5 & no \\
\hline $\operatorname{Arpc2}^{2}$ & $\mathrm{Q}_{9} \mathrm{VIM}_{5}$ & 20 & 1.1 & yes & 19 & 0.8 & yes & 3 & 0.1 & yes \\
\hline Arpc4 & $\mathrm{Q}_{9} \mathrm{VMH}_{2}$ & 4 & 1 & yes & 3 & 0.8 & yes & nd & & yes \\
\hline Arpc5 & Q9VQD8 & 5 & 1.1 & yes & 4 & 0.7 & yes & nd & & yes \\
\hline Arp2 & $P_{45888}$ & 8 & 1.5 & no & 7 & 0.8 & no & 5 & 0.6 & no \\
\hline Arp3 & $\mathrm{P}_{32392}$ & 18 & 0.97 & no & 17 & 1.06 & no & 9 & 0.5 & no \\
\hline Jaguar & $\mathrm{AoAoB}_{4} \mathrm{KGX}_{1}$ & 28 & 0.8 & yes & 44 & 1.04 & yes & 3 & 0.1 & yes \\
\hline Dilute class unconventional myosin & $\mathrm{A}_{1} 6 Z_{9}$ & 5 & 0.7 & yes & 7 & 0.5 & yes & nd & & yes \\
\hline Myosin-VIIa & $\mathrm{Q}_{9} \mathrm{~V}_{3} \mathrm{Z6}$ & 11 & 2 & yes & 13 & 0.9 & yes & nd & & yes \\
\hline Myosin II & Q99323 & 237 & 0.7 & yes & 266 & 1.2 & yes & 80 & 0.4 & yes \\
\hline
\end{tabular}

${ }^{1}$ not assigned to DROME but other Drosophila subspecies in sample A

${ }^{2}$ spectral counts for sample C (GFPSlam) have been set from o to 3 to avoid division by zero

${ }^{3}$ non-detectable in GFPSlam sample but measurable in WT sample below 3 spectral counts in sample A+C 


\subsubsection{Validity analysis of putative interactors}

\subsubsection{Arp2/3 complex}

The last 4 division of the nuclei before Drosophila cellularization during interphase of cycle 14 are occurring synchronously just beneath the plasma membrane at the cortex of the embryo. These last divisions (cycle 10-13) are characterized by a cyclic cytoskeletal rearrangement. During each interphase actin accumulates in caps above the nuclei. As the mitotic cycle proceeds these caps expand and fuse with neighbouring caps to form pseudocleavage or, indicating the timepoint, metaphase furrows. Thereby these transient furrows are separating centrosomes during mitosis. As cytokinesis is skipped, the furrows regress with mitotic exit and actin rearranges in the actin caps. The actin-nucleating factor Arp2/3 is a complex that is essential for the expansion of actin caps and thus the formation of metaphase furrows (Stevenson et al. 2002). As suggested by the crystal structure of bovine Arp2/3 complex, its functional assembly consists of Arp2, Arp3, Arpc1, Arpc2, Arpc3 (A and B, respectively in Drosophila), Arpc4 and Arpc5 (Robinson 2001). Arp2/3 nucleates branches at actin filaments at an angle of $70^{\circ}$. It is inactive until assembly through nucleation-promoting factors (NPFs like WASp and SCAR). During early interphase Arp3 localizes around the emerging actin caps in small perforated bands. As soon as the actin caps gather and begin to expand Arp3 localizes to the leading edge of the cap margins and, like actin, the emerging metaphase furrows. During cellularization Arp3 is localized apically and not at the FC. Since Arp3 is part of a functional complex the rest of the assembly likely colocalizes. For Arpc2 this is shown (Stevenson et al. 2002).

Like Arp3, Slam and its mRNA colocalize at metaphase furrows while mitosis and after mitotic exit, during interphase, in the intercap region (Schmidt, Lv, and Großhans 2018). In contrast to an unfunctional Arp2/3 complex (arpc1 mutant) however, there are no known abnormalities in slam mutants before cycle 14 (Stevenson et al. 2002; Thomas Lecuit, Samanta, and Wieschaus 2002; Acharya 2014). Localizing to the same sites one or more of the proteins of the Arp2/3 complex may have direct or indirect contact to Slam. Except Arpc3, all the proteins participating in the Arp2/3 complex have been enriched in the GFPSlam IP buffer sample 1. Arp3 has been enriched in all 3 GFPSlam IP samples, Arpcı in 2. Since the others have not been detected in most of the cases, they may have fallen under the detection limit. All this hints towards an attraction of the Arp2/3 complex and Slam may it be of direct or indirect kind. Also, the detergent containing RIPA(-) buffer GFPSlam sample A yielded slightly enriched Arpc1, 2, 5 and Arp2. Arpc2 appears to have direct contact to Anillin, MyoV (didum) and Actin (D'Avino et al. 2008; Guruharsha et al. 2011). Arpc1 was also shown to contact the mother actin filament (Guruharsha et al. 2011). Additionally F-actin capping protein subunit alpha and beta, both 
contact all the Arp2/3 complex proteins (Guruharsha et al. 2011). Apart from those interactions there are of course several contacts between the Arp2/3 complex subunits. It is possible that one of the abovementioned proteins has co-precipitated the Arp2/3 complex and that the interaction to Slam is of secondary, tertiary, or higher kind.

Unlike Slam, Arp3 does not localise to the furrow canal during cellularization. It stays apically, at the surface of the embryo (Stevenson et al. 2002). This makes a physical interaction unlikely during the cellularization process.

Regarding their functional involvements during mitotic cycle 10-14 of Drosophila early development it seems Arp2/3 complex passes the baton on to Slam: Arp2/3 acting in the establishment of a metaphase furrow and thus definition of the invagination site, and Slam, which is needed for furrow invagination during actual cellularization. Looking closer at possible interdependences would be interesting.

\subsubsection{MyoV (didum)}

Myosin V is an unconventional myosin. Conventional myosins (MyoII) are engaged in filament formation and force generation, whereas unconventional myosins are involved in cell motility, cytokinesis and trafficking (Yamashita 2000; Woolner and Bement 2009; Pocha, Shevchenko, and Knust 2011). Woolner et al. reported that unconventional myosins, particularly (mice MyoVa) MyoV, are acting as tethers for endosomes and membrane-associated proteins (like Slam) (Desnos et al. 2007; Provance et al. 1996; Wu et al. 1998; Woolner and Bement 2009).

Vertebrate and fly MyoV-motors exhibit well-preserved functions in cytoplasmatic transport but basic mechanisms appear to be different (Tóth et al. 2005).

Drosophila MyoV has been shown to facilitate the posterior localization of oskar mRNA in oocytes. MyoV is part of a transport competent RNP that, together with Kinesin, ensures right localization of oskar mRNA (Krauss et al. 2009). MyoV has been shown to interact with Kinesin heavy chain counterbalancing Kinesin function. This insures the beforementioned posterior localization of oskar mRNA and functional embryonic development of Drosophila (Krauss et al. 2009).

MyoV as well as Kinesin heavy and light chain have been detected in IP samples (MyoV: A (SC 142) and B (SC 12) only measured in GFPSlam sample, not detected in sample C; Kinesin light chain: detected in A $(\mathrm{FE} 2,5)$ but not in sample B or C, Kinesin heavy chain detected in sample B $(\mathrm{FE} 4,6)$ and in sample C (FE 1,1)). We know, that Slams localization to the FC depends on the centrosome controlled recycling endosome even though it does not directly localize with recycling endosome marker Rabı1 (Wilson et al. 2005; Acharya 2014). Slam and its mRNA are not 
only found at intercaps, metaphase and cellularization furrows but also in vesicle like basal particles near the FC (Schmidt, Lv, and Großhans 2018; Yan et al. 2017), which show a rather random than microtubule-dependent direct movement (Laupsien 2012). In Oskars case the RNA-binding protein Staufen promotes assembly and stabilisation of oskar RNP (Ramos et al. 2000; St Johnston, Beuchle, and Nüsslein-Volhard 1991; Ferrandon et al. 1994). It is not completely resolved if Slam itself binds its mRNA or if there is an associated RNA-binding protein, that contributes to the assembly of the complex that includes both. No known RNAbinding protein has been specifically coimmunoprecipitated with Slam in this study (except for a slight enrichment of IGF-II in RIPA- buffer sample A-C). Further we do not know how this RNP is transported to and entrapped at the furrow. Concerning the transport of oskar mRNA to the posterior pole of the embryo during stage 9 of development: Krauss et al. conclude that it is "long-range MT-based oskar mRNA transport throughout the cytoplasm, coupled to Kinesinindependent but MyoV-dependent short-range actin-based translocation to the cortex" (Krauss et al. 2009). Both MyoV and Kinesin have been found attached with Slam. It could be checked if Slam and slam mRNA localization would be affected by reduced MyoV activity (e.g. by a dominant negative MyoV protein like in Krauss et. al). Such an experimental approach could enlighten a possible relationship between Slam and its mRNA and MyoV, like it was observed for Oskar and its mRNA.

It has additionally been shown that MyoV specifically coimmunoprecipitates with Patj and the Crumbs complex during a study that reported how Crumbs regulated rhodopsin transport in the fly retina (Pocha, Shevchenko, and Knust 2011). Therefore, it is possible that MyoV was indirectly coimmunoprecipitated via a direct interaction with Patj. Several other interactors have been reported for MyoV. Among them Jaguar (Finan, Hartman, and Spudich 2011; Hurd et al. 2016), F-actin capping protein subunit alpha and beta (Guruharsha et al. 2011), Rabi1 (Ji et al. 2015) and, naturally, Actin (Guruharsha et al. 2011). It could be all of the abovementioned that coprecipitated MyoV. A possible physical interaction of Slam and MyoV could be investigated via an AP-crosslinking experiment to identify primary, secondary or tertiary interactors.

\subsubsection{Myosin II/Patj}

During mitotic cycle 10-13 of Drosophila development metaphase furrows transiently form to mature into furrow canals during cellularization. In both cases non-muscle Myosin II (zipper) is located at or near the furrow (Daniel P. Kiehart 1990; C. M. Field and Alberts 1995; Foe, Field, and Odell 200o). MyoII is an indispensable part of contractile actomyosin structures, which are one of the driving forces of cellularization. When the furrow starts to invaginate actin and 
myosin localize to the invagination front and form a network (Young, Pesacreta, and Kiehart 1991; Schejter and Wieschaus 1993; C. M. Field and Alberts 1995; Foe, Field, and Odell 2000; Royou et al. 2004). With continuing furrow ingression between cortical nuclei, the actomyosin network restructures into separate actomyosin rings. These rings then constrict to finish cellularization with basal closure (Schejter and Wieschaus 1993). MyoII and Slam localize to the leading edge of the invaginating furrow. Slam was reported to be involved and required for direct recruitment of MyoII to the furrow canal (Thomas Lecuit, Samanta, and Wieschaus 2002; Acharya 2014; B. He, Martin, and Wieschaus 2016). Also, Slam has been shown to ectopically localize MyoII when expressed after cellularization (Thomas Lecuit, Samanta, and Wieschaus 2002). MyoII was co-immunoprecipitated with Slam in 2 IP buffer (FE 10 and 2) samples and not detected in one. Taking co-localization and ectopic recruitment into account, this could point towards a direct interaction of MyoII and Slam. Patj, however, has been found to directly bind to spaghetti squash (Sqh), the regulatory light chain of MyoII, which also localizes to the furrow front (Sen, Nagy-Zsvér-Vadas, and Krahn 2012; Karess et al. 1991). Sqh is directly associated to MyoII (Vicente-Manzanares et al. 2009). It was proposed that Patj is affecting adherens junction stability by directly binding Sqh and subsequent recruitment of MyoII and further by inhibiting Myosin phosphatase and thus enhancing Myosin activity (Sen, Nagy-Zsvér-Vadas, and Krahn 2012). Patj has been shown to directly bind Slam (Wenzl et al. 2010). This was verified with this study. Patj was enriched in all 6 GFPSlam samples (IP and RIPA(-) buffer). This could mean, that the interaction of MyoII and Slam is of indirect kind. Either a direct binding assay or crosslinking experiments could shed light on the nature of this interaction.

\subsubsection{Restin homolog/Jaguar}

Restin homolog (also cytoplasmatic linker protein, CLIP19o) is the Drosophila homologue of human CLIP170 (Lantz and Miller 1998). It has been associated with the connection of vesicles and organelles to microtubule plus ends (Rickard and Kreis 1996). In pre- and cellularizing embryos it colocalizes with Lava Lamp (Lva) and $\alpha$-Spectrin to large cytoplasmatic puncta. Some of these puncta are located close to the furrow front. Restin homolog alone, however, is also observed at the furrow tips (Sisson et al. 200o). Lva is suggested (together with spectrin) to build a Golgi-based scaffold, which may facilitate an interaction of Golgi bodies with microtubule cytoskeleton and thus promote Golgi-derived membrane secretion, needed for the formation of new furrow during cellularization (Sisson et al. 2000).

Myosin VI, also Myosin $95 \mathrm{~F}$ or Jaguar is unique. Unlike all other known Myosins, it moves towards the pointed (minus) end of actin filaments (Cramer 200o). With its singularity, it is no 
surprise that Jaguar has been shown to be involved in a number of processes during the development of several organisms. It is required for stereocilia organization in human and mouse hair cells, which are maybe mostly known for their function in the inner ear (Tama Hasson et al. 1997; Self et al. 1999), for microvilli organization in Mouse and pig absorptive epithelial cells (T Hasson and Mooseker 1994; Heintzelman, Hasson, and Mooseker 1994; Self et al. 1999) and in Drosophila spermatogenesis in (Hicks et al. 1999), just to name a few. Earlier Jaguar has been found to ATP-dependent transport particles on actin filaments in the Drosophila embryo (Valerie Mermall, McNally, and Miller 1994). These particles are found at metaphase furrows. In embryos, where Jaguar function is experimentally impaired, profound defects of the syncytial blastoderm are observed. Actin caps and furrows are blurred and show abnormal spacing. Interestingly, similar to the Slam phenotype during cellularization, metaphase furrows do not invaginate deep enough to separate adjacent mitoses, as microtubule structures cross over them and spindles fuse. This leads to nuclear defects (V. Mermall and Miller 1995). The particles, that colocalize with Jaguar are found in cytoplasmic domains around the nuclei during interphase. When mitosis begins Jaguar is majorly found where metaphase furrows will form. As the transient metaphase furrows emerge, Jaguar gets less concentrated. This made Mermall and Miller conclude as early as 1995 , that " $95 \mathrm{~F}$ myosin (Jaguar) may be indirectly involved in furrow formation through the delivery of required components" (V. Mermall and Miller 1995). During Drosophila embryonic neuroblast development Jaguar is necessary for the localization of cell fate determinant and adaptor protein Miranda and their associated proteins. It has been suggested that Jaguar is involved in the translocation of the Miranda protein complex, which most interestingly also includes an mRNA (prospero mRNA/Staufen) (Petritsch et al. 2003).

For Slam and its mRNA it has been shown that the initial accumulation at the emerging furrow is vesicle dependent (Acharya et al. 2014). A colocalization with the recycling endosomes maker Rabil could not be observed at any timepoint. It is not known how Slam is transported to the membrane. The strong enrichment of Jaguar in two of the IP samples (50- and 29-fold) and the involvement in the transport of proteins of similar nature (Miranda, with Slam supposedly being an adaptor protein) may indicate a role of Jaguar in Slam translocation.

Both Restin homolog (CLIP-190) and Jaguar have been co-immunoprecipitated with Slam (Restin homolog 3/3 IP samples and Jaguar 2/3 IP samples). Further, Jaguar and Restin homolog have been found to coexpress in several tissues during embryogenesis in Drosophila (Lantz and Miller 1998). In neurons they colocalize in vesicle like particles, actin cytoskeleton-dependent at the posterior pole during early Drosophila development and to organelles in several cell types (Lantz and Miller 1998; Beaven et al. 2015). They co-immunoprecipitated and colocalize in the 
embryonic nervous system, both actin- and microtubule-dependent (Beaven et al. 2015). The function of Restin homolog and Jaguar interaction is not known (Lantz and Miller 1998; Finan, Hartman, and Spudich 2011; Beaven et al. 2015). It was suggested that this connection of a myosin and a microtubule-associated protein may link the actin und microtubule cytoskeleton. Slam, Restin homolog and Jaguar are all localized at or near the emerging furrow during cellularization and somewhat support the growth of the emerging furrow. Restin homolog is mainly known for its role in linking vesicles to microtubules. The function of the interaction of Jaguar and Restin homolog is unclear. The connection between all 3 can be either an intermediate binder (e.g. MyoV, both MyoV and Jaguar were reported to co-precipitate with Long Oskar (Hurd et al. 2016)) or the dependence of Slams accumulation at the furrow on vesicle transport is somehow associated to Jaguar and Restin homolog. This may be addressed in further studies.

\subsubsection{CD2AP/CIN85 related (Cindr)}

The CD2AP/CIN85 family of adaptor proteins is suggested to mainly function in endocytosis to down-regulate receptor tyrosine kinase activity (Dikic 2003); but also, have been described to interact with other trafficking proteins like Rab4, PAK2 and Dab2 (Cormont et al. 2003; Kurakin, $\mathrm{Wu}$, and Bredesen 2003; Kowanetz, Terzic, and Dikic 2003). Furthermore, a relationship between $\mathrm{CD}_{2} \mathrm{AP} / \mathrm{CIN} 85$ proteins and actin has been proposed, as they were detected in actinrich regions of podocytes and cultured cells. They have been found to bind actin in vitro and support actin bundling (Welsch et al. 2001; Lehtonen, Zhao, and Lehtonen 2002; Gaidos et al. 2007). CD2AP was shown to bind F-actin capping protein CAPZ in T-cells and inhibit its function in vitro (Hutchings et al. 2003; Bruck et al. 2006) . Furthermore it is reported to bind anillin at the cleavage furrow (Monzo et al. 2005). Most interestingly the CD2AP/CIN85 family has been linked to E-cadherin. It has been shown, that CD2AP interacts with E-Cadherin in vitro via its $\mathrm{SH}_{3}$ domain (Lehtonen et al. 2004; Mustonen et al. 2005). In Drosophila it was reported that Cindr is needed for normal localization of E-Cadherin and Roughest in the retina. In the same study Cindr has been co-immunoprecipitated with F-actin capping protein alpha and beta. Here, a role of Cindr in intermediating between junctions and actin cytoskeleton was proposed. (Johnson, Seppa, and Cagan 2008).

During cellularization, YFP-tagged Cindr has been shown to localize to the front of the invaginating membrane (basal junctions and furrow canal could not be distinguished at this point) to become cytoplasmatic after the cells have been encapsuled (Lye, Naylor, and Sanson 2014). In Drosophila S2 cells Cindr localizes to cleavage furrows, intercellular bridges and midbodies. It colocalizes with Anillin during the whole cytokinesis and throughout Drosophila 
development (Haglund et al. 2010). A reduction of Cindr (RNAi) in S2 cells results in 3-fold binucleate cells, assigning a certain function during cytokinesis. This was similarly reported for human cells (Haglund et al. 2010). The $\mathrm{SH}_{3}$ domains of mammalian $\mathrm{CD}_{2} \mathrm{AP} / \mathrm{CIN} 85$ family members interact with $\mathrm{Px}(\mathrm{P} / \mathrm{A}) \mathrm{xxR}$ motifs (Kowanetz et al. 2003; Kurakin, $\mathrm{Wu}$, and Bredesen 2003). Such a motif was found within the Anillin sequence and proposed as binding site. During co-IP experiments, when Anillin was divided into 4 GST-tagged parts, Cindr indeed associated with Anillin's Px(P/A)xxR motif (PLARLR, amino acids 145-150) containing part (Haglund et al. 2010). The interaction was impaired when the consensus arginine was mutated to alanine (R15oA). Since Cindr was co-precipitated with Slam in IP buffer samples and even under harsher conditions with RIPA(-) buffer (5 of 6 and not detected in 1) and Cindr was found to localize to the cellularization front, the Slam sequence was checked for a $\operatorname{Px}(\mathrm{P} / \mathrm{A}) \mathrm{xxR}$ motif. Indeed 2 of these motifs were found within the Slam (LD36405p) sequence. The first one was between amino acids 392 and 397 (PQPRPR) and the second between amino acids 565 and 570 (PVPAPR). Taken together this strongly indicates a direct interaction of Slam and Cindr via its $\mathrm{SH}_{3}$ domain. Testing this hypothesis could be approached as described for Anillin in Haglund et al. 2010. The motif containing parts could be cloned and tagged. Subsequently one can check if the respective Slam fragments can coimmunoprecipitate Cindr (from embryonic lysate or S2 cells for instance) compared to non-motif parts of Slam. Further it could be tested if a mutation within the consensus sequence would impair these potential interactions.

During mitotic cycle 10-13 cytoplasmatic buds rich in villous projections are repeatedly separated by flat invaginations of the plasma membrane, the metaphase furrow. The metaphase, or pseudocleavage furrow, contains specific junctional proteins among them E-cadherin/ $\beta$-catenin complexes, MyoII, Patj and Slam, as well as Dlg and Scribbles (Hunter and Wieschaus 2000; Thomas Lecuit, Samanta, and Wieschaus 2002). As invagination proceeds during actual cellularization these proteins separate into proteins marking the cellularization front (Slam, Patj, MyoII) and junctional proteins localizing at (E-cadherin/ $\beta$-catenin) and apically to basal adherens junctions (BAJ; Dlg/Scribbles)(Thomas Lecuit 2004). It was shown that Cindr, as well as Slam, localizes to the cellularization front and that Cindr coimmunoprecipitates with E-cadherin. E-cadherin shares the localization to the metaphase furrows with Cindr and Slam. To clarify if Cindr moves forward with the invaginating membrane during cellularization or stays somehow associated to or near to the BAJ would be advised. Since the formation of the BAJ is dependent on Slam, it would also be absorbing to know if Slam might be somehow mediating between Cindr and junctional proteins during metaphase furrow formation (Lecuit et al., 2002). 


\subsubsection{Bottleneck}

The gene bottleneck (bnk) has been characterised during zygotic screens. Bottleneck has been shown to determine the timepoint of basal closure by regulating the actomyosin network (Schejter and Wieschaus 1993). Mutations of bnk lead to premature contraction of the microfilament network before the cellularization front has passed the basal end of the nuclei. Thus some nuclei are trapped within the constricted actomyosin rings and assume an hourglasslike shape (Schejter and Wieschaus 1993; Theurkauf 1994). In some cases, the nuclei are pushed away from the emerging cell and short cells without nuclei form. Similar to zygotic slam, first bnk transcripts are detected during mitotic cycle 11 (Schejter and Wieschaus 1993). Subsequently expression undergoes rapid upregulation and mRNA levels reach a peak just before cellularization to quickly decrease in the following (Thomas Lecuit 2004; Reversi et al. 2014). Bottleneck colocalizes to the furrow front with F-actin and Myosin II during slow phase of cellularization (Schejter and Wieschaus 1993; Thomas and Wieschaus 2004). Bottleneck is a 300 amino acid protein, which is extremely basic and, just like Slam, with no known protein domains (Reversi et al. 2014). There are no interactors reported for Bottleneck (flybase.org).

Reversi et al. have been exploring a possible mechanism of how Bottleneck determines the timing of basal closure (Reversi et al. 2014). They found that Bottleneck together with $\mathrm{PI}(3,4,5) \mathrm{P}_{3}$ appears to counteract $\mathrm{PI}(4,5) \mathrm{P}_{2}$ dependent-contractility of the actomyosin network during cellularization. In this regard, Bottleneck proposedly cross-links actin and limits myosin recruitment to the plasma membrane until it is time for actomyosin ring contraction and thus, basal closure.

Bottleneck was found enriched 33-fold in one of the IP samples (A) and stayed undetected in the other 2 samples (B and C). Since Bottleneck likely fell under the detection limit in sample B and $C$ it has to be considered as a Slam interactor of either direct or indirect kind. Apart from their similar expression pattern during the onset of and their major role during cellularization, Bottleneck and Slam have more things in common. They both localize to the furrow front during slow phase of cellularization and most importantly they seem to have an interface in seriously influencing myosin levels at the furrow front. In this regard, they are reported to have counteracting roles: Bottleneck restricts Myosin II levels at the furrow front and Slam recruits Myosin II to the furrow canal. In one point however, they collaborate: to ensure plasma membrane invagination beyond the basal end of the nuclei in order guarantee single unit epithelial cells. 


\subsubsection{Herc2/Neurl4}

The probable ubiquitin-protein ligase Herc2 and its homologue and substrate Neuralized E3 ubiquitin protein ligase 4 were both enriched in all 3 IP samples (Herc2: FE 46, 16 and 5 for sample A, B and C respectively; Neurl4: FE 11 in sample A, SC 6 and 2 and not detected in wildtype for sample B and C). Herc2 and Neurl 4 were shown to associate with $\mathrm{CP} 110$, a centrosome component involved in centrosome biogenesis (Al-Hakim et al. 2012). The complex of ubiquitin ligase Herc2, the adaptor protein $\mathrm{Neurl}_{4}$ and $\mathrm{CP} 110$ were reported to regulate centrosome architecture in human cells. Neurl4 lacks an E3 ligase domain and is characterised by 6 Neuralized Homology Repeats (NHR domain), which is thought to be mediating protein-protein interaction (J. Li et al. 2012; F. He et al. 2009). Neurl4, which therefore likely lacks ligase activity was shown, however, to promote CP110 ubiquitylation in human cell lines (J. Li et al. 2012).

In Drosophila Neurl 4 was first identified during blastoderm development where it was shown to disrupt the localization of oskar mRNA (J. Li et al. 2012). Neurl4 in Drosophila also localizes to centrosomes and downregulates CP110, which suggests a similar role in flies (J. Jones and Macdonald 2015). Also a specific role in germ-cell morphology was reported (J. Jones and Macdonald 2015). By the beforementioned Neurl4/CP11o biochemical pathway it seems to affect PGC morphology and number. In this case it is not clear if Neurl4 is subject to Herc2 ubiquitination and subsequent downregulation as in mammalian cells. An interaction of Herc2 and Neurl 4 has not been reported for Drosophila. The coimmunoprecipitation of Herc2 and Neurl 4 with Slam is an indication for a similar relationship of Herc2/Neurl 4 and $\mathrm{CP}_{110}$ as reported for mammalian cells.

Although there has no specific function been assigned to both proteins during cellularization, an interaction of Herc2/Neurl 4 and Slam has to be considered with respect to the outcome of the mass spectrometric analysis of GFPSlam co-IP samples. There is, however one connection between Herc2/Neurl4 and Slam: Notch signalling, a pathway for intercellular communication mainly during cell fate decision (Bray 2006) Herc2 and Neurl 4 have both been shown to be involved in Notch signalling, through the endosomal pathway (Imai et al. 2015).

Notch signalling is initiated in the course of cellularization (Falo-Sanjuan et al. 2019). Specification and ingression of the furrow during cellularization requires Slam and Nullo, which both localize to the basal part of the FC (Hunter and Wieschaus 20oo; Thomas Lecuit, Samanta, and Wieschaus 2002). Notch and Delta were reported to be present on the growing lateral membrane (Falo-Sanjuan and Bray 2021). In the same study (preprint) it was shown that the notch signalling onset is membrane growth, and thus, Slam-dependent. If there is any rather direct correlation between the notch signalling onset through Herc2 and/or Neurl4 and Slam, 
must be subject of further studies. Another possibility is, that Slam is ubiquitinylated by Herc2 either for regulatory purposes or degradation. This may be mediated by adaptor protein Neurl 4 . It is also possible that Herc2 or Neurl4 pulled down the other since they are known interactors. Both proteins have not been investigated much in Drosophila. There sure is still a lot to explore for ubiquitin ligase Herc2 and its substrate Neurl4.

\subsection{Post-translational modifications and stability of Slam}

\subsubsection{Post-translational modification}

If Slam is isolated from its original organism, D. melanogaster, it runs higher than its expected molecular weight during SDS-PAGE ( $180 \mathrm{kDa}$, calculated MW $133 \mathrm{kDa})$. Also, it runs in a double band (figure 28). One reason could be a post-translational modification. Precisely, the double band could be the result of multiple phosphorylation. To test Slam for phosphorylation an assay was conducted: staged fly embryos were collected and lysed. One lysate was phosphatasetreated, the control stayed untreated. The result is shown in figure 29 and 30.

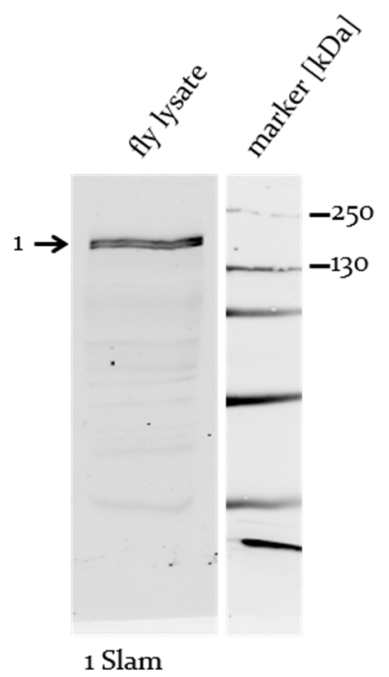

Figure 28 Slam double band. Western blot and immunostain for Slam of staged fly embryos (1.5-2.5h). Flies were harvested during cellularisation stage and lysed. The proteins in the lysate were separated via SDS-PAGE and blotted on a nitrocellulose membrane. The blotted proteins were then stained for Slam.

On the western blot in figure 293 samples are depicted. The input sample, the phosphatase treated (+ LambdaPP) and non-treated (- LambdaPP) control. Slam usually appears in a double band, which is slightly visible in the input sample. The lower band is very close to and fainter than the upper band. In contrast to this, another band emerges upon phosphatase treatment, which corresponds to a smaller molecular weight than the usually appearing lower band of the Slam double band (see figure 29). The non-treated sample only shows a shadow at the corresponding molecular weight. Even though the western blot has only poor quality this hints towards a possible post-translational phosphorylation of Slam. 


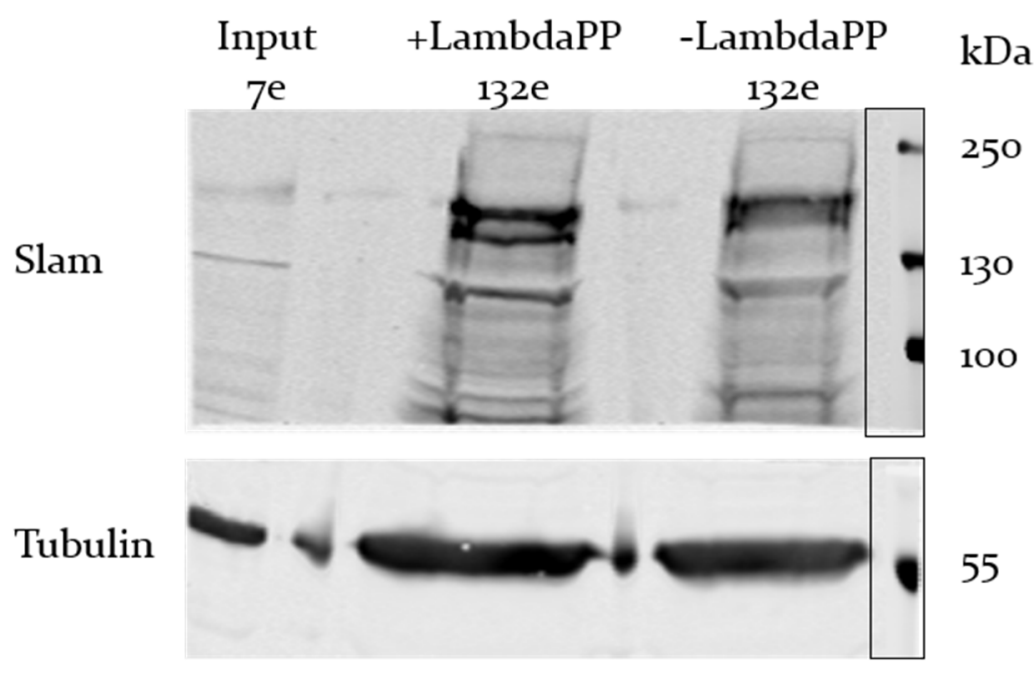

Figure 29 Putative phosphorylation of Slam. Western blot to detect Slam and $\alpha$-tubulin (as loading control) in samples of the phosphorylation assay. Samples were separated by SDS-PAGE (8\%). For lane 1-3 (marked Input, + LambdaPP and - LambdaPP) the loaded embryo equivalents (e) are listed. To lane 4 the protein weight reference marker was applied. The phosphatase treated sample shows an additional lower weight band, which is not visible in the control or the input sample.

Owing to the bad quality of the western blot the analytical procedure was repeated. The percentage of the SDS-PAGE gel was changed to $6 \%$ and a comb with bigger wells was used to achieve a better horizontal separation of the bands. A shortage of input sample explains the repeatedly low amount of loaded embryo equivalents. The result is shown in figure 30.

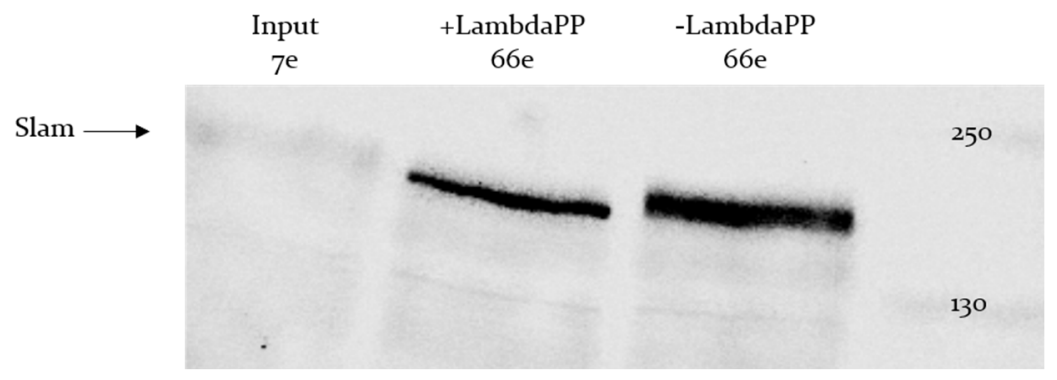

Figure 30 Analytics repetition of phosphorylation assay samples. Analytics (SDS-PAGE (6\%), western blot, immunostaining) were repeated for samples in figure 29 to detect Slam and $\alpha$-Tubulin in LambdaPP-treated and non-treated samples. Lane 4 contains the reference proteins.

The band of the input sample is faint but detectable. The two Slam bands did not separate as they did during the SDS-PAGE in figure 29. Even though the bands did not separate it is visible that the Slam band in the phosphatase-treated sample is thinner, also compared to the vague input band. The band thinned down to the lower part which indicates a loss of mass and/or change in migration behaviour during SDS-PAGE. Since the sample was treated with a 
phosphatase, Slam may very well be phosphorylated. However, to state that with certainty other assays must be conducted. Mass spectrometry or phospho-specific antibodies could be utilized for further experiments.

Protein phosphorylation is one of the most common post-translational modification (PTM)(X. $\mathrm{Li}$ et al. 2013, 20; Sacco et al. 2012). The reversible process of phosphorylation and dephosphorylation is involved in countless regulatory processes (H. S. Lu et al. 2012; Ardito et al. 2017). Protein kinases phosphorylate and protein phosphatases dephosphorylate. By this means the target protein is activated/deactivated and thus undergoes an upstream regulation and may further control downstream effectors. Since the preceding results indicated a phosphorylation of Slam and mass spectrometric results also displayed multiple phosphorylations (data not shown, mass spectrometry analyses in cooperation with Christof Lenz and Bernhardt Schmidt) the double band of Slam was investigated during the first hours of Drosophila development.

Wild-type embryos have been collected in an hourly timeframe till after cellularization. The embryos were flash-frozen in liquid nitrogen immediately and stored at $-80^{\circ} \mathrm{C}$ until analysis. $1 \mathrm{x}$ loading dye (Laemmli) was directly added to the samples. The embryos were lysed in $1.5 \mathrm{~mL}$ sample tubes using a small homogenizer. Samples were heated to $95^{\circ} \mathrm{C}$ for $5 \mathrm{~min}$, spun down and applied to a 10\% SDS-PAGE gel. After SDS-PAGE the proteins were blotted to a nitrocellulose membrane and immunostained for Slam and Tubulin (as a control).

The Slam double band appears to change during the first hours of embryogenesis. slam has its peak of expression at the onset of cellularization and the protein is stable throughout the process and rapidly degraded afterwards. The quantity of Slam during cellularization reflects the expression profile (fig. 31). Cellularization of the blastoderm starts approximately $130 \mathrm{~min}$ after fertilization of the egg and lasts about an hour (Campos-Ortega and Hartenstein 2014). This is exactly where most of Slam is detected. In the next hour (3-4 h) the majority of protein is degraded. The Slam double band appears to have a similar strength at $\mathbf{1 - 2} \mathbf{h}$, before cellularization. Then, during cellularization, the lower band proposedly dephosphorylated band, seems clearly stronger than the upper and putatively phosphorylated band. This could be due to degradation, but the two bands again show similar strength after cellularization (3-4 h). At 4$5 \mathrm{~h}$ the bands that are detected by an antibody against the N-terminal third of Slam appear in a different pattern. The upper band is undetectable, and the lower band seems to have made a slight shift upwards. The increased strength of the lower band could be due to regulative dephosphorylation. It is possible that the dephosphorylated or less-modified version of Slam is in an active state and can fulfil its task at the furrow front. Together serine and threonine represent almost $1 / 5$ of Slams amino acids (serine $12,2 \%$ and threonine $6.4 \%$, 
ProtParam)(Gasteiger et al. 2005). There is room for manifold phosphorylation. The band at Slams calculated weight at about $130 \mathrm{kDa}$ doesn't undergo striking changes apart from the usual upregulation of slam at the onset of cellularization. At this point it is not assumed that there is a direct interaction between Slam and its RNA, however, it cannot be completely ruled out. It is well known that a lot of RNA-binding proteins involved in pre-mRNA splicing and mRNA decay undergo a regulation by phosphorylation (Thapar 2015). The phosphorylation effects the ribonucleoprotein assembly. During an immunostaining on a Western blot against the Nterminal third of Slam it mainly appears in the two upper bands which are separated by an estimated weight of a few kDa. The upper band seems quite stable in strength but is also influenced by the slam upregulation during cellularization and the degradation after. In contrast the lower band appears much stronger during the course of cellularization, which means this state of Slam seems to be needed during the process. May be, that these two states of Slam serve different functions. The upper band Slam has a function, that is also needed prior to cellularization and the lower band Slam one that is also required during syncytial cycles but mainly during cellularization. For the upper band Slam there may be a role in recruitment and spacial specification of mRNA and the lower band Slam may rather be involved as adaptor protein and in the recruitment of Patj, RhoGEF2, MyoII and other proteins needed at or near the furrow front during cellularization. This is purely hypothetical and needs to be untangled during further research on Slam and its biochemistry during early Drosophila development. There is proof however that Slam is at least partially phosphorylated and the phosphorylation assay and the quantitative profile of Slam during cellularization hint towards a stronger phosphorylation and even regulation through phosphorylation and dephosphorylation before and during cellularization. Furthermore, Slam has high phosphorylation potential due to his richness in serine and threonine.

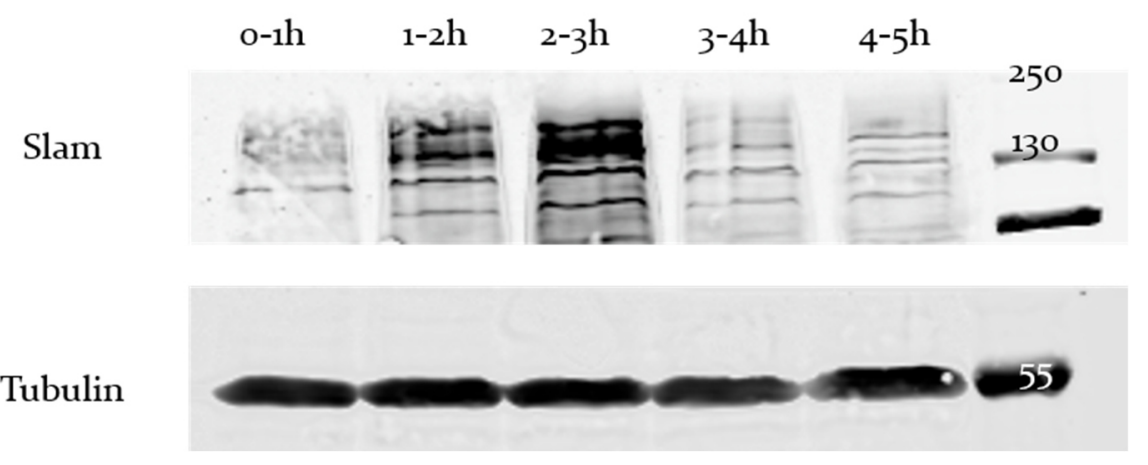

Figure 31 Quantitative profile of Slam during the first hours of Drosophila deleopment. 80 e have been applied for each sample. The upper and lower band seem to change in ratio during the early fly development. Where the bands show similar strength before and after, the lower band seems much stronger during the timecourse of cellularization ( 130-180 min after fertilization, 2-3 h). After basal closure Slam is rapidly degraded (3-4 h). 


\subsubsection{Stability of Slam}

\subsubsection{Stability in lysate}

In a lysate of cellularizing fly embryos Slam degrades gradually but full-length Slam will be detectable for a considerable amount of time. In figure 32 a western blot is depicted, which is stained for Slam. Staged fly embryos were lysed in RIPA(-) buffer (including AEBSF and complete protease inhibitor). An input sample was taken, and enzymatic reactions immediately stopped by the addition of Laemmli buffer and an incubation at $99^{\circ} \mathrm{C}$ for $5 \mathrm{~min}$. The lysate was incubated at $30^{\circ} \mathrm{C}$ and samples were taken at $1 \mathrm{~min}, 2 \mathrm{~min}, 3 \mathrm{~min}, 4 \mathrm{~min}, 5 \mathrm{~min}$ and $10 \mathrm{~min}$. Sample proteins were separated by SDS-PAGE and a Western blot was conducted to visualize Slam protein.

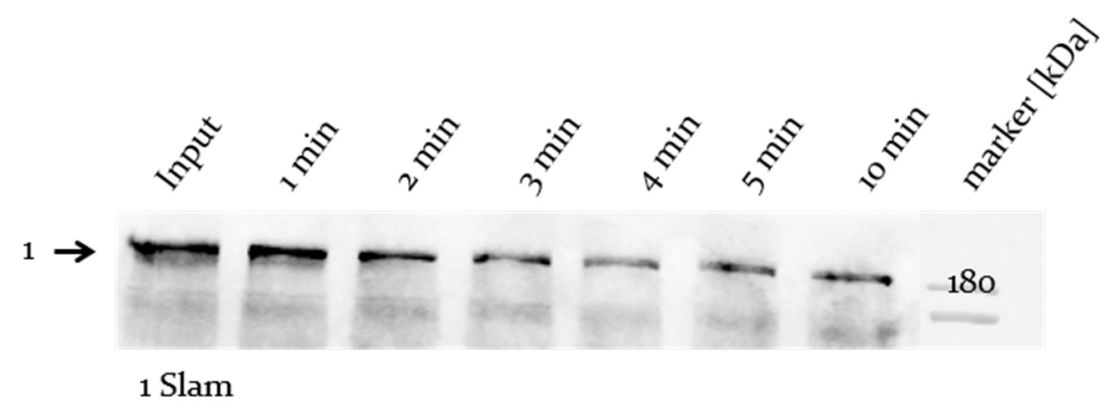

Figure 32 Slam degrades gradually in an embryonic lysate. Stability of Slam in a lysate of cellularizing Drosophila embryos: The lysate was incubated at $30^{\circ} \mathrm{C}$ for the respective amount of time. The input sample was taken directly after lysis. 80 embryo equivalents were applied to each lane. Proteins were gel electrophoretically separated and blotted to a nitrocellulose membrane using semi-dry transfer. The blot was stained with an anti-Slam (N-terminally) antibody (guinea pig) and visualized with IRDye secondary antibody (anti-Guinea pig $800 \mathrm{CW}$, Biorad). The quantity of full-length Slam slowly decreases. The lower band of the Slam double band degrades first. The upper band seems more stable under beforementioned conditions.

Electrophoretically separated in a polyacrylamide gel, Slam runs at a higher molecular weight than expected. The calculated weight of Slam is $133 \mathrm{kDa}$. The observed molecular weight during an SDS-PAGE is approximately $180 \mathrm{kDa}$. The difference is almost $50 \mathrm{kDa}$. To investigate if Slam would also exhibit a higher molecular weight during a gel filtration, $190 \mathrm{mg}$ cellularizing Drosophila embryos were lysed in $1 \mathrm{~mL}$ RIPA buffer (including complete protease inhibitor). After clarification of the crude lysate, $250 \mu \mathrm{L}$ sample were applied to a Superdex 200 (HR 10/30) column in RIPA buffer. $1 \mathrm{~mL}$ fractions were collected. The protein of $400 \mu \mathrm{L}$ of each fraction were acetone precipitated and analysed (SDS-PAGE, Western blot, immunostain for SHMT and Slam). The result is shown in figure 33. The separation range of Superdex 200 is $10^{4}-6 \times 10^{5} \mathrm{kDa}$. Slam elutes right after the void volume, which indicates a molecular weight (MW) of around $600 \mathrm{kDa}$. 
A

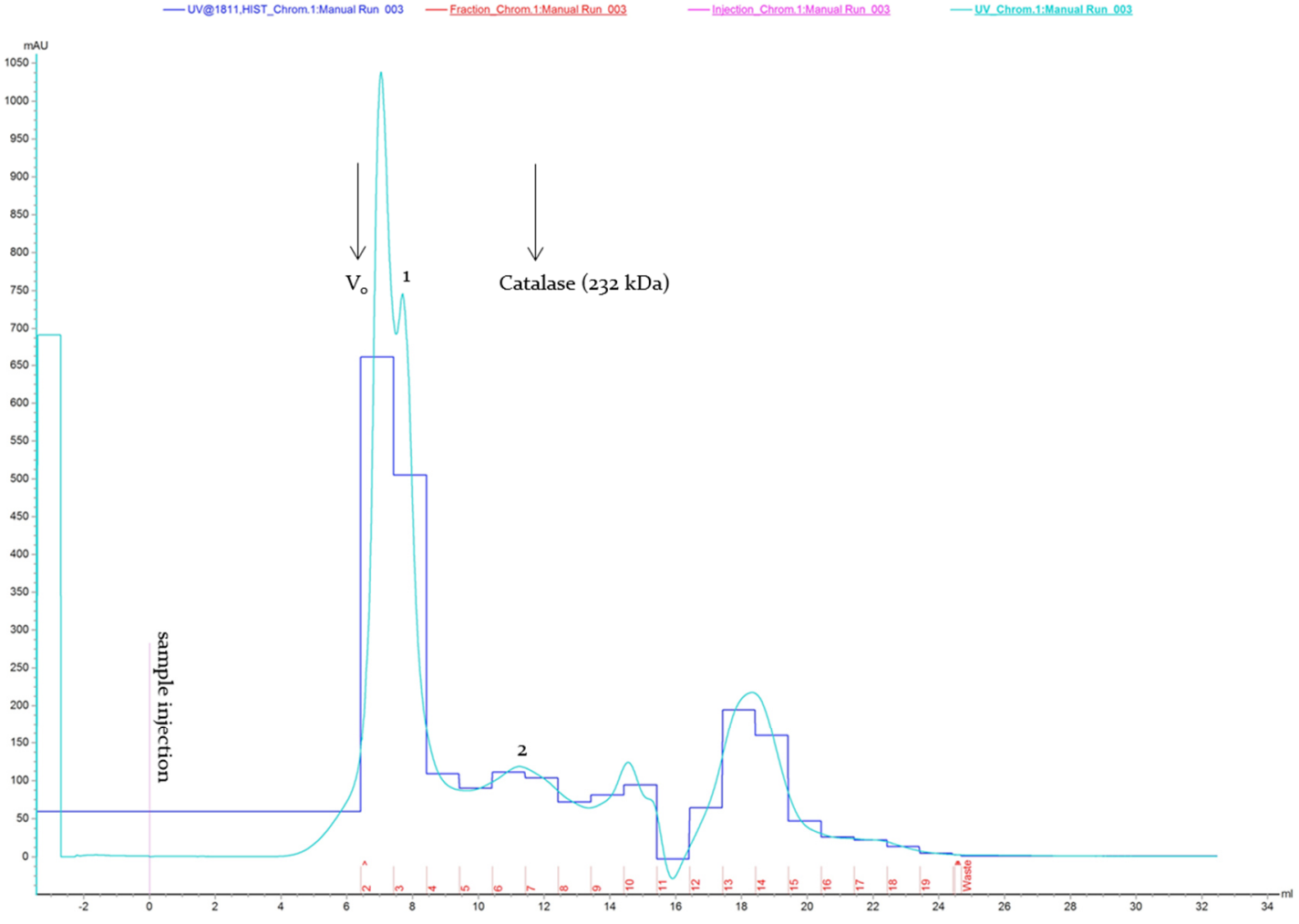

B

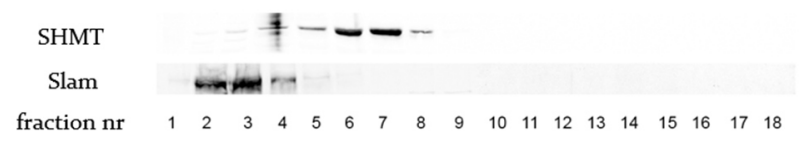

Figure 33 Chromatogram (A) and analysis (B) of a Superdex 200 gel-filtration of Drosophila (WT) embryonic extract (1.5$2.5 \mathrm{~h}$ ). $250 \mathrm{\mu L}$ sample were injected. $1 \mathrm{~mL}$ fractions were collected (fraction numbers are indicated), and $0,4 \mathrm{~mL}$ of each fraction were acetone precipitated and redissolved in Laemmli buffer. The analytical process included an SDS-PAGE to separate sample protein followed by a Western blot and an immunostain for Slam and SHMT. Slam elutes shortly after the void volume $\left(V_{0}\right)$ under peak 1 (A) and must therefore be part of an assembly that has a size close to $600 \mathrm{kDa}$ (separation range Superdex 200: $10^{4}-6 \times 10^{5} \mathrm{kDa}$ ). SHMT elutes with peak 2. The elution volume of SHMT corresponds to a MW (globular protein) of approximately 250 kDa (Winkler et al. 2017).

Slam colocalizes with its mRNA, also during ectopic localization. Slam was found to be necessary for its mRNA to localize to its site of action: the basal region of the furrow canal. There is a codependency between Slam protein and its mRNA. Slam is necessary for the mRNA to localize to the emerging furrow canal. The mRNA, however, is required to obtain a sufficient amount of Slam protein to fulfil its, yet unknown, task, which eventually leads to successful cellularization of the embryo (Yan et al. 2017). Slam and slam mRNA specifically co-precipitate during immunoprecipitation experiments. This indicates that Slam and its mRNA are part of a complex. Since Slam does not display any RNA binding motif, it may be an RNA-binding protein involved (Yan et al. 2017). To test if the RNA effected the elution volume of Slam during a gel filtration of 
an embryonic lysate, it was treated with RNase before column application. For that, $100 \mathrm{mg}$ wild-type embryos were lysed. An input sample was taken (10 $\mu \mathrm{L}$ sample plus $10 \mu \mathrm{L}$ Laemmli buffer). $1 \mu \mathrm{L}$ of RNase (10 mg/mL) was added to the lysate and incubated $10 \mathrm{~min}$ at RT. A sample was taken. A third sample was taken just before column application. It was proceeded with the gel filtration. $250 \mu \mathrm{L}$ sample were applied to the column. Flow rate was adjusted to $500 \mu \mathrm{L} / \mathrm{min}$. $1 \mathrm{~mL}$ fractions were collected. The fraction samples were treated and analysed as described in the beforementioned gel filtration procedure. The result (figure 34) was unexpected. RNase treatment of the lysate had a strong effect on Slam: it was undetectable by Western blot after a 10 min treatment at RT. In figure 32 the stability of Slam in lysate is demonstrated. It was clearly detectable on a Western blot after a 10 min incubation at $30^{\circ} \mathrm{C}$. The undetectability of Slam can also not be explained by the dilutional effect of a gel filtration. The samples were acetone precipitated and the protein therefore reconcentrated. Additionally, Slam was visible in the preceding gel filtration and SHMT had a similar signal strength.

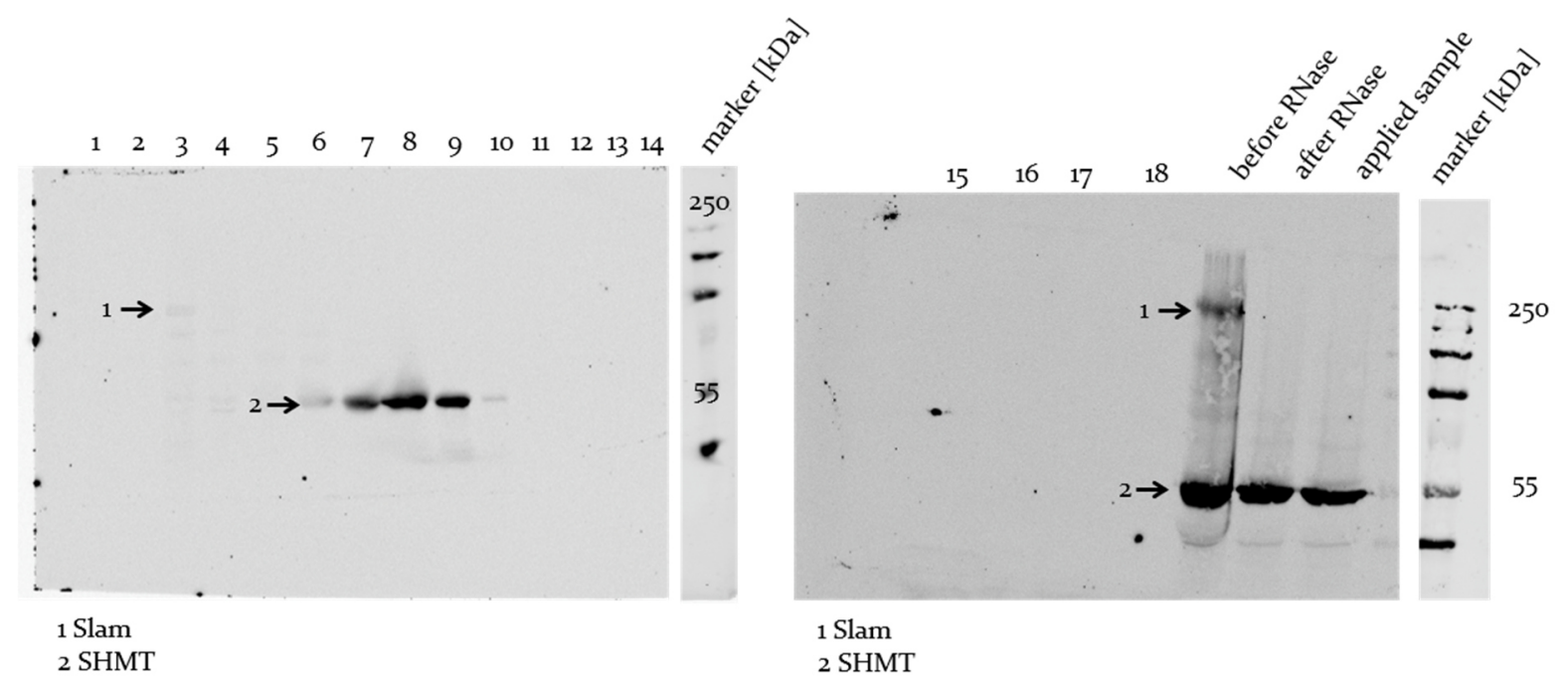

Figure 34 Slam is almost undetectable in samples after RNase treatment. Western blot (stained for Slam and SHMT) of samples from gel filtration of Drosophila embryonic lysate after RNase treatment. Fraction numbers are indicated. Before the RNase treatment Slam was visible and seems completely vanished after a 10 min treatment with DNase-and protease-free RNase. A shadow of signal was detectable of Slam in fraction 3 (indicated by an arrow labelled with 1). SHMT is unaffected of the treatment and elutes with the same volume of preceding gel filtrations.

\subsubsection{Slam's stability decreases in the presence of RNase}

To probe if Slam's stability was dependent on the presence of additional RNase, first the amount of RNase was titrated to the lysate. For this, $91 \mathrm{mg}$ of cellularizing fly embryos were lysed in $1 \mathrm{~mL}$ RIPA(-) buffer. RIPA(-) has no SDS and lower salt than conventional RIPA. RIPA(-) will not 
disturb the (possibly indirect) contact between Slam and RNAs (Dr. Shuling Yan). An input sample was taken (100 $\mu \mathrm{L}$ lysate plus $17 \mu \mathrm{L} 6 \mathrm{x}$ Laemmli). The further setup was as follows (table 8).

Table 8 Titration of RNase in fly embryo lysate

\begin{tabular}{|l|l|}
\hline sample & treatment \\
\hline input & $100 \mu \mathrm{L}$ lysate $(2 \mathrm{e} / \mu \mathrm{L})$ \\
\hline control & $100 \mu \mathrm{L}$ lysate $+1 \mu \mathrm{L}$ ddH $\mathrm{O}_{2}(2 \mathrm{e} / \mu \mathrm{L})$ \\
\hline RNase & $100 \mu \mathrm{L}$ lysate + respective amount of RNase \\
\hline
\end{tabular}

After an incubation of 10 minutes at RT, the reaction was stopped by the addition of Laemmli buffer and $5 \mathrm{~min}$ at $95^{\circ} \mathrm{C}$. Sample proteins were electrophoretically separated and blotted on to a nitrocellulose membrane (semi-dry transfer). The blot was stained for Slam and SHMT, respectively. In figure 35 the result of sample analysis is depicted. RNase incubation had little or no effect on SHMT. The band, which was incubated with the highest amount of RNase (1o $\mu \mathrm{g})$ appears sharper than the rest of the bands. Since the aforementioned band also shows a stronger stain, it cannot be concluded, that there was less SHMT present. Slam, however, is strongly affected by the presence of RNase. A 10 min incubation (RT) with $3 \mu \mathrm{g}$ or higher, will make Slam disappear in a western blot. This means the amount of protein has fallen below the detection limit of immunostaining on a western blot, which is around $100 \mathrm{pg}$. The control shows that molecular movement or proteolytically active enzymes in the lysate will lead to the degradation of a certain amount of protein. The addition of RNase increases this degradation distinctly. The amount of RNase, that is needed to see an effect on Slam has been titrated to an amount of o,3 $\mu \mathrm{g}$ in $100 \mu \mathrm{L}$ lysate. 


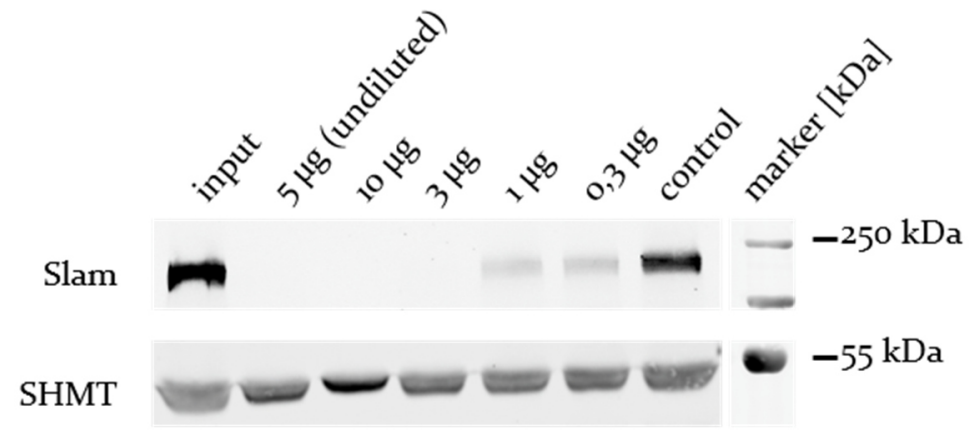

Figure 35 Titration of RNase to the lysate of cellularizing fly embryos. Respective amounts of protease-and DNase-free RNase (Thermo Fisher Scientific) was added to a fresh embryonic lysate and incubated for 10 min at RT. The reaction was stopped by addition of Laemmli buffer and an incubation of the mixture at $99^{\circ} \mathrm{C}$ for $5 \mathrm{~min}$. Proteins in the samples were separated by SDS-PAGE (8\%), transferred to a nitrocellulose membrane but semi-dry western blot and immunostained against Slam and SHMT as control. The input sample was taken directly after lysis and the control sample was incubated without RNase (see table 8). Overall, the amount of SHMT was not affected by the RNase. The more RNase was added, the less Slam was detected after the incubation time. The control sample showed less Slam than the input sample, but clearly more than the RNase-treated samples. The approximate amount of RNase for $100 \mu \mathrm{L}$ sample was titrated to $0,3 \mu \mathrm{g}$.

Is it really the RNase, that makes Slam more prone to degradation? To ascertain the effect of the RNase on Slams stability the experiment was repeated with an additional sample that contained RNase inhibitor (table 9). The degradation of Slam in the presence of RNase appears to be very fast. Hence, the RNase and its inhibitor were preincubated ( 5 min on ice). In this experiment $50 \mu \mathrm{L}$ sample were treated with $0.1 \mu \mathrm{g}$ of RNase.

Table 9 Samples for the verification of the influence of the presence of RNase on Slam stability.

\begin{tabular}{|l|l|}
\hline sample & treatment \\
\hline input & $50 \mu \mathrm{L}$ lysate $(2 \mathrm{e} / \mu \mathrm{L})$ \\
\hline control & $\begin{array}{l}50 \mu \mathrm{L} \text { lysate }+1 \mu \mathrm{L} \mathrm{ddH} \mathrm{d}_{2} \mathrm{O} \\
(2 \mathrm{e} / \mu \mathrm{L})\end{array}$ \\
\hline RNase \& RNase inhibitor & $\begin{array}{l}1 \mu \mathrm{L}(0.1 \mu \mathrm{g}) \text { RNase; } \\
1 \mu \mathrm{L}(4 \mathrm{O} \mathrm{U}) \mathrm{RNase} \text { inhibitor; } \\
+8 \mu \mathrm{L} \text { buffer }(50 \mathrm{mM} \text { Tris } \mathrm{pH} 7.5,75 \mathrm{mM} \mathrm{NaCl}) \\
+40 \mu \mathrm{L} \text { lysate }(1.6 \mathrm{e} / \mu \mathrm{L})\end{array}$ \\
\hline RNase & $\begin{array}{l}50 \mu \mathrm{L} \text { lysate+ } \\
1 \mu \mathrm{L}(0.1 \mu \mathrm{g}) \mathrm{RNase} \\
(2 \mathrm{e} / \mu \mathrm{L})\end{array}$ \\
\hline
\end{tabular}


The western blot analysing the samples is shown in figure 36. The samples were stained for Slam and SHMT (control). It verifies, that RNase seems to have a destabilising effect on Slam. The RNase treated sample is fainter than the control sample and the RNase inhibitor reduces this effect. The band is stronger. SHMT is vastly unaffected by the RNase treatment. The samples vary in strength in a normal range. The RNase treated sample rather appears to be the strongest of the 4 bands.

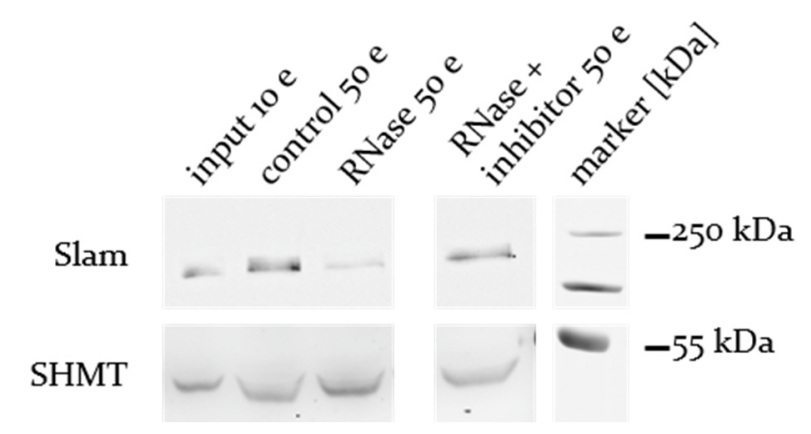

Figure 36 Degradation of Slam in lysate is faster in the presence of additional RNase. Western blot analysis of the experimental samples ascertaining the effect of additional RNase on Slam protein. The RNase-treated (RNase) sample shows less Slam after 10 min incubation time than the non-treated (control) and inhibitor-treated (RNase + inhibitor) sample. SHMT approximate quantity appears unaffected by the different treatments. The bands stained for SHMT show similar strengths. 


\section{Discussion}

\subsection{Purification of recombinant Slam}

The C-terminus of Slam appears to be involved in downstream signalling. It is for this reason and because of its profound predicted structure, which makes it the most promising part of Slam for crystallization attempts, that in this study purification strategies were mainly elaborated for the far C-terminus of Slam. The purification of Slam is intricate. Heterologous Slam does not display high solubility or stability. Further developing a purification strategy for C-terminal Slam was a balancing act between solubility and purity. For further purification attempts it could be advantageous to employ insect cells for expression and production of Slam or Slam truncations (Altmann et al. 1999). As it was reported in this study, Slams stability seems to be at least partially dependent on the presence of its RNA. Since there are no reasons to assume that Slam is an RNA-binding protein, it is likely part of a complex. This complex may stabilize Slam and has a much higher chance to also assemble, when in a biochemical surrounding that is similar to the evolving blastoderm. Drosophila S2 cells would be an example. In S2 cells Slam localizes to the membranes and binding partners are more likely present than in E. coli. Furthermore, known binding partners can be co-expressed to improve Slams stability and solubility during purification attempts.

In respect of the difficulties that come along with the solubilization and purification of Slam and Slam truncations and the chances of gaining good diffracting crystals from purified Slam truncations (taking its intrinsic disorder into account) it may be advisable to focus on functional experiments rather than working on structural objectives in the future. Bioinformatic analyses are a well-developed part of biochemical research and it is valid to put trust in the results in cases where a structure is unattainable.

\subsection{Biochemistry of Slam}

Slam and its mRNA colocalize and are part of the same RNP complex. The nature of this complex is not yet resolved. In this study is was shown, that Slam protein degradation in wild-type lysate accelerates in the presence of additional RNase. This suggests that the stability of the RNP is dependent on Slam mRNA or another RNA. Slams (predicted) missing structure likely makes it prone to degradation without any interactors. If the mRNA of Slam is an essential part of a complex that assembles around Slam it may protect it from early degradation. The elucidation of this complex would be an interesting subject to further studies. 
Wild-type Slam runs in a double band and displays a much higher weight than calculated $(+\sim 30 \mathrm{kDa})$ during SDS-PAGES. Further, Slam is phosphorylated. This was seen in mass spectrometric analyses during this thesis (data not shown). To what degree, is not clear. Serine, threonine, and tyrosine make up nearly $20 \%$ of Slams amino acids $(12,2 \%, 6,1 \%$ and $1,6 \%$, respectively). There is high potential for great phosphorylation. A phosphatase assay during this study supported the idea of Slam multiple phosphorylation. Upon phosphatase treatment the lower band deepened, which indicates a higher phosphorylation status of the upper band. Usually phosphorylation of a protein serves regulatory purposes. Interactors can or are hindered to bind upon phosphorylation of the target protein. Since Slam is likely an adaptor protein that attracts effectors to the FC (RhoGEF2, Patj, MyoII), a regulatory phosphorylation fits the concept.

The quantitative profile during first hours of Slam development exposed a pattern of the Slam double bands. The lower band (putatively less or dephosphorylated) clearly increased in strength approximately 2-3 hours after fertilization. This suggests a function of the lower band Slam or less dephosphorylated Slam during cellularization. After the time course of cellularization both bands again adopt similar strengths. Slam may be in a somewhat inactive state till start of cellularization and gets activated when it reaches the furrow to attract downstream effectors. It is also possible, however, that it is the inactivated variant of Slam since the lower band can also indicate the end of cellularization. A deeper look into this context is necessary to make final conclusions. First, antibodies against e.g. phosphoserines could be used to verify the phosphorylation of Slam during a western blot. This would also clarify if the lower band is less or dephosphorylated. Since the ubiquitin ligase Herc2 was coprecipitated with Slam it should also be looked for a possible ubiquitination of Slam. Secondly, embryos treated with immunofluorescent antibodies with subsequent microscopic analysis could shed light on spatial distribution of phosphorylated or non-phosphorylated (or ubiquitinylated) Slam in the fly before and during cellularization. Once the understanding of Slam PTMs has increased more subtle assays could be developed to understand its regulation during cellularization.

\subsection{Interactors of Slam}

Co-IP/mass spectrometric results of this thesis extended the knowledge of the Slam interactome. Several binding partners were suggested, some are likely to interact directly. Verification of direct or indirect interaction may be addressed in further studies. 
Slam regulates Rho1 and its effector Diaphanous by the direct biochemical interaction and recruitment of Rho1 activating RhoGEF2 to the furrow canal (Grosshans et al. 2005; Wenzl et al. 2010). As a result, Slam essentially influences cytoskeletal organization during cellularization. RhoGEF2 was not found as a binder of Slam during co-IP experiments conducted in the context of this thesis. But MyoII (zipper), one other effector of the Rho1-Rok signalling, was coprecipitated with Slam. The Rho1-Rok-MyoII pathway leads to actomyosin contraction. Slam is needed to localize MyoII to the FC and will also recruit it ectopically. There is a possibility that the recruitment of MyoII is through the upstream regulator RhoGEF2 but since it was not enriched (but identified) in the co-IP experiments it may not be involved. In my estimation Slam serves as an adapter protein for the Rho1-Rok pathway, which is indicated by the recruitment of both RhoGEF2 (other studies) and MyoII and several other actomyosin related proteins. In fact, the vast majority of the interacting proteins identified are actin related. These include Cindr as a possible direct interactor (through a $\mathrm{SH}_{3}$ binding motif on Slam), MyoV, MyoVIIA, the Arp2/3 complex and capping proteins. These results are also consistent with a yeast-two-hybrid-screen conducted earlier, where the formin Spire, Myoioa and formin-like (CG32138) were identified as Slam interactors (Laupsien 2012). Even though Actin was found (slightly) enriched in 3 of the 6 IP/Ripa- buffer samples, the low enrichment and the overall results contradict a direct contact of Slam to Actin filaments. The enrichment was very low (FE 4, 1.3 and 1.1) and in 2 of the 6 samples in was measured higher in the wild type sample (FE o.8 and 0.7 ) at comparably high spectral counting (SC 181 and 247, which is representative for all the measurements except IP buffer sample $C$, where Actin was not detected). In conclusion, the results of this thesis indicate that Slam is rather an actin-binding protein interactor than a direct actin-binder. Although it may not be a direct binder of actin filaments its impact on the actomyosin network during cellularization is immense. Without Slam the FC does not invaginate and cellularization cannot be completed. The interactors that were identified in this thesis support Slams role as an actomyosin regulator in the form of an adaptor promoting the assembly of proteins that lead to actomyosin-driven membrane invagination.

\section{Slam as adaptor}

The task of an adaptor protein is experimentally hard to distinguish from anchoring, scaffolding or docking functions. Terms and functions are overlapping and, to some degree, interchangeable. A simple definition is that adaptor proteins "facilitate signal transduction through interactions with other proteins" (Borowicz et al. 2020; Langeberg and Scott 2015). Such a function was shown for Slam through the necessity for the recruitment and the verification of direct binding of RhoGEF2, a regulator of the Rho-Rok pathway (Wenzl et al. 2010). The 
superordinate or adaptor role of Slam during this pathway is concreted with MyoII, another effector of the Rho-Rok signalling, being an interactor of Slam as shown by co-IP as a result of this thesis. Furthermore, the slam phenotype hints towards an assembling role during Rho-Rok signalling.

According to Borowicz et al. an adaptor protein should correspond to some characteristics: Slam is membrane associated but no transmembrane protein, which qualifies it for the title 'adaptor protein'. To our current knowledge Slam has no enzymatic activity and is not involved enzymatically in cellular signalling (Langeberg and Scott 2015). The protein should have no DNA- or RNA-binding activity. Slam does not display any known DNA- or RNA-binding domain and the binding of its mRNA seems to be facilitated by an associated, not yet identified, RNAbinder. "Adaptor proteins, which engage in the formation of stable complexes, are excluded" (Borowicz et al. 2020). Slams function during Drosophila development follows a strong spatiotemporal regulation, which suggest a flexibility in interaction and function. Binding partners identified in this study hint towards an involvement in several sub-processes during the course cellularization. RhoGEF2 (which was identified in a different study) and MyoII are part of the Rho-Rok pathway. Identified actin binding proteins like the Arp2/3 complex or capping proteins may be associated to Slams adaptor function in this pathway. Patj for example is involved in adherens junctions stabilisation, and its function is at least not directly associated to the Rho-Rok pathway. Cindr, Restin homolog and Jaguar are interactors, that indicate functions of Slam apart from Rho-Rok signalling. Under semi-harsh conditions most of the interactions are not retained, which suggests rather moderate and repetitive, or higher-order interactions. The difficulty of explaining the slam phenotype with one or two binding partners in a simple context is another hint towards a multi-protein binder with transient interactions and various functions during cellularization. Taken together, Slam fulfils all the requirements for a proper adaptor protein during a most sensibly regulated process of Drosophila development.

Adaptor proteins facilitate the right localization of their binders and to fulfil that task they need certain qualities (Borowicz et al. 2020; Langeberg and Scott 2015): binding domains, binding motifs and structural flexibility. A PDZ-binding motif and an $\mathrm{SH}_{3}$-binding motif have been identified for the recruitment of (at least) RhoGEF2 (PDZ), Patj (PDZ) and Cindr ( $\mathrm{SH}_{3}$ ).

Slam has no known domains and several results indicate little structure. The high intrinsic disorder suggests a great structural flexibility of Slam protein, a feature which is highly relevant for adaptors. This is (among other things) because they can easily be post-translationally modified, the disorder enables flexible links between structured domain and binding sites, it 
makes dynamic structure changes possible, it increases the hydrodynamic radius of the protein and promotes oligomerization (Borowicz et al. 2020; Iakoucheva 2004; Houtman et al. 2006; Hofmann et al. 2012; Cortese, Uversky, and Keith Dunker 2008).

There is a graphic in Borowiczs anniversary article, that I would like to present in this thesis. This is because, when I look at the models depicted (figure 37), that present the functions of adaptor proteins, I clearly recognize Slam. All the things we know about Slam point towards an adaptor function. Slam and its interactors define space and time for the membrane invagination during cellular blastoderm development. The Rho-Rok pathway with Dia and MyoII being two of its effectors, leads to actomyosin contraction and membrane invagination (Crawford et al. 1998). The localization of RhoGEF2, an upstream regulator of Rho1, and MyoII, an effector of Rhor signalling, both depend on Slam. The localization of both at the same place increases the probability of interaction of intermediate proteins. Bottleneck, an essential timer of dorsal closure was also found interacting with Slam during co-IP. For several interactors identified in this study further experiments have to be conducted to make final conclusions and interconnect the different functions of Slam during cellularization. I am positive, that following experiments will endorse an adaptor function of Slam.

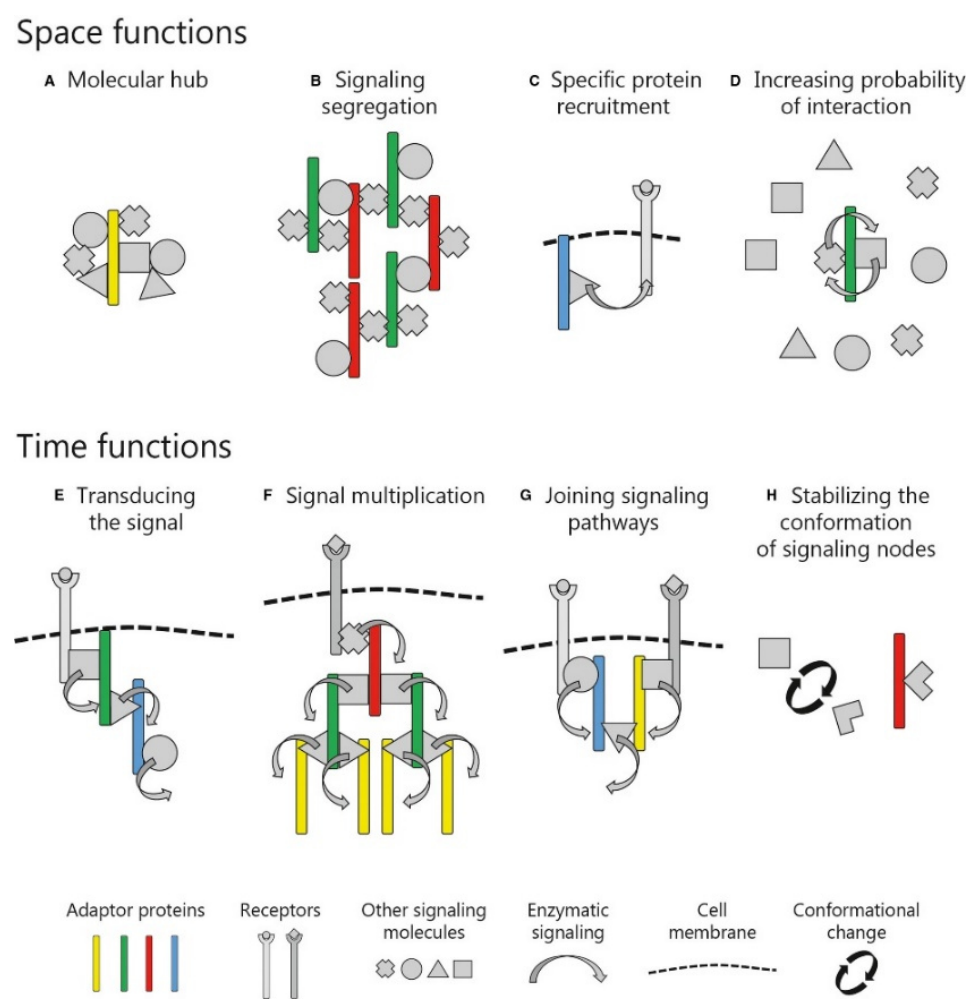

Figure 37 "Schematic overview of hypothetical models presenting functions of adaptor proteins", picture adapted from (Borowicz et al. 2020). Scientific results concerning Slam all point towards a possible adaptor function during Drosophila cellularization. Slam fulfils all the requirements for a proper adaptor and likely displays multiple spacial and temporal functions of the ones named above. 


\section{Localization of slam mRNA}

Yet unresolved is how Slam and its mRNA are recruited to and hold at the FC. We know that Rabil and the recycling endosome is essential for the localization of Slam and that Slam protein itself is required for the localization of its mRNA. No known RNA-binder was found enriched with Slam except for IGF-II in RIPA- buffer sample A-C. This enrichment was so low (FE 2,3; 1,4 and 1,3 respectively), that I will not discuss IGF-II as a Slam binding partner. It shall not stay unmentioned either. The process of Slam protein localization and accumulation at the FC takes only around $5 \mathrm{~min}$ (Yan et al. 2017), whereas Slams role in membrane invagination takes place in a timeframe of $45 \mathrm{~min}$ (Acharya et al. 2014). There is a higher chance of finding Slam binders, that are involved in the second process. To specifically find interactors participating in Slam and slam mRNA localization an embryo collection of narrower timed staging would be advised.

\subsection{Conclusion}

Several interactors of Slam were considered in this thesis. Patj was confirmed as one interactor of Slam. RhoGEF2 could not be found during this co-IP mass spectrometric approach. Cindr was detected as reliable as Patj and Slam displays a Cindr binding consensus sequence. I propose that Cindr is a direct interactor of Slam and should be investigated during cellularization as distinct protein and as Slam interactor. Restin homolog, Jaguar and MyoII are also good candidates for a direct interaction with Slam. These results should be verified by western blotting after a direct binding assay.

What may be considered, however, is that the vast majority of the proteins are interconnected to one another. Several interactions between detected proteins are reported (fig. 38 ). It is likely that not all the identified coimmunoprecipitated proteins are direct interactors of Slam and that some have pulled down others.

I propose that Slam functions as an adaptor protein for the Rho-Rok pathway and other proteins, that have been discussed in this thesis and need to be subject of further investigations, which is regulated by phosphorylation (and possibly ubiquitinylation) during the course of cellularization.

It is part of a complex that contains its own mRNA, but no obvious RNA-binding protein was detected among the coimmunoprecipitated proteins. Interactors involved in Slam translational control and the complex in which Slam is associated with its RNA make up only a small fraction of Slam protein and only at the onset of cellularization. In this IP - mass spectrometric approach, the Slam RNP complex likely fell under the detection limit. The experiment has to be optimized 
for the isolation of the Slam RNP. The mediator between Slam, possible other components of the RNP and slam mRNA has yet to be identified.

For further characterization of the nature of interactions it is advised to perform an APcrosslinking experiment to distinguish between primary/secondary and tertiary binders. The expansion of our knowledge concerning the interactive network Slam is part of, and which is delicately regulated during embryogenesis in flies as well as in other organisms will advance our understanding of the developmental processes Slam and its interactors are involved with.

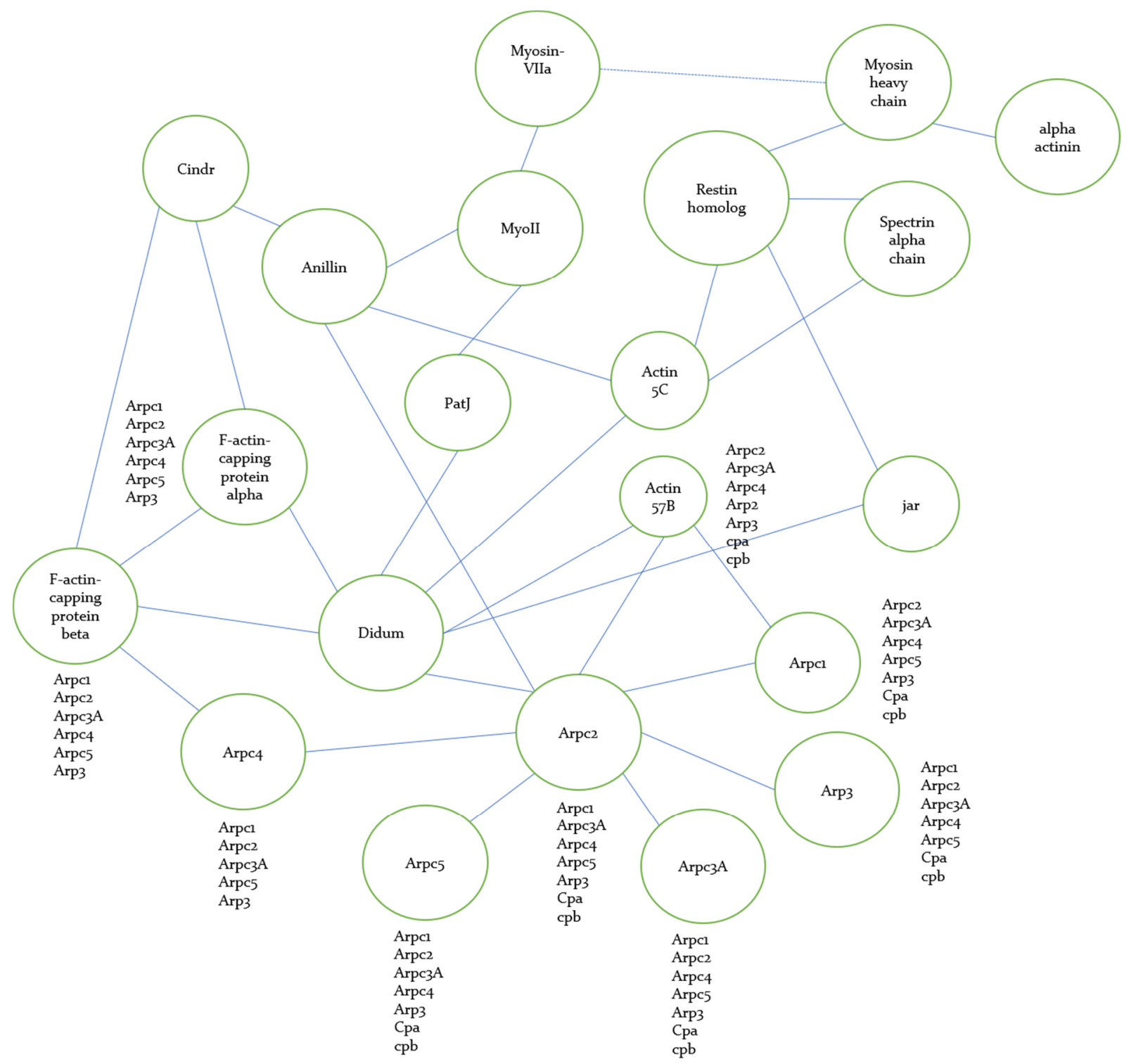

Figure 38 Interactions between a selection of identified proteins coprecipitated with Slam referred to flybase.org (physical interactions). Interactors that did not fit into the depiction were listed under the respective protein. 


\section{Appendix}

Table 10 Normalized total spectrum counts of enriched proteins. UniProt reference numbers are indicated. GFPSlam served as bait, wild type (WT) as control. FE fold enrichment, $p(B H)$ Benjamini-Hochberg corrected p-value, nd non-detectable

IP buffer sample A

\begin{tabular}{|c|c|c|c|c|c|c|c|}
\hline protein & UniProt ID & GFPSlam & $\begin{array}{l}\text { GFPSlam } \\
\text { (tech. replicate) }\end{array}$ & WT & $\begin{array}{l}\text { WT } \\
\text { (tech. replicate) }\end{array}$ & & $\mathrm{BH})$ \\
\hline Slow as molasses (Slam) & $\mathrm{Q}_{9} \mathrm{VME}_{5}$ & 991 & $1 \quad 1025$ & nd & nd & & 0.00028 \\
\hline PatJ & $\mathrm{A}_{4} \mathrm{~V}_{1} \mathrm{~B}_{2}$ & 215 & 230 & nd & nd & & o.oo1 \\
\hline Restin homolog & $\mathrm{Q}_{9} \mathrm{VJE}_{5}$ & 31 & 24 & nd & nd & & 0.019 \\
\hline Herc2 & Q9VR91 & 240 & 261 & 3 & 8 & 46 & 0.0021 \\
\hline Neuralized E3 ubiquitin protein ligase 4 & AoAıL4AAD6 & 49 & 67 & 6 & 5 & 11 & 0.029 \\
\hline Cindr & $\mathrm{Q}_{9} \mathrm{VA}_{3} 6$ & 39 & 36 & nd & nd & & 0.00076 \\
\hline Bottleneck & $\mathrm{P}_{40794}$ & 17 & 16 & 1 & o & 33 & 0.0021 \\
\hline $\operatorname{Actin}\left({ }_{5} \mathrm{C} / 57 \mathrm{~B} / 57 \mathrm{~B}\right)$ & $\mathrm{X}_{2} \mathrm{JCP} 8 / \mathrm{P}_{53501}$ & 440 & 442 & 122 & 127 & 4 & $<0.00010$ \\
\hline Alpha Spectrin & M9PGV6 & 37 & 36 & 1 & 2 & 24 & 0.00058 \\
\hline Alpha actinin & FoJAG6 & 47 & 48 & 5 & 4 & 11 & 0.00026 \\
\hline F-actin-capping protein subunit alpha & $\mathrm{Q}_{9} \mathrm{~W}_{2} \mathrm{No}_{0}$ & 27 & 24 & 2 & 4 & 9 & 0.0026 \\
\hline F-actin-capping protein subunit beta & $\mathrm{P}_{4} 8603$ & 37 & 36 & 4 & 4 & 9 & 0.0068 \\
\hline Arpc1 & $\mathrm{O}_{97182}$ & 10 & 11 & 2 & 2 & 5 & 0.0055 \\
\hline Arpc2 & $\mathrm{Q}_{9} \mathrm{VIM}_{5}$ & 23 & 27 & 4 & 2 & 8 & o.o11 \\
\hline Arpc4 & $\mathrm{Q}_{9} \mathrm{VMH}_{2}$ & 9 & 8 & nd & nd & & 0.0015 \\
\hline Arpc5 & Q9VQD8 & 13 & 13 & 1 & 1 & 13 & 0.00095 \\
\hline Arp2 & $\mathrm{P}_{45} 888$ & 7 & 6 & nd & nd & & 0.0035 \\
\hline Arp3 & $\mathrm{P}_{32392}$ & 20 & 19 & 1 & 2 & 13 & 0.0026 \\
\hline Kinesin light chain & $\mathrm{P}_{4} 6824$ & 16 & 14 & 7 & 5 & 3 & 0.017 \\
\hline Jaguar & $\mathrm{AoAoB}_{4} \mathrm{KGX}_{1}$ & 257 & 288 & 7 & 4 & 50 & 0.0035 \\
\hline Dilute class unconventional myosin & $\mathrm{AlZ6Z}_{9}$ & 133 & 151 & nd & nd & & 0.0040 \\
\hline Myosin-VIIa & $\mathrm{Q}_{9} \mathrm{~V}_{3} \mathrm{Z6}$ & 33 & 21 & 1 & 2 & 18 & 0.056 \\
\hline Myosin $\mathrm{II}^{1}$ & Q99323 (B4J9L6) & 174 & 200 & 18 & 19 & 10 & 0.0058 \\
\hline
\end{tabular}

Table 11 Normalized total spectrum counts of proteins that have been enriched in sample A (IP). Same proteins have been checked in this sample for comparison. UniProt reference numbers are indicated. GFPSlam served as bait, wild type (WT) as control. FE fold enrichment, $p(B H)$ Benjamini-Hochberg corrected p-value, nd non-detectable

IP buffer sample B

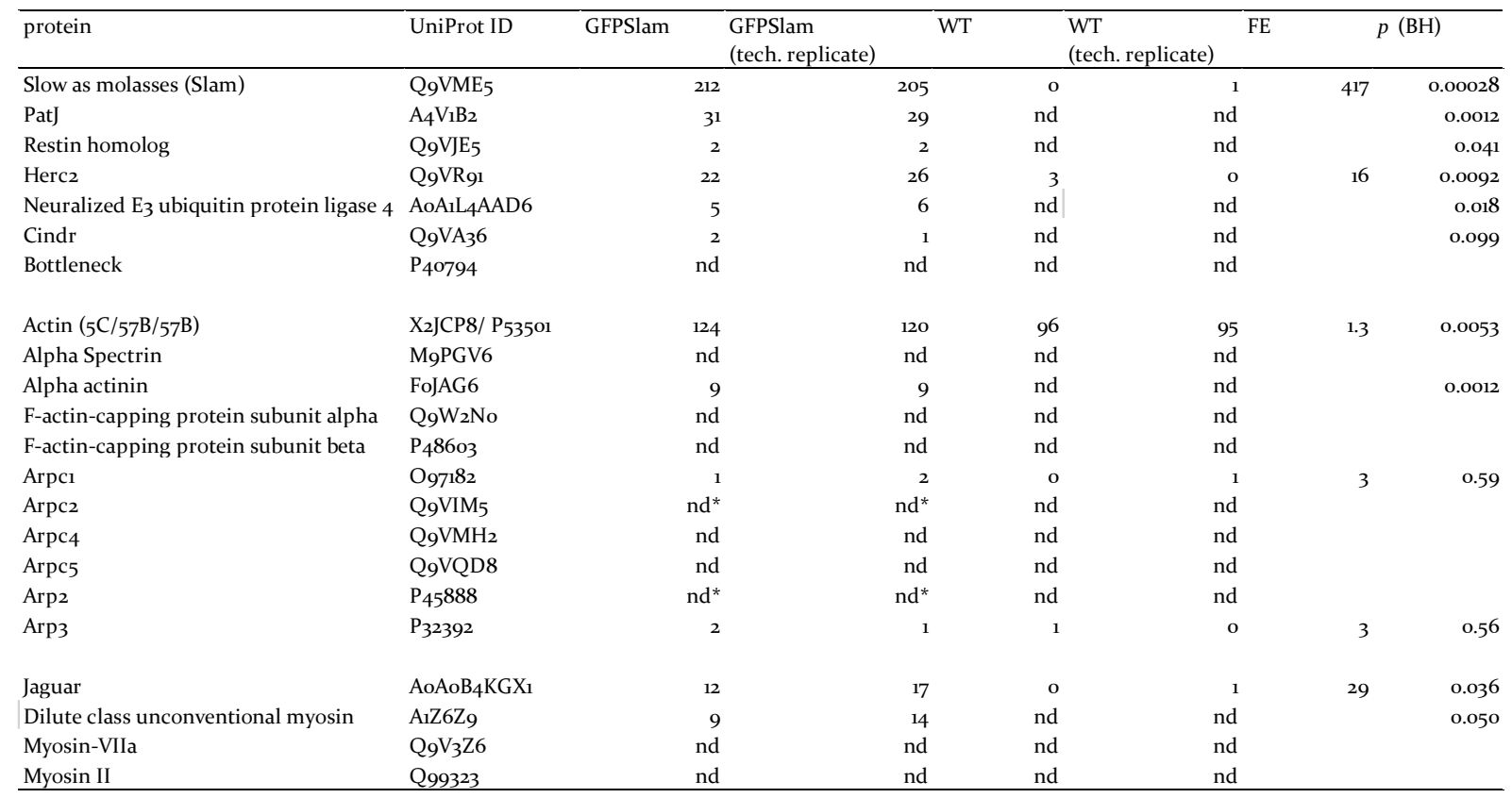


Table 12 Normalized total spectrum counts of proteins that have been enriched in sample A (IP). Same proteins have been checked in this sample for comparison. UniProt reference numbers are indicated. GFPSlam served as bait, wild type (WT) as control. FE fold enrichment, $p(B H)$ Benjamini-Hochberg corrected p-value, nd non-detectable

IP buffer sample C

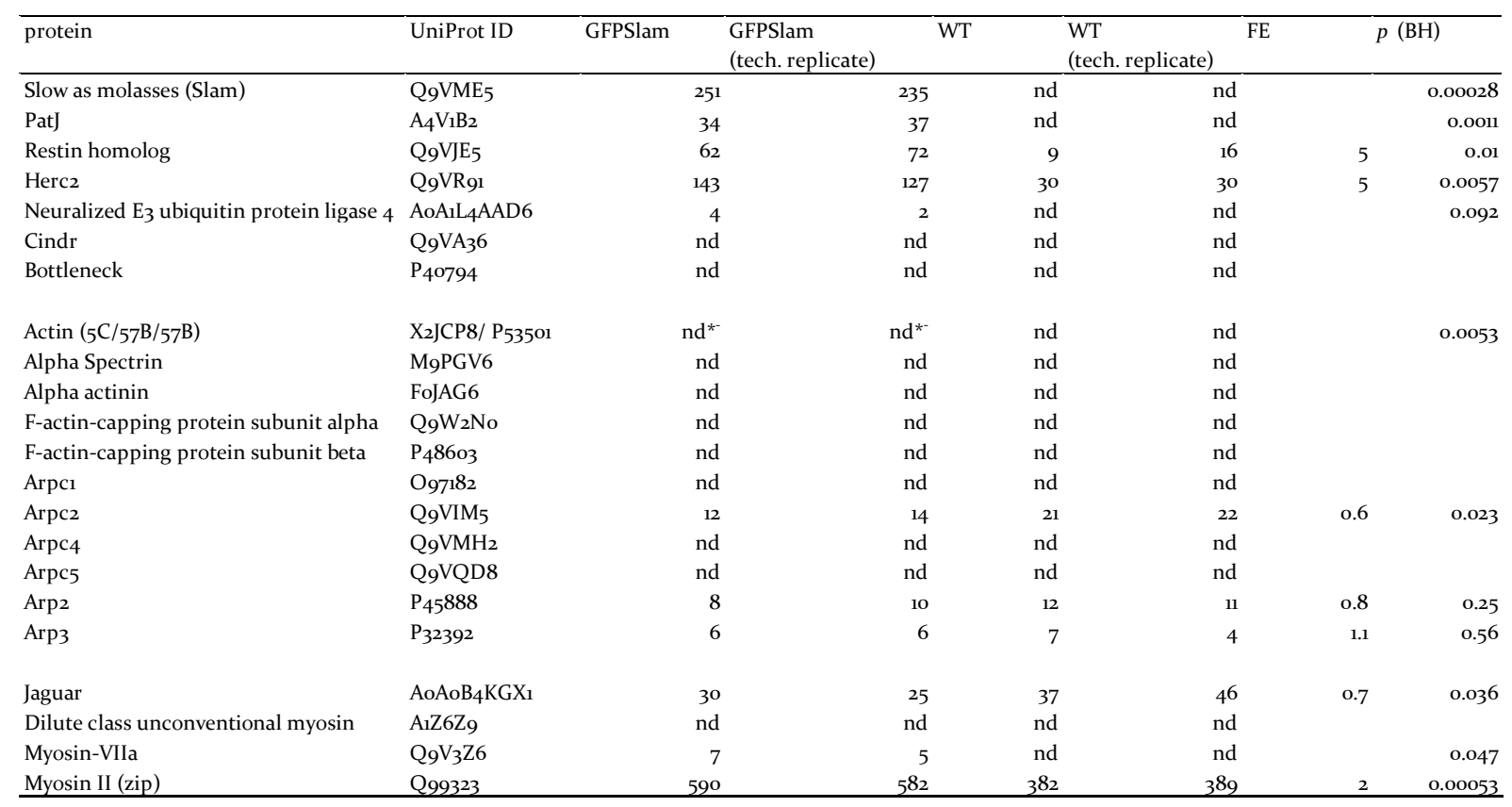

Table 13 Normalized total spectrum counts of proteins that have been enriched in sample A (IP). Same proteins have been checked in this sample for comparison. UniProt reference numbers are indicated. GFPSlam served as bait, wild type (WT) as control. FE fold enrichment, $p(B H)$ Benjamini-Hochberg corrected p-value (ANOVA), nd non-detectable

RIPA(-) buffer sample A

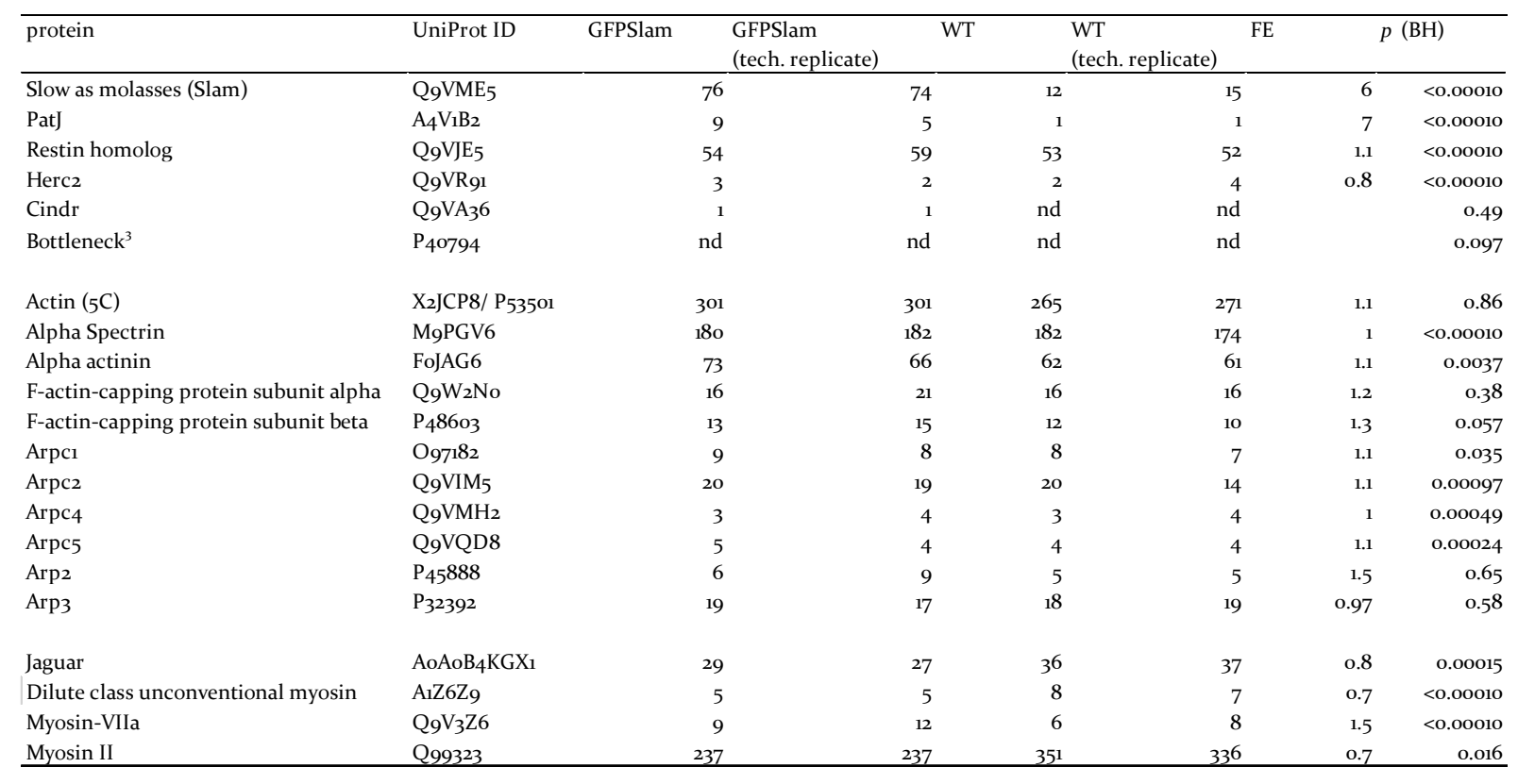


Table 14 Normalized total spectrum counts of proteins that have been enriched in sample A (IP). Same proteins have been checked in this sample for comparison. UniProt reference numbers are indicated. GFPSlam served as bait, wild type (WT) as control. FE fold enrichment, $p(B H)$ Benjamini-Hochberg corrected p-value (ANOVA), nd non-detectable

RIPA(-) buffer sample B

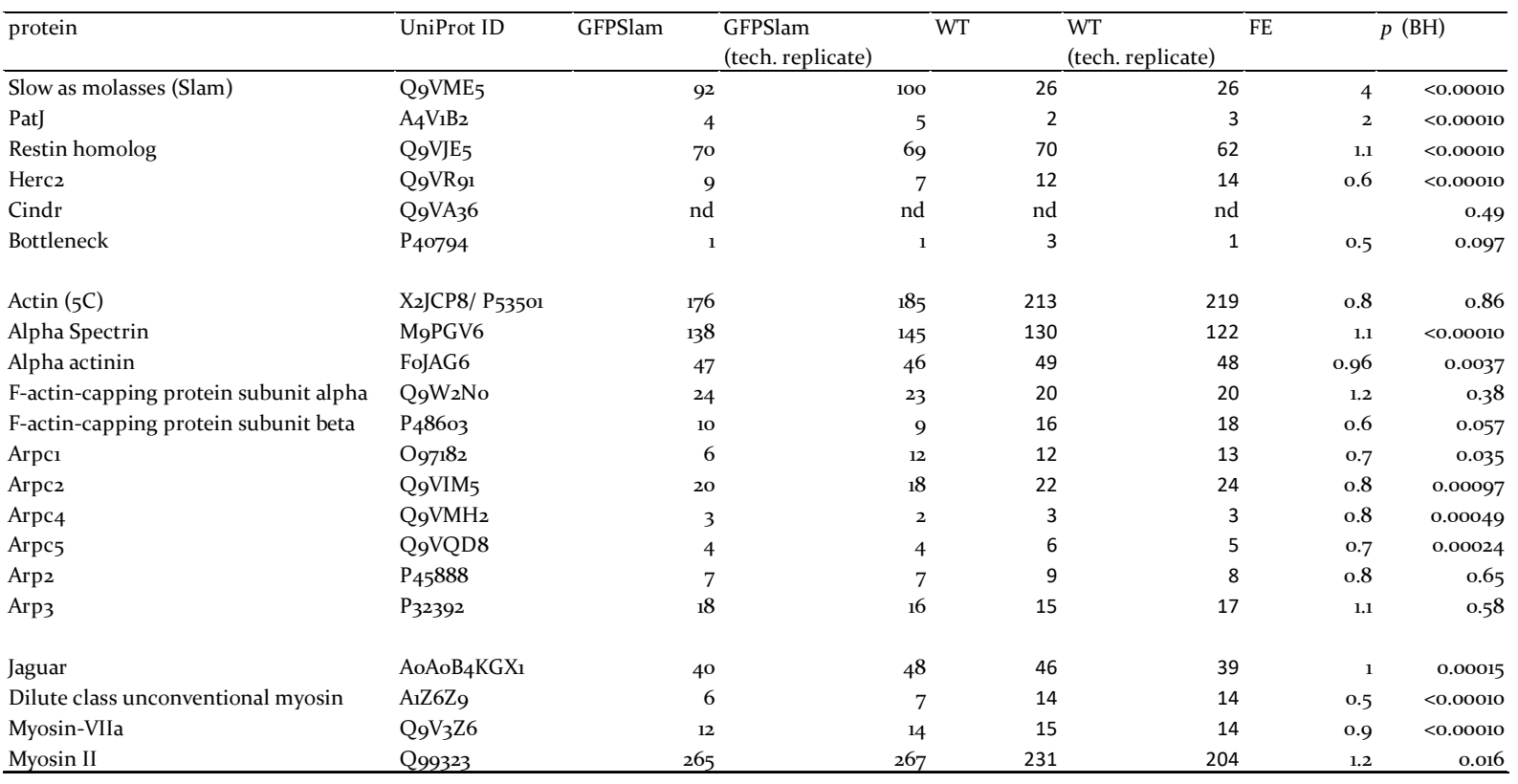

Table 15 Normalized total spectrum counts of proteins that have been enriched in sample A (IP). Same proteins have been checked in this sample for comparison. UniProt reference numbers are indicated. GFPSlam served as bait, wild type (WT) as control. FE fold enrichment, $p(B H)$ Benjamini-Hochberg corrected p-value (ANOVA), nd non-detectable

RIPA(-) buffer sample C

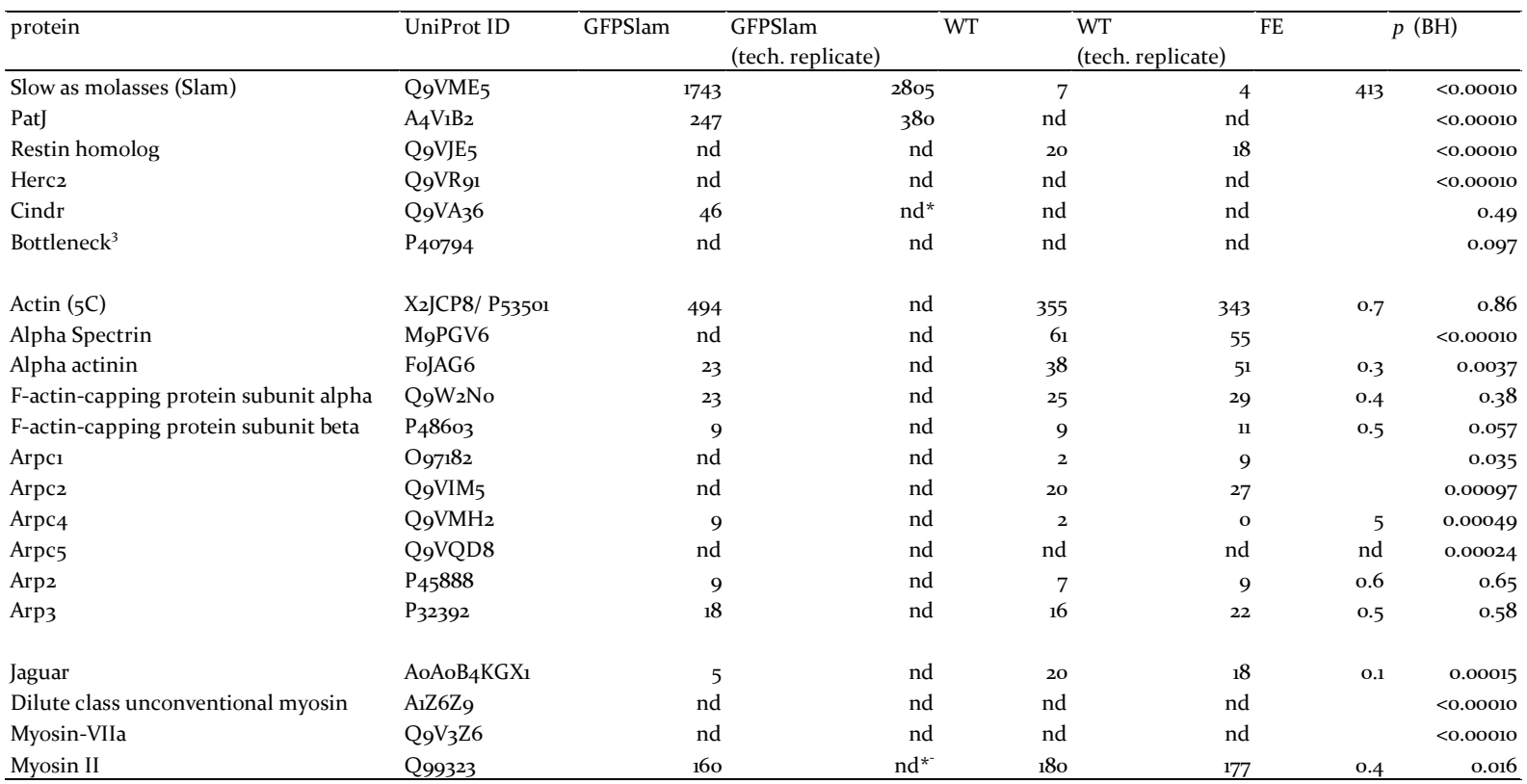

*non-detectable under given settings but detectable and enriched under changed settings (protein threshold 1.o \% FDR; min. peptides: 2; and peptide

*-non-detectable under given settings but detectable and NOT enriched under above-mentioned settings

${ }^{1}$ not assigned to DROME but other Drosophila subspecies 


\section{References}

Acharya, Sreemukta. 2014. 'Localisation and Function of Slam in the Early Drosophila Embryo'. Göttingen: Universität Göttingen.

Acharya, Sreemukta, Philip Laupsien, Christian Wenzl, Shuling Yan, and Jörg Großhans. 2014. 'Function and Dynamics of Slam in Furrow Formation in Early Drosophila Embryo'. Developmental Biology 386 (2): 371-84. https://doi.org/10.1016/j.ydbio.2013.12.022.

Afshar, K., B. Stuart, and S. A. Wasserman. 2000. 'Functional Analysis of the Drosophila Diaphanous FH Protein in Early Embryonic Development'. Development (Cambridge, England) 127 (9): 1887-97.

Al-Hakim, Abdallah K., Mikhail Bashkurov, Anne-Claude Gingras, Daniel Durocher, and Laurence Pelletier. 2012. 'Interaction Proteomics Identify NEURL4 and the HECT E3 Ligase HERC 2 as Novel Modulators of Centrosome Architecture'. Molecular \& Cellular Proteomics 11 (6): M111.014233. https://doi.org/10.1074/mcp.M111.014233.

Altmann, Friedrich, Erika Staudacher, Iain B. H. Wilson, and Leopold März. 1999. 'Insect Cells as Hosts for the Expression of Recombinant Glycoproteins'. In Glycotechnology, edited by E. G. Berger, H. Clausen, and R. D. Cummings, 29-43. Boston, MA: Springer US. https://doi.org/10.1007/978-1-4615-5257-4_3.

Ardito, Fatima, Michele Giuliani, Donatella Perrone, Giuseppe Troiano, and Lorenzo Lo Muzio. 2017. 'The Crucial Role of Protein Phosphorylation in Cell Signaling and Its Use as Targeted Therapy (Review)'. International Journal of Molecular Medicine 40 (2): 271-80. https://doi.org/10.3892/ijmm.2017.3036.

Barrett, K., M. Leptin, and J. Settleman. 1997. 'The Rho GTPase and a Putative RhoGEF Mediate a Signaling Pathway for the Cell Shape Changes in Drosophila Gastrulation'. Cell 91 (7): 905-15. https://doi.org/10.1016/soo92-8674(oo)80482-1.

Bartel, David P. 2018. 'Metazoan MicroRNAs'. Cell 173 (1): 20-51. https://doi.org/10.1016/j.cell.2018.03.0o6.

Bate, Michael, and Alfonso Martinez Arias, eds. 1993. The Development of Drosophila Melanogaster. Plainview, NY: Cold Spring Harbor Laboratory Press.

Batschkus, Sarah, Goekhan Cingoez, Henning Urlaub, Nicolai Miosge, Christian Kirschneck, Philipp Meyer-Marcotty, and Christof Lenz. 2018. 'A New Albumin-Depletion Strategy Improves Proteomic Research of Gingival Crevicular Fluid from Periodontitis Patients'. Clinical Oral Investigations 22 (3): 1375-84. https://doi.org/10.1007/s00784-017-2213-o.

Beaven, Robin, Nikola S. Dzhindzhev, Yue Qu, Ines Hahn, Federico Dajas-Bailador, Hiroyuki Ohkura, and Andreas Prokop. 2015. 'Drosophila CLIP-19o and Mammalian CLIP-170 Display Reduced Microtubule plus End Association in the Nervous System'. Edited by Erika Holzbaur. Molecular Biology of the Cell 26 (8): 1491-1508. https://doi.org/10.1091/mbc.E14-06-1083.

Beronja, Slobodan, and Ulrich Tepass. 2002. 'Cellular Morphogenesis'. Developmental Cell 2 (4): 382-84. https://doi.org/10.1016/S1534-5807(02)oo16o-o.

Blake-Hedges, Caitlyn, and Timothy L. Megraw. 2019. 'Coordination of Embryogenesis by the Centrosome in Drosophila Melanogaster'. In The Golgi Apparatus and Centriole, edited by Malgorzata Kloc, 67:277-321. Results and Problems in Cell Differentiation. Cham: Springer International Publishing. https://doi.org/10.1007/978-3-030-23173-6_12.

Borowicz, Paweł, Hanna Chan, Anette Hauge, and Anne Spurkland. 2020. 'Adaptor Proteins: Flexible and Dynamic Modulators of Immune Cell Signalling'. Scandinavian Journal of Immunology 92 (5): e12951. https://doi.org/10.1111/sji.12951.

Brandt, Annely, Fani Papagiannouli, Nicole Wagner, Michaela Wilsch-Bräuninger, Martina Braun, Eileen E. Furlong, Silke Loserth, et al. 2006. 'Developmental Control of Nuclear Size and Shape by Kugelkern and Kurzkern'. Current Biology 16 (6): 543-52. https://doi.org/10.1016/j.cub.2006.01.051.

Bray, Sarah J. 2006. 'Notch Signalling: A Simple Pathway Becomes Complex'. Nature Reviews Molecular Cell Biology 7 (9): 678-89. https://doi.org/10.1038/nrm2oo9. 
Bruck, Serawit, Tobias B. Huber, Robert J. Ingham, Kyoungtae Kim, Hanspeter Niederstrasser, Paul M. Allen, Tony Pawson, John A. Cooper, and Andrey S. Shaw. 20o6. 'Identification of a Novel Inhibitory Actin-Capping Protein Binding Motif in CD2-Associated Protein'. The Journal of Biological Chemistry 281 (28): 19196-203. https://doi.org/10.1074/jbc.M6oo166200.

Buchan, Daniel W A, and David T Jones. 2019. 'The PSIPRED Protein Analysis Workbench: 20 Years On'. Nucleic Acids Research 47 (W1): W402-7. https://doi.org/10.1093/nar/gkz297.

Campos-Ortega, Jose A., and Volker Hartenstein. 2014. The Embryonic Development of Drosophila Melanogaster. 2. ed., Softcover reprint of the hardcover 2. ed. 1997. Berlin [u.a.] Springer.

Cormont, Mireille, Isidoro Metón, Muriel Mari, Pascale Monzo, Frédérique Keslair, Chantell Gaskin, Timothy E. McGraw, and Yannick Le Marchand-Brustel. 2003. 'CD2AP/CMS Regulates Endosome Morphology and Traffic to the Degradative Pathway through Its Interaction with Rab4 and c-Cbl'. Traffic (Copenhagen, Denmark) 4 (2): 97-112. https://doi.org/10.1034/j.1600-0854.2003.40205.x.

Cortese, Marc S., Vladimir N. Uversky, and A. Keith Dunker. 20o8. 'Intrinsic Disorder in Scaffold Proteins: Getting More from Less'. Progress in Biophysics and Molecular Biology 98 (1): 85-106. https://doi.org/10.1016/j.pbiomolbio.2008.05.007.

Cramer, Louise P. 200o. 'Myosin VI'. Journal of Cell Biology 150 (6): F121-26. https://doi.org/10.1083/jcb.150.6.F121.

Crawford, Janice M, Nicholas Harden, Thomas Leung, Louis Lim, and Daniel P Kiehart. 1998. 'Cellularization InDrosophila MelanogasterIs Disrupted by the Inhibition of Rho Activity and the Activation of Cdc42 Function'. Developmental Biology 204 (1): 151-64. https://doi.org/10.1006/dbio.1998.9061.

D’Avino, Pier Paolo, Tetsuya Takeda, Luisa Capalbo, Wei Zhang, Kathryn S. Lilley, Ernest D. Laue, and David M. Glover. 2008. 'Interaction between Anillin and RacGAP5oC Connects the Actomyosin Contractile Ring with Spindle Microtubules at the Cell Division Site'. Journal of Cell Science 121 (Pt 8): 1151-58. https://doi.org/10.1242/jcs.026716.

De Simone, A., C. Kitchen, A. H. Kwan, M. Sunde, C. M. Dobson, and D. Frenkel. 2012. 'Intrinsic Disorder Modulates Protein Self-Assembly and Aggregation'. Proceedings of the National Academy of Sciences 109 (18): 6951-56. https://doi.org/10.1073/pnas.1118048109.

Desnos, C., S. Huet, I. Fanget, C. Chapuis, C. Bottiger, V. Racine, J.-B. Sibarita, J.-P. Henry, and F. Darchen. 2007. 'Myosin Va Mediates Docking of Secretory Granules at the Plasma Membrane'. Journal of Neuroscience 27 (39): 10636-45. https://doi.org/10.1523/JNEUROSCI.1228-07.2007.

Dikic, I. 2003. 'Mechanisms Controlling EGF Receptor Endocytosis and Degradation'. Biochemical Society Transactions 31 (Pt 6): 1178-81. https://doi.org/10.1042/bsto311178.

Falo-Sanjuan, Julia, and Sarah J. Bray. 2021. 'Membrane Architecture and Adherens Junctions Contribute to Strong Notch Pathway Activation'. Preprint. Developmental Biology. https://doi.org/10.1101/2021.05.26.445755.

Falo-Sanjuan, Julia, Nicholas C. Lammers, Hernan G. Garcia, and Sarah J. Bray. 2019. 'Enhancer Priming Enables Fast and Sustained Transcriptional Responses to Notch Signaling'. Developmental Cell 50 (4): 411-425.e8. https://doi.org/10.1016/j.devcel.2019.07.002.

Ferrandon, Dominique, Lisa Elphick, Christiane Nüsslein-Volhard, and Daniel St Johnston. 1994. 'Staufen Protein Associates with the 3 'UTR of Bicoid MRNA to Form Particles That Move in a Microtubule-Dependent Manner'. Cell 79 (7): 1221-32. https://doi.org/10.1016/0092-8674(94)90013-2.

Field, C. M., and B. M. Alberts. 1995. 'Anillin, a Contractile Ring Protein That Cycles from the Nucleus to the Cell Cortex'. The Journal of Cell Biology 131 (1): 165-78. https://doi.org/10.1083/jcb.131.1.165. 
Field, Christine M., Margaret Coughlin, Steve Doberstein, Thomas Marty, and William Sullivan. 2005. 'Characterization of Anillin Mutants Reveals Essential Roles in Septin Localization and Plasma Membrane Integrity'. Development 132 (12): 2849-6o. https://doi.org/10.1242/dev.01843.

Figard, Lauren, Heng Xu, Hernan G. Garcia, Ido Golding, and Anna Marie Sokac. 2013. 'The Plasma Membrane Flattens Out to Fuel Cell-Surface Growth during Drosophila Cellularization'. Developmental Cell 27 (6): 648-55. https://doi.org/10.1016/j.devcel.2013.11.0o6.

Finan, D., M. A. Hartman, and J. A. Spudich. 2011. 'Proteomics Approach to Study the Functions of Drosophila Myosin VI through Identification of Multiple Cargo-Binding Proteins'. Proceedings of the National Academy of Sciences 108 (14): 5566-71. https://doi.org/10.1073/pnas.1101415108.

Foe, V. E., and B. M. Alberts. 1983. 'Studies of Nuclear and Cytoplasmic Behaviour during the Five Mitotic Cycles That Precede Gastrulation in Drosophila Embryogenesis'. Journal of Cell Science 61 (May): 31-70.

Foe, V. E., C. M. Field, and G. M. Odell. 200o. 'Microtubules and Mitotic Cycle Phase Modulate Spatiotemporal Distributions of F-Actin and Myosin II in Drosophila Syncytial Blastoderm Embryos'. Development (Cambridge, England) 127 (9): 1767-87.

Fullilove, Susan L., and Antone G. Jacobson. 1971. 'Nuclear Elongation and Cytokinesis in Drosophila Montana'. Developmental Biology 26 (4): 560-77. https://doi.org/10.1016/0012-1606(71)90141-2.

Gaidos, Gabriel, Shefali Soni, Duane J. Oswald, Paul A. Toselli, and Kathrin H. Kirsch. 2007. 'Structure and Function Analysis of the CMS/CIN85 Protein Family Identifies ActinBundling Properties and Heterotypic-Complex Formation'. Journal of Cell Science 120 (Pt 14): 2366-77. https://doi.org/10.1242/jcs.004333.

Gasteiger, Elisabeth, Christine Hoogland, Alexandre Gattiker, S'everine Duvaud, Marc R. Wilkins, Ron D. Appel, and Amos Bairoch. 2005. 'Protein Identification and Analysis Tools on the ExPASy Server'. In The Proteomics Protocols Handbook, edited by John M. Walker, 571-607. Totowa, NJ: Humana Press. https://doi.org/10.1385/1-59259-890-0:571.

Grosshans, Jörg, Christian Wenzl, Hans-Martin Herz, Slawomir Bartoszewski, Frank Schnorrer, Nina Vogt, Heinz Schwarz, and H.-Arno Müller. 2005. 'RhoGEF2 and the Formin Dia Control the Formation of the Furrow Canal by Directed Actin Assembly during Drosophila Cellularisation'. Development (Cambridge, England) 132 (5): 1009-20. https://doi.org/10.1242/dev.01669.

Guruharsha, K. G., Jean-François Rual, Bo Zhai, Julian Mintseris, Pujita Vaidya, Namita Vaidya, Chapman Beekman, et al. 2011. 'A Protein Complex Network of Drosophila Melanogaster'. Cell 147 (3): 690-703. https://doi.org/10.1016/j.cell.2011.08.047.

Haglund, Kaisa, Ioannis P. Nezis, Dafne Lemus, Caroline Grabbe, Jørgen Wesche, Knut Liestøl, Ivan Dikic, Ruth Palmer, and Harald Stenmark. 2010. 'Cindr Interacts with Anillin to Control Cytokinesis in Drosophila Melanogaster'. Current Biology 20 (10): 944-50. https://doi.org/10.1016/j.cub.2010.03.068.

Halstead, J. M., J. H. Wilbertz, F. Wippich, T. Lionnet, A. Ephrussi, and J. A. Chao. 2016. 'TRICK: A Single-Molecule Method for Imaging the First Round of Translation in Living Cells and Animals'. Methods in Enzymology 572: 123-57. https://doi.org/10.1016/bs.mie.2016.02.027.

Halstead, James M., Timothée Lionnet, Johannes H. Wilbertz, Frank Wippich, Anne Ephrussi, Robert H. Singer, and Jeffrey A. Chao. 2015. 'Translation. An RNA Biosensor for Imaging the First Round of Translation from Single Cells to Living Animals'. Science (New York, N.Y.) 347 (6228): 1367-1671. https://doi.org/10.1126/science.aaa3380.

Hasson, T, and M S Mooseker. 1994. 'Porcine Myosin-VI: Characterization of a New Mammalian Unconventional Myosin.' Journal of Cell Biology 127 (2): 425-40. https://doi.org/10.1083/jcb.127.2.425. 
Hasson, Tama, Peter G. Gillespie, Jesus A. Garcia, Richard B. MacDonald, Yi-dong Zhao, Ann G. Yee, Mark S. Mooseker, and David P. Corey. 1997. 'Unconventional Myosins in InnerEar Sensory Epithelia'. Journal of Cell Biology 137 (6): 1287-1307. https://doi.org/10.1083/jcb.137.6.1287.

He, Bing, Adam Martin, and Eric Wieschaus. 2016. 'Flow-Dependent Myosin Recruitment during Drosophila Cellularization Requires Zygotic Dunk Activity'. Development (Cambridge, England) 143 (13): 2417-30. https://doi.org/10.1242/dev.131334.

He, Fahu, Kohei Saito, Naohiro Kobayashi, Takushi Harada, Satoru Watanabe, Takanori Kigawa, Peter Güntert, et al. 2009. 'Structural and Functional Characterization of the NHR1 Domain of the Drosophila Neuralized E3 Ligase in the Notch Signaling Pathway'. Journal of Molecular Biology 393 (2): 478-95. https://doi.org/10.1016/j.jmb.2009.08.020.

Heintzelman, M. B., T. Hasson, and M. S. Mooseker. 1994. 'Multiple Unconventional Myosin Domains of the Intestinal Brush Border Cytoskeleton'. Journal of Cell Science 107 ( Pt 12) (December): 3535-43.

Hicks, Jennifer L., Wu-Min Deng, Aaron D. Rogat, Kathryn G. Miller, and Mary Bownes. 1999. 'Class VI Unconventional Myosin Is Required for Spermatogenesis in Drosophila'.

Edited by Paul T. Matsudaira. Molecular Biology of the Cell 10 (12): 4341-53. https://doi.org/10.1091/mbc.10.12.4341.

Hofmann, H., A. Soranno, A. Borgia, K. Gast, D. Nettels, and B. Schuler. 2012. 'Polymer Scaling Laws of Unfolded and Intrinsically Disordered Proteins Quantified with SingleMolecule Spectroscopy'. Proceedings of the National Academy of Sciences 109 (40): 16155-6o. https://doi.org/10.1073/pnas.1207719109.

Houtman, Jon C D, Hiroshi Yamaguchi, Mira Barda-Saad, Alex Braiman, Brent Bowden, Ettore Appella, Peter Schuck, and Lawrence E Samelson. 2006. 'Oligomerization of Signaling Complexes by the Multipoint Binding of GRB2 to Both LAT and SOS1'. Nature Structural \& Molecular Biology 13 (9): 798-805. https://doi.org/10.1038/nsmb1133.

H. S. Lu, Candy, Kai Liu, Lay Pheng Tan, and Shao Q. Yao. 2012. 'Current Chemical Biology Tools for Studying Protein Phosphorylation and Dephosphorylation'. Chemistry - A European Journal 18 (1): 28-39. https://doi.org/10.1002/chem.201103206.

Hunter, Christine, Patricia Sung, Eyal D. Schejter, and Eric Wieschaus. 2002. 'Conserved Domains of the Nullo Protein Required for Cell-Surface Localization and Formation of Adherens Junctions'. Edited by Judith Kimble. Molecular Biology of the Cell 13 (1): 14657. https://doi.org/10.1091/mbc.01-08-0418.

Hunter, Christine, and Eric Wieschaus. 200o. 'Regulated Expression of Nullo Is Required for the Formation of Distinct Apical and Basal Adherens Junctions in the Drosophila Blastoderm'. Journal of Cell Biology 150 (2): 391-402. https://doi.org/10.1083/jcb.150.2.391.

Hurd, Thomas Ryan, Beate Herrmann, Julia Sauerwald, Justina Sanny, Markus Grosch, and Ruth Lehmann. 2016. 'Long Oskar Controls Mitochondrial Inheritance in Drosophila Melanogaster'. Developmental Cell 39 (5): 560-71. https://doi.org/10.1016/j.devcel.2016.11.004.

Hutchings, Nicholas J., Nicholas Clarkson, Robert Chalkley, A. Neil Barclay, and Marion H. Brown. 2003. 'Linking the T Cell Surface Protein CD2 to the Actin-Capping Protein CAPZ via CMS and CIN85'. The Journal of Biological Chemistry 278 (25): 22396-403. https://doi.org/10.1074/jbc.M302540200.

Iakoucheva, L. M. 2004. 'The Importance of Intrinsic Disorder for Protein Phosphorylation'. Nucleic Acids Research 32 (3): 1037-49. https://doi.org/10.1093/nar/gkh253.

Ibnsouda, S., F. Schweisguth, G. de Billy, and A. Vincent. 1993. 'Relationship between Expression of Serendipity Alpha and Cellularisation of the Drosophila Embryo as Revealed by Interspecific Transformation'. Development (Cambridge, England) 119 (2): $471-83$.

Ibnsouda, Saad, François Schweisguth, Denis Jullien, Claudia Kücherer, Jean-Antoine Lepesant, and Alain Vincent. 1995. 'Evolutionarily Conserved Positive and Negative Cis-Acting 
Elements Control the Blastoderm-Specific Expression of the Drosophila Serendipity $\alpha$ Cellularisation Gene'. Mechanisms of Development 49 (1-2): 71-82.

https://doi.org/10.1016/0925-4773(94)00304-6.

Imai, Yuzuru, Yoshito Kobayashi, Tsuyoshi Inoshita, Hongrui Meng, Taku Arano, Kengo Uemura, Takeshi Asano, et al. 2015. 'The Parkinson's Disease-Associated Protein Kinase LRRK2 Modulates Notch Signaling through the Endosomal Pathway'. Edited by KahLeong Lim. PLOS Genetics 11 (9): e1005503. https://doi.org/10.1371/journal.pgen.1005503.

Ji, Huan-Hong, Hai-Man Zhang, Mei Shen, Lin-Lin Yao, and Xiang-dong Li. 2015. 'The Motor Function of Drosophila Melanogaster Myosin- 5 Is Activated by Calcium and CargoBinding Protein DRabi1'. Biochemical Journal 469 (1): 135-44. https://doi.org/10.1042/BJ20141330.

Johnson, Ruth I., Midori J. Seppa, and Ross L. Cagan. 2008. 'The Drosophila CD2AP/CIN85 Orthologue Cindr Regulates Junctions and Cytoskeleton Dynamics during Tissue Patterning'. The Journal of Cell Biology 180 (6): 1191-1204. https://doi.org/10.1083/jcb.200706108.

Jones, David T. 1999. 'Protein Secondary Structure Prediction Based on Position-Specific Scoring Matrices 1 1Edited by G. Von Heijne'. Journal of Molecular Biology 292 (2): 195202. https://doi.org/10.1006/jmbi.1999.3091.

Jones, David T., and Domenico Cozzetto. 2015. 'DISOPRED3: Precise Disordered Region Predictions with Annotated Protein-Binding Activity'. Bioinformatics 31 (6): 857-63. https://doi.org/10.1093/bioinformatics/btu744.

Jones, Jennifer, and Paul M. Macdonald. 2015. 'Neurl4 Contributes to Germ Cell Formation and Integrity in Drosophila'. Biology Open 4 (8): 937-46. https://doi.org/10.1242/bio.o12351.

Karess, Roger E., Xiao-jia Chang, Kevin A. Edwards, Sanjay Kulkarni, Isabel Aguilera, and Daniel P. Kiehart. 1991. 'The Regulatory Light Chain of Nonmuscle Myosin Is Encoded by Spaghetti-Squash, a Gene Required for Cytokinesis in Drosophila'. Cell 65 (7): 117789. https://doi.org/10.1016/oo92-8674(91)90013-O.

Kiehart, D. P. 1990. 'The Actin Membrane Skeleton in Drosophila Development'. Seminars in Cell Biology 1 (5): 325-39.

Kiehart, Daniel P. 1990. 'Molecular Genetic Dissection of Myosin Heavy Chain Function'. Cell 6o (3): 347-50. https://doi.org/10.1016/oo92-8674(90)90583-Z.

Kiehart, Daniel P, Josef D Franke, Mark K Chee, R A Montague, Tung-ling Chen, John Roote, and Michael Ashburner. 2004. 'Drosophila Crinkled, Mutations of Which Disrupt Morphogenesis and Cause Lethality, Encodes Fly Myosin VIIA'. Genetics 168 (3): 133752. https://doi.org/10.1534/genetics.104.026369.

Kowanetz, Katarzyna, Iwona Szymkiewicz, Kaisa Haglund, Marcin Kowanetz, Koraljka Husnjak, Jonathan D. Taylor, Philippe Soubeyran, Ulla Engstrom, John E. Ladbury, and Ivan Dikic. 2003. 'Identification of a Novel Proline-Arginine Motif Involved in CIN85Dependent Clustering of $\mathrm{Cbl}$ and down-Regulation of Epidermal Growth Factor Receptors'. The Journal of Biological Chemistry 278 (41): 39735-46. https://doi.org/10.1074/jbc.M304541200.

Kowanetz, Katarzyna, Janos Terzic, and Ivan Dikic. 2003. 'Dab2 Links CIN85 with ClathrinMediated Receptor Internalization'. FEBS Letters 554 (1-2): 81-87. https://doi.org/10.1016/soo14-5793(o3)o1111-6.

Krauss, Jana, Sonia López de Quinto, Christiane Nüsslein-Volhard, and Anne Ephrussi. 2009. 'Myosin-V Regulates Oskar MRNA Localization in the Drosophila Oocyte'. Current Biology 19 (12): 1058-63. https://doi.org/10.1016/j.cub.2009.04.062.

Kurakin, Alexei V., Susan Wu, and Dale E. Bredesen. 2003. 'Atypical Recognition Consensus of CIN85/SETA/Ruk SH3 Domains Revealed by Target-Assisted Iterative Screening'. The Journal of Biological Chemistry 278 (36): 34102-9. https://doi.org/10.1074/jbc.M305264200.

Laganà, Alessandro, Dario Veneziano, Francesco Russo, Alfredo Pulvirenti, Rosalba Giugno, Carlo Maria Croce, and Alfredo Ferro. 2015. 'Computational Design of Artificial RNA 
Molecules for Gene Regulation'. Methods in Molecular Biology (Clifton, N.J.) 1269: 393412. https://doi.org/10.1007/978-1-4939-2291-8_25.

Langeberg, Lorene K., and John D. Scott. 2015. 'Signalling Scaffolds and Local Organization of Cellular Behaviour'. Nature Reviews Molecular Cell Biology 16 (4): 232-44. https://doi.org/10.1038/nrm3966.

Lantz, Valerie A., and Kathryn G. Miller. 1998. 'A Class VI Unconventional Myosin Is Associated with a Homologue of a Microtubule-Binding Protein, Cytoplasmic Linker Protein-170, in Neurons and at the Posterior Pole of Drosophila Embryos'. Journal of Cell Biology 140 (4): 897-910. https://doi.org/10.1083/jcb.140.4.897.

Laupsien, Philip. 2012. 'Die Funktion von Slow-as-Molasses (Slam) Während Der Zellularisierung von Drosophila Melanogaster'. Heidelberg: University of Heidelberg.

Lecuit, T., and E. Wieschaus. 200o. 'Polarized Insertion of New Membrane from a Cytoplasmic Reservoir during Cleavage of the Drosophila Embryo'. The Journal of Cell Biology 150 (4): 849-6o. https://doi.org/10.1083/jcb.150.4.849.

Lecuit, Thomas. 2004. 'Junctions and Vesicular Trafficking during Drosophila Cellularization'. Journal of Cell Science 117 (Pt 16): 3427-33. https://doi.org/10.1242/jcs.01312.

Lecuit, Thomas, Reba Samanta, and Eric Wieschaus. 2002. 'Slam Encodes a Developmental Regulator of Polarized Membrane Growth during Cleavage of the Drosophila Embryo'. Developmental Cell 2 (4): 425-36. https://doi.org/10.1016/S1534-5807(02)oo141-7.

Lehtonen, Sanna, Eero Lehtonen, Krystyna Kudlicka, Harry Holthöfer, and Marilyn G. Farquhar. 2004. 'Nephrin Forms a Complex with Adherens Junction Proteins and CASK in Podocytes and in Madin-Darby Canine Kidney Cells Expressing Nephrin'. The American Journal of Pathology 165 (3): 923-36. https://doi.org/10.1016/Sooo29440(10)63354-8.

Lehtonen, Sanna, Fang Zhao, and Eero Lehtonen. 2002. 'CD2-Associated Protein Directly Interacts with the Actin Cytoskeleton'. American Journal of Physiology. Renal Physiology 283 (4): F734-743. https://doi.org/10.1152/ajprenal.o0312.2001.

Li, Ji, Sehyun Kim, Tetsuo Kobayashi, Feng-Xia Liang, Nina Korzeniewski, Stefan Duensing, and Brian D Dynlacht. 2012. 'Neurl4, a Novel Daughter Centriole Protein, Prevents Formation of Ectopic Microtubule Organizing Centres'. EMBO Reports 13 (6): 547-53. https://doi.org/10.1038/embor.2012.40.

Li, X., M. Wilmanns, J. Thornton, and M. Kohn. 2013. 'Elucidating Human PhosphataseSubstrate Networks'. Science Signaling 6 (275): rs10-rs1o. https://doi.org/10.1126/scisignal.2003203.

Lundgren, Deborah H, Sun-Il Hwang, Linfeng Wu, and David K Han. 2010. 'Role of Spectral Counting in Quantitative Proteomics'. Expert Review of Proteomics 7 (1): 39-53. https://doi.org/10.1586/epr.09.69.

Lye, Claire M., Huw W. Naylor, and Bénédicte Sanson. 2014. 'Subcellular Localisations of the CPTI Collection of YFP-Tagged Proteins in Drosophila Embryos'. Development 141 (20): 4006-17. https://doi.org/10.1242/dev.111310.

Mahowald, Anthony P. 1963. 'Ultrastructural Differentiations during Formation of the Blastoderm in the Drosophila Melanogaster Embryo'. Developmental Biology 8 (2): 186204. https://doi.org/10.1016/oo12-1606(63)90041-1.

Mazumdar, Aveek, and Manjari Mazumdar. 2002. 'How One Becomes Many: Blastoderm Cellularization InDrosophila Melanogaster'. BioEssays 24 (11): 1012-22. https://doi.org/10.1002/bies.10184.

Mermall, V., and K. G. Miller. 1995. 'The 95F Unconventional Myosin Is Required for Proper Organization of the Drosophila Syncytial Blastoderm'. The Journal of Cell Biology 129 (6): 1575-88. https://doi.org/10.1083/jcb.129.6.1575.

Mermall, Valerie, James G. McNally, and Kathryn G. Miller. 1994. 'Transport of Cytoplasmic Particles Catalysed by an Unconventional Myosin in Living Drosophila Embryos'. Nature 369 (6481): 56o-62. https://doi.org/10.1038/369560ao. 
Merrill, P. T., D. Sweeton, and E. Wieschaus. 1988. 'Requirements for Autosomal Gene Activity during Precellular Stages of Drosophila Melanogaster'. Development (Cambridge, England) 104 (3): 495-509.

Miller, K G, and D P Kiehart. 1995. 'Fly Division.' Journal of Cell Biology 131 (1): 1-5. https://doi.org/10.1083/jcb.131.1.1.

Monzo, Pascale, Nils C. Gauthier, Frédérique Keslair, Agnès Loubat, Christine M. Field, Yannick Le Marchand-Brustel, and Mireille Cormont. 2005. 'Clues to CD2-Associated Protein Involvement in Cytokinesis'. Molecular Biology of the Cell 16 (6): 2891-2902. https://doi.org/10.1091/mbc.e04-09-0773.

Müller, H A, and E Wieschaus. 1996. 'Armadillo, Bazooka, and Stardust Are Critical for Early Stages in Formation of the Zonula Adherens and Maintenance of the Polarized Blastoderm Epithelium in Drosophila.' Journal of Cell Biology 134 (1): 149-63. https://doi.org/10.1083/jcb.134.1.149.

Mustonen, Harri, Anna Lepistö, Sanna Lehtonen, Eero Lehtonen, Pauli Puolakkainen, and Eero Kivilaakso. 2005. 'CD2AP Contributes to Cell Migration and Adhesion in Cultured Gastric Epithelium'. Biochemical and Biophysical Research Communications 332 (2): 426-32. https://doi.org/10.1016/j.bbrc.2005.04.140.

Petritsch, Claudia, Gaia Tavosanis, Christoph W. Turck, Lily Y. Jan, and Yuh Nung Jan. 2003. 'The Drosophila Myosin VI Jaguar Is Required for Basal Protein Targeting and Correct Spindle Orientation in Mitotic Neuroblasts'. Developmental Cell 4 (2): 273-81. https://doi.org/10.1016/S1534-5807(03)00020-0.

Pocha, Shirin Meher, Anna Shevchenko, and Elisabeth Knust. 2011. 'Crumbs Regulates Rhodopsin Transport by Interacting with and Stabilizing Myosin V'. Journal of Cell Biology 195 (5): 827-38. https://doi.org/10.1083/jcb.201105144.

Postner, M. A., and E. F. Wieschaus. 1994. 'The Nullo Protein Is a Component of the ActinMyosin Network That Mediates Cellularization in Drosophila Melanogaster Embryos'. Journal of Cell Science 107 ( Pt 7) (July): 1863-73.

Provance, D. W., M. Wei, V. Ipe, and J. A. Mercer. 1996. 'Cultured Melanocytes from Dilute Mutant Mice Exhibit Dendritic Morphology and Altered Melanosome Distribution'. Proceedings of the National Academy of Sciences 93 (25): 14554-58. https://doi.org/10.1073/pnas.93.25.14554.

Raff, J. W., and D. M. Glover. 1989. 'Centrosomes, and Not Nuclei, Initiate Pole Cell Formation in Drosophila Embryos'. Cell 57 (4): 611-19. https://doi.org/10.1016/oo92-8674(89)90130$\mathrm{x}$.

Ramos, Andres, Stefan Grünert, Jan Adams, David R. Micklem, Mark R. Proctor, Stefan Freund, Mark Bycroft, Daniel St Johnston, and Gabriele Varani. 200o. 'RNA Recognition by a Staufen Double-Stranded RNA-Binding Domain'. The EMBO Journal 19 (5): 997-1009. https://doi.org/10.1093/emboj/19.5.997.

Reversi, Alessandra, Eva Loeser, Devaraj Subramanian, Carsten Schultz, and Stefano De Renzis. 2014. 'Plasma Membrane Phosphoinositide Balance Regulates Cell Shape during Drosophila Embryo Morphogenesis'. Journal of Cell Biology 205 (3): 395-408. https://doi.org/10.1083/jcb.201309079.

Rickard, Janet E., and Thomas E. Kreis. 1996. 'CLIPs for Organellemicrotubule Interactions'. Trends in Cell Biology 6 (5): 178-83. https://doi.org/10.1016/o962-8924(96)10017-9.

Rikhy, Richa, Manos Mavrakis, and Jennifer Lippincott-Schwartz. 2015. 'Dynamin Regulates Metaphase Furrow Formation and Plasma Membrane Compartmentalization in the Syncytial Drosophila Embryo'. Biology Open 4 (3): 301-11. https://doi.org/10.1242/bio.20149936.

Robinson, R. C. 2001. 'Crystal Structure of Arp2/3 Complex'. Science 294 (5547): 1679-84. https://doi.org/10.1126/science.1066333.

Rose, L S, and E Wieschaus. 1992. 'The Drosophila Cellularization Gene Nullo Produces a Blastoderm-Specific Transcript Whose Levels Respond to the Nucleocytoplasmic Ratio.' Genes E Development 6 (7): 1255-68. https://doi.org/10.1101/gad.6.7.1255. 
Rost, Burkhard, and Chris Sander. 1993. 'Prediction of Protein Secondary Structure at Better than 70\% Accuracy'. Journal of Molecular Biology 232 (2): 584-99. https://doi.org/10.1006/jmbi.1993.1413.

Rothwell, W. F., P. Fogarty, C. M. Field, and W. Sullivan. 1998. 'Nuclear-Fallout, a Drosophila Protein That Cycles from the Cytoplasm to the Centrosomes, Regulates Cortical Microfilament Organization'. Development (Cambridge, England) 125 (7): 1295-1303.

Royou, Anne, Christine Field, John C. Sisson, William Sullivan, and Roger Karess. 2004. 'Reassessing the Role and Dynamics of Nonmuscle Myosin II during Furrow Formation in Early Drosophila Embryos'. Molecular Biology of the Cell 15 (2): 838-50. https://doi.org/10.1091/mbc.e03-06-0440.

Rubin, G. M. 200o. 'A Drosophila Complementary DNA Resource'. Science 287 (5461): 2222-24. https://doi.org/10.1126/science.287.5461.2222.

Sacco, Francesca, Livia Perfetto, Luisa Castagnoli, and Gianni Cesareni. 2012. 'The Human Phosphatase Interactome: An Intricate Family Portrait'. FEBS Letters 586 (17): 2732-39. https://doi.org/10.1016/j.febslet.2012.05.008.

Sambrook, Joseph, and David W. Russell. 2001. Molecular Cloning: A Laboratory Manual. 3rd ed. Cold Spring Harbor, N.Y: Cold Spring Harbor Laboratory Press.

Schejter, E. D., and E. Wieschaus. 1993. 'Bottleneck Acts as a Regulator of the Microfilament Network Governing Cellularization of the Drosophila Embryo'. Cell 75 (2): 373-85. https://doi.org/10.1016/oo92-8674(93)80078-s.

Schlessinger, A., G. Yachdav, and B. Rost. 2006. 'PROFbval: Predict Flexible and Rigid Residues in Proteins'. Bioinformatics 22 (7): 891-93. https://doi.org/10.1093/bioinformatics/btlo32.

Schlessinger, Avner, Marco Punta, Guy Yachdav, Laszlo Kajan, and Burkhard Rost. 2009. 'Improved Disorder Prediction by Combination of Orthogonal Approaches'. Edited by Joseph P. R. O. Orgel. PLoS ONE 4 (2): e4433. https://doi.org/10.1371/journal.pone.0oo4433.

Schmidt, Anja, Zhiyi Lv, and Jörg Großhans. 2018. 'ELMO and Sponge Specify Subapical Restriction of Canoe and Formation of the Subapical Domain in Early Drosophila Embryos'. Development 145 (2): dev157909. https://doi.org/10.1242/dev.157909.

Schweisguth, F, J A Lepesant, and A Vincent. 1990. 'The Serendipity Alpha Gene Encodes a Membrane-Associated Protein Required for the Cellularization of the Drosophila Embryo.' Genes \& Development 4 (6): 922-31. https://doi.org/10.1101/gad.4.6.922.

Sechi, Stefano, Anna Frappaolo, Roberta Fraschini, Luisa Capalbo, Marco Gottardo, Giorgio Belloni, David M. Glover, Alan Wainman, and Maria Grazia Giansanti. 2017. 'Rab1 Interacts with GOLPH3 and Controls Golgi Structure and Contractile Ring Constriction during Cytokinesis in Drosophila Melanogaster'. Open Biology 7 (1): 160257. https://doi.org/10.1098/rsob.160257.

Self, Tim, Tama Sobe, Neal G. Copeland, Nancy A. Jenkins, Karen B. Avraham, and Karen P. Steel. 1999. 'Role of Myosin VI in the Differentiation of Cochlear Hair Cells'. Developmental Biology 214 (2): 331-41. https://doi.org/10.1006/dbio.1999.9424.

Sen, Arnab, Zsanett Nagy-Zsvér-Vadas, and Michael P. Krahn. 2012. 'Drosophila PATJ Supports Adherens Junction Stability by Modulating Myosin Light Chain Activity'. Journal of Cell Biology 199 (4): 685-98. https://doi.org/10.1083/jcb.201206064.

Sharp, S. J., J. Schaack, L. Cooley, D. J. Burke, and D. Söll. 1985. 'Structure and Transcription of Eukaryotic TRNA Genes'. CRC Critical Reviews in Biochemistry 19 (2): 107-44. https://doi.org/10.3109/10409238509082541.

Simpson, L., and E. Wieschaus. 1990. 'Zygotic Activity of the Nullo Locus Is Required to Stabilize the Actin-Myosin Network during Cellularization in Drosophila'. Development (Cambridge, England) 110 (3): 851-63.

Sisson, John C., Christine Field, Richard Ventura, Anne Royou, and William Sullivan. 2000. 'Lava Lamp, a Novel Peripheral Golgi Protein, Is Required for Drosophila Melanogaster 
Cellularization'. Journal of Cell Biology 151 (4): 905-18.

https://doi.org/10.1083/jcb.151.4.905.

St Johnston, Daniel, Dirk Beuchle, and Christiane Nüsslein-Volhard. 1991. 'Staufen, a Gene Required to Localize Maternal RNAs in the Drosophila Egg'. Cell 66 (1): 51-63. https://doi.org/10.1016/oo92-8674(91)90138-O.

Stein, Jennifer A., Heather Tarczy Broihier, Lisa A. Moore, and Ruth Lehmann. 2002. 'Slow as Molasses Is Required for Polarized Membrane Growth and Germ Cell Migration in Drosophila'. Development 129 (16): 3925-34. https://doi.org/10.1242/dev.129.16.3925.

Stevenson, Victoria, Andrew Hudson, Lynn Cooley, and William E Theurkauf. 2002. 'Arp2/3Dependent Psuedocleavage Furrow Assembly in Syncytial Drosophila Embryos'. Current Biology 12 (9): 705-11. https://doi.org/10.1016/So960-9822(02)oo807-2.

Sullivan, W., P. Fogarty, and W. Theurkauf. 1993. 'Mutations Affecting the Cytoskeletal Organization of Syncytial Drosophila Embryos'. Development (Cambridge, England) 118 (4): 1245-54.

Thapar, Roopa. 2015. 'Structural Basis for Regulation of RNA-Binding Proteins by Phosphorylation'. ACS Chemical Biology 10 (3): 652-66. https://doi.org/10.1021/cb50086ox.

Theurkauf, W. E. 1994. 'Actin Cytoskeleton. Through the Bottleneck'. Current Biology: CB 4 (1): 76-78. https://doi.org/10.1016/so96o-9822(oo)ooo19-1.

Thomas, Jeffrey H., and Eric Wieschaus. 2004. 'Src64 and Tec29 Are Required for Microfilament Contraction during Drosophila Cellularization'. Development 131 (4): 863-71. https://doi.org/10.1242/dev.00989.

Tóth, Judit, Mihály Kovács, Fei Wang, László Nyitray, and James R. Sellers. 2005. 'Myosin V from Drosophila Reveals Diversity of Motor Mechanisms within the Myosin V Family'. Journal of Biological Chemistry 280 (34): 30594-603. https://doi.org/10.1074/jbc.M505209200.

Vicente-Manzanares, Miguel, Xuefei Ma, Robert S. Adelstein, and Alan Rick Horwitz. 2009. 'Non-Muscle Myosin II Takes Centre Stage in Cell Adhesion and Migration'. Nature Reviews Molecular Cell Biology 10 (11): 778-9o. https://doi.org/10.1038/nrm2786.

Welsch, T., N. Endlich, W. Kriz, and K. Endlich. 2001. 'CD2AP and P130Cas Localize to Different F-Actin Structures in Podocytes'. American Journal of Physiology. Renal Physiology 281 (4): F769-777. https://doi.org/10.1152/ajprenal.2001.281.4.F769.

Wenzl, Christian, Shuling Yan, Philip Laupsien, and Jörg Großhans. 2010. 'Localization of RhoGEF2 during Drosophila Cellularization Is Developmentally Controlled by Slam'. Mechanisms of Development 127 (7-8): 371-84. https://doi.org/10.1016/j.mod.2010.01.001.

Willmer, Pat. 1990. Invertebrate Relationships: Patterns in Animal Evolution. 1st ed. Cambridge University Press. https://doi.org/10.1017/CBO9780511623547.

Wilson, Gayle M., Andrew B. Fielding, Glenn C. Simon, Xinzi Yu, Paul D. Andrews, Rebecca S. Hames, Andrew M. Frey, Andrew A. Peden, Gwyn W. Gould, and Rytis Prekeris. 2005. 'The FIP3-Rabı Protein Complex Regulates Recycling Endosome Targeting to the Cleavage Furrow during Late Cytokinesis'. Molecular Biology of the Cell 16 (2): 849-6o. https://doi.org/10.1091/mbc.e04-10-0927.

Winkler, Franziska, Maria Kriebel, Michaela Clever, Stephanie Gröning, and Jörg Großhans. 2017. 'Essential Function of the Serine Hydroxymethyl Transferase (SHMT) Gene During Rapid Syncytial Cell Cycles in Drosophila'. G3 Genes|Genomes|Genetics 7 (7): 2305-14. https://doi.org/10.1534/g3.117.043133.

Woolner, Sarah, and William M. Bement. 2009. 'Unconventional Myosins Acting Unconventionally'. Trends in Cell Biology 19 (6): 245-52. https://doi.org/10.1016/j.tcb.2009.03.003.

$\mathrm{Wu}$, Xufeng, Blair Bowers, Kang Rao, Qin Wei, and John A. Hammer. 1998. 'Visualization of Melanosome Dynamics within Wild-Type and Dilute Melanocytes Suggests a Paradigm for Myosin V Function In Vivo'. Journal of Cell Biology 143 (7): 1899-1918. https://doi.org/10.1083/jcb.143.7.1899. 
Yamashita, R. A. 200o. 'Identification and Analysis of the Myosin Superfamily in Drosophila: A Database Approach'. Journal of Muscle Research and Cell Motility 21 (6): 491-505. https://doi.org/10.1023/A:1026589626422.

Yan, Shuling, Sreemukta Acharya, Stephanie Gröning, and Jörg Großhans. 2017. 'Slam Protein Dictates Subcellular Localization and Translation of Its Own MRNA'. Edited by Simon Bullock. PLOS Biology 15 (12): e2003315. https://doi.org/10.1371/journal.pbio.2003315.

Yan, Shuling, and Jörg Großhans. 2018. 'Localization and Translation Control of Slam in Drosophila Cellularization'. Fly 12 (3-4): 191-98. https://doi.org/10.1080/19336934.2018.1520574.

Yan, Shuling, Zhiyi Lv, Moritz Winterhoff, Christian Wenzl, Thomas Zobel, Jan Faix, Sven Bogdan, and Jörg Großhans. 2013. 'The F-BAR Protein Cip4/Toca-1 Antagonizes the Formin Diaphanous in Membrane Stabilization and Compartmentalization'. Journal of Cell Science, January, jcs.118422. https://doi.org/10.1242/jcs.118422.

Yang, Y., M. Kovacs, T. Sakamoto, F. Zhang, D. P. Kiehart, and J. R. Sellers. 20o6. 'Dimerized Drosophila Myosin VIIa: A Processive Motor'. Proceedings of the National Academy of Sciences 103 (15): 5746-51. https://doi.org/10.1073/pnas.0509935103.

Young, P. E., T. C. Pesacreta, and D. P. Kiehart. 1991. 'Dynamic Changes in the Distribution of Cytoplasmic Myosin during Drosophila Embryogenesis'. Development (Cambridge, England) 111 (1): 1-14.

Zallen, Jennifer A., Yehudit Cohen, Andrew M. Hudson, Lynn Cooley, Eric Wieschaus, and Eyal D. Schejter. 2002. 'SCAR Is a Primary Regulator of Arp2/3-Dependent Morphological Events in Drosophila'. The Journal of Cell Biology 156 (4): 689-701. https://doi.org/10.1083/jcb.200109057.

Zolakar, M, and I Erk. 1976. 'Division and Migration of Nuclei during Early Embryogenesis of Drosophila Melanogaster.' 97-106: 97-106. 